Pacific

Journal of

Mathematics

SPLITTINGS OF THE METAPLECTIC COVERS OF SOME REDUCTIVE DUAL PAIRS

\title{
SHU-Yen PAN
}

Volume 199 No. 1

May 2001 


\title{
SPLITTINGS OF THE METAPLECTIC COVERS OF SOME REDUCTIVE DUAL PAIRS
}

\author{
SHU-YEN PAN
}

\begin{abstract}
In this paper, we construct a splitting of the metaplectic cover of the reductive dual pairs of orthogonal and symplectic groups or the reductive dual pairs of unitary groups over a nonarchimedean local field with respect to a generalized lattice model of the Weil representation. We also prove a result concerning the splitting that we construct and the theta dichotomy for unitary group. The splitting plays a very crucial role in the study of theta correspondence for $\boldsymbol{p}$-adic and finite reductive dual pairs.
\end{abstract}

\section{Introduction.}

Let $F$ be a $p$-adic field with odd residual characteristic. Let $D$ be $F$ itself or a quadratic extension of $F$. Let $\mathcal{O}_{D}$ be the ring of integers, $\mathfrak{p}_{D}$ be the maximal ideal in $\mathcal{O}_{D}, \mathbf{f}_{D}$ be the (finite) residue field and $\varpi$ be a prime element in $D$. Let $\mathcal{V}$ (resp. $\mathcal{V}^{\prime}$ ) be a finite-dimensional nondegenerate $\epsilon$-hermitian (resp. $\epsilon^{\prime}$-hermitian) space over $D$ where $\epsilon, \epsilon^{\prime}$ are 1 or -1 and $\epsilon \epsilon^{\prime}=-1$. Let $U(\mathcal{V})$ (resp. $U\left(\mathcal{V}^{\prime}\right)$ ) denote the group of isometries of $\mathcal{V}$ (resp. $\left.\mathcal{V}^{\prime}\right)$. We can define a skew-symmetric $F$-bilinear form on $\mathcal{W}:=\mathcal{V} \otimes_{D} \mathcal{V}^{\prime}$. Then the pair of two groups $\left(U(\mathcal{V}), U\left(\mathcal{V}^{\prime}\right)\right)$ forms a reductive dual pair in the symplectic group $\operatorname{Sp}(\mathcal{W})$. In particular, we have embeddings $\iota_{\mathcal{V}^{\prime}}: U(\mathcal{V}) \rightarrow \operatorname{Sp}(\mathcal{W})$ and $\iota_{\mathcal{V}}: U\left(\mathcal{V}^{\prime}\right) \rightarrow \operatorname{Sp}(\mathcal{W})$.

Let $(M[g], \mathcal{S})$ be a model of the Weil (projective) representation of $\operatorname{Sp}(\mathcal{W})$ with respect to a fixed nontrivial character $\psi$ of $F$. Then there is a twococycle $c: \operatorname{Sp}(\mathcal{W}) \times \operatorname{Sp}(\mathcal{W}) \rightarrow \mathbf{C}^{\times}$associated to $(M[g], \mathcal{S})$ given by

$$
M[g] \circ M\left[g^{\prime}\right]=c\left(g, g^{\prime}\right) M\left[g g^{\prime}\right] .
$$

This two-cocycle $c\left(g, g^{\prime}\right)$ determines an extension

$$
1 \longrightarrow \mathbf{C}^{\times} \longrightarrow \widehat{\operatorname{Sp}(\mathcal{W})} \longrightarrow \mathrm{Sp}(\mathcal{W}) \longrightarrow 1
$$

The group $\widehat{\operatorname{sp}(\mathcal{W})}$ is called the metaplectic cover of $\operatorname{Sp}(\mathcal{W})$. The projective representation $(M[g], \mathcal{S})$ of $\operatorname{Sp}(\mathcal{W})$ can be lifted as an ordinary representation $(\omega(g), \mathcal{S})$ of $\widehat{\operatorname{Sp}(\mathcal{W})}$. 
Let $\widetilde{U(\mathcal{V})}$ denote the inverse image of $\iota \mathcal{V}^{\prime}(U(\mathcal{V}))$ in $\widetilde{\operatorname{Sp}(\mathcal{W})}$ under the above extension. Then $\widetilde{U(\mathcal{V})}$ is also called the metaplectic cover of $U(\mathcal{V})$. A map $\beta_{\mathcal{V}^{\prime}}: U(\mathcal{V}) \rightarrow \mathbf{C}^{\times}$is called a splitting of the metaplectic cover $\widetilde{U(\mathcal{V})}$ if it satisfies

$$
c\left(\iota \mathcal{V}^{\prime}(g), \iota \mathcal{V}^{\prime}\left(g^{\prime}\right)\right)=\beta_{\mathcal{V}^{\prime}}\left(g g^{\prime}\right) \beta_{\mathcal{V}^{\prime}}(g)^{-1} \beta_{\mathcal{V}^{\prime}}\left(g^{\prime}\right)^{-1}
$$

for any $g, g^{\prime} \in U(\mathcal{V})$. Such a splitting determines a group homomorphism $\widetilde{\beta}_{\mathcal{V}^{\prime}}: U(\mathcal{V}) \rightarrow \widetilde{U(\mathcal{V})}$. Suppose that $\beta_{1}, \beta_{2}: U(\mathcal{V}) \rightarrow \mathbf{C}^{\times}$are two splittings of the cocycle $\left.c\right|_{\iota_{\mathcal{V}^{\prime}}(U(\mathcal{V})) \times \iota_{\mathcal{V}^{\prime}}(U(\mathcal{V}))}$. Then it is well-known that the map $\beta_{1} \beta_{2}^{-1}: U(\mathcal{V}) \rightarrow \mathbf{C}^{\times}$is a character of $U(\mathcal{V})$ i.e., two splittings differ by a character of $U(\mathcal{V})$. A reductive dual pair $\left(U(\mathcal{V}), U\left(\mathcal{V}^{\prime}\right)\right)$ is called split if there exist splittings for metaplectic covers $\widehat{U(\mathcal{V})}$ and $\widehat{U\left(\mathcal{V}^{\prime}\right)}$. It is known that the dual pair is always split when $D$ is a quadratic extension of $F$. If $D=F$, one of $\mathcal{V}, \mathcal{V}^{\prime}$ is a quadratic space and the other is a symplectic space. It is also known that the reductive dual pair $\left(U(\mathcal{V}), U\left(\mathcal{V}^{\prime}\right)\right)$ of a symplectic group and an orthogonal group is split if and only if the quadratic space is even-dimensional. Now we suppose that $\left(U(\mathcal{V}), U\left(\mathcal{V}^{\prime}\right)\right)$ is a split reductive dual pair. It is known that $\widetilde{U(\mathcal{V})}$ and $\widehat{U\left(\mathcal{V}^{\prime}\right)}$ commute with each other in $\widetilde{\operatorname{Sp}(\mathcal{W})}$. Therefore $\widetilde{U(\mathcal{V})} \cdot \widetilde{U\left(\mathcal{V}^{\prime}\right)}$ is a subgroup of $\widetilde{\operatorname{Sp}(\mathcal{W})}$. By restricting the representation $(\omega(g), \mathcal{S})$ to $\widetilde{U(\mathcal{V})} \cdot \widetilde{U\left(\mathcal{V}^{\prime}\right)}$ and pulling back to $U(\mathcal{V}) \times U\left(\mathcal{V}^{\prime}\right)$ by the homomorphisms $\widetilde{U(\mathcal{V})} \times \widehat{U\left(\mathcal{V}^{\prime}\right)} \rightarrow \widetilde{U(\mathcal{V})} \cdot \widetilde{U\left(\mathcal{V}^{\prime}\right)}, U(\mathcal{V}) \rightarrow \widetilde{U(\mathcal{V})}$ and $U\left(\mathcal{V}^{\prime}\right) \rightarrow \widehat{U\left(\mathcal{V}^{\prime}\right)}$, there exists a correspondence between irreducible admissible representations of $U(\mathcal{V})$ and $U\left(\mathcal{V}^{\prime}\right)$. This correspondence is called the local theta correspondence. It is proved by R. Howe and J.-L. Waldspurger $[\mathbf{W} \mathbf{p}]$ that this correspondence is one-to-one. (Note that we always assume the characteristic of $\mathbf{f}_{F}$ is odd.)

It is clear that the local theta correspondence for a split reductive dual pair depends on the splittings of the metaplectic covers. It is important to know $\beta_{\mathcal{V}^{\prime}}(g)$ explicitly for a given model $(M[g], \mathcal{S})$ of the Weil representation. If we choose a maximal totally isotropic subspace $Y$ of $\mathcal{W}$, then we get the Schrödinger model $\left(M_{Y}[g], \mathcal{S}(Y)\right)$. A splitting $\beta_{\mathcal{V}^{\prime}}^{Y}(g)$ of the cocycle $c_{Y}\left(g, g^{\prime}\right)$ with respect to the Schrödinger model $\left(M_{Y}[g], \mathcal{S}(Y)\right)$ for a proper chosen $Y$ is obtained by S. Kudla in $[\mathbf{K l}]$ by using the formula of $c_{Y}\left(g, g^{\prime}\right)$ in $[\mathbf{R R}]$. The main task of this work is to investigate the splittings of the metaplectic covers with respect to a generalized lattice model. A generalized lattice model is convenient for the study of the local theta correspondence. For example, it is the model used by Waldspurger to prove that the correspondence is one-to-one. It is also used by $\mathrm{D}$. Manderscheid in $[\mathbf{M d}]$ to study the local theta correspondence for some reductive dual pairs. 
To introduce the generalized lattice model we need a few more definitions. Let $L$ be a good lattice in $\mathcal{V}$ i.e., a lattice such that $\varpi L^{*} \subseteq L \subseteq L^{*}$ where $L^{*}:=\left\{v \in \mathcal{V} \mid \mathbf{h}(v, l) \in \mathfrak{p}_{D}^{\kappa}\right.$ for all $\left.l \in L\right\}$ and $\kappa$ is an integer which will be specified in Subsection 3.1. Then the subgroup $U(\mathcal{V})_{L}$ of elements stabilizing $L$ is a maximal open compact subgroup of $U(\mathcal{V})$. Let $\widetilde{U(\mathcal{V})_{L}}$ denote the inverse image of $\iota \mathcal{V}^{\prime}\left(U(\mathcal{V})_{L}\right)$ in $\widehat{\operatorname{Sp}(\mathcal{W})}$. Let $L^{\prime}$ be a good lattice in $\mathcal{V}^{\prime}$. Define

$$
B:=L^{*} \otimes L^{\prime} \cap L \otimes L^{\prime *} .
$$

Then $B$ is a good lattice in $\mathcal{W}$ with respect to conductoral exponent $\lambda_{F}$ of $\psi$. Then we know that $B^{*} / B$ is a finite symplectic space. We can define the generalized lattice model $\left(M_{B}[g], \mathcal{S}(B)\right)$ of the Weil representation of $\operatorname{Sp}(\mathcal{W})$ where $\mathcal{S}(B)$ is the space of locally constant, compactly supported maps from $\mathcal{W}$ to the space of the Weil representation of the finite symplectic group $\operatorname{Sp}\left(B^{*} / B\right)$ and satisfies some additional conditions. Let $\left(\omega_{B}[g], \mathcal{S}(B)\right)$ be the Weil representation of $\widehat{\operatorname{Sp}(\mathcal{W})}$ lifted from $\left(M_{B}[g], \mathcal{S}(B)\right)$. Let $K_{B}$ denote the stabilizer of $B$ in $\operatorname{Sp}(\mathcal{W})$ and $\widetilde{K}_{B}$ denote the inverse image of $K_{B}$ in $\widetilde{\operatorname{Sp}(\mathcal{W})}$. The advantage of the generalized lattice model is that the restriction of action $M_{B}[g]$ to $K_{B}$ is very simple. In particular, it is known that the cocycle $c_{B}\left(g, g^{\prime}\right)$ with respect to $\left(M_{B}[g], \mathcal{S}(B)\right)$ is trivial when restricted to $K_{B} \times K_{B}$. It is clear that $\iota \mathcal{V}^{\prime}\left(U(\mathcal{V})_{L}\right) \subseteq K_{B}$. Therefore, a map $\zeta: U(\mathcal{V})_{L} \rightarrow \mathbf{C}^{\times}$is a splitting of the metaplectic cover $\widetilde{U(\mathcal{V})_{L}}$ with respect to the generalized lattice model $\left(M_{B}[g], \mathcal{S}(B)\right)$ if and only if $\zeta$ is a (quasi-)character. To take the full advantage of the generalized lattice model, we need to know for which character $\zeta$ the splitting $\zeta: U(\mathcal{V})_{L} \rightarrow \mathbf{C}^{\times}$can be extended to the whole group $U(\mathcal{V})$. We answer this question as follows:

Let $\Psi: \mathcal{S}(B) \rightarrow \mathcal{S}(Y)$ be an isomorphism from a generalized lattice model to a Schrödinger model. Then we can define a function $\alpha_{\mathcal{V}^{\prime}}: U(\mathcal{V}) \rightarrow \mathbf{C}^{\times}$by

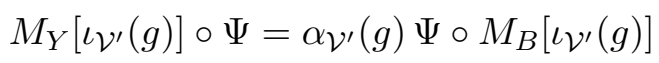

for $g \in U(\mathcal{V})$. Then the function $\alpha_{\mathcal{V}^{\prime}} \beta_{\mathcal{V}^{\prime}}^{Y}$ is a splitting of the cocycle $c_{B}\left(\iota_{\mathcal{V}^{\prime}}(g), \iota_{\mathcal{V}^{\prime}}\left(g^{\prime}\right)\right)$. In general, the whole function $\alpha_{\mathcal{V}^{\prime}} \beta_{\mathcal{V}^{\prime}}^{Y}$ is too complicated to be computed. However, the computation of the restriction $\left.\alpha_{\mathcal{V}^{\prime}} \beta_{\mathcal{V}^{\prime}}^{Y}\right|_{U(\mathcal{V})_{L}}$ is more accessible. In fact, knowing the values of $\left.\alpha_{\mathcal{V}^{\prime}} \beta_{\mathcal{V}^{\prime}}^{Y}\right|_{U(\mathcal{V})_{L}}$ is already adequate to our purpose. We know that $\left.\alpha_{\mathcal{V}^{\prime}} \beta_{\mathcal{V}^{\prime}}^{Y}\right|_{U(\mathcal{V})_{L}}$ is a character of $U(\mathcal{V})_{L}$. Hence $\left.\alpha_{\mathcal{V}^{\prime}} \beta_{\mathcal{V}^{\prime}}^{Y}\right|_{U(\mathcal{V})_{L}}$ factors through the quotient by the commutator subgroup $\left[U(\mathcal{V})_{L}, U(\mathcal{V})_{L}\right]$. From the result in $[\mathbf{P n 1}]$, we know that

$$
U(\mathcal{V})_{L} /\left[U(\mathcal{V})_{L}, U(\mathcal{V})_{L}\right] \simeq U(\mathcal{V})_{L} / S U(\mathcal{V})_{L} \times Q
$$

where $Q$ is a finite group. A more precise statement is in (1.4.b). If we regard $\left.\alpha_{\mathcal{V}} \beta_{\mathcal{V}^{\prime}}^{Y}\right|_{U(\mathcal{V})_{L}}$ as a character of $U(\mathcal{V})_{L} /\left[U(\mathcal{V})_{L}, U(\mathcal{V})_{L}\right]$, we see that the obstruction of $\left.\alpha \mathcal{V}^{\prime} \beta_{\mathcal{V}^{\prime}}^{Y}\right|_{U(\mathcal{V})_{L}}$ to be the restriction of a character of $U(\mathcal{V})$ is on 
the component $Q$. After a long computation, we find that $\left.\alpha_{\mathcal{V}^{\prime}} \beta_{\mathcal{V}^{\prime}}^{Y}\right|_{S U(\mathcal{V})_{L}}=$ $\left.\zeta \mathcal{V}^{\prime}\right|_{S U(\mathcal{V})_{L}}$ where $\zeta_{\mathcal{V}^{\prime}}$ is a character of order two when $U(\mathcal{V})$ is a unitary group and the dimension of $\mathcal{V}^{\prime}$ is odd, and is the trivial character otherwise. A more precise definition of $\zeta_{\mathcal{V}^{\prime}}$ is in Subsection 3.3. Hence $\alpha_{\mathcal{V}^{\prime}} \beta_{\mathcal{V}^{\prime}}^{Y} \zeta_{\mathcal{V}^{\prime}}^{-1}$ is a character of $U(\mathcal{V})_{L}$ and factors through the determinant map. Then there is a character $\xi$ of $U(\mathcal{V})$ such that $\alpha \mathcal{V}^{\prime} \beta_{\mathcal{V}^{\prime}}^{Y} \zeta_{\mathcal{V}^{\prime}}^{-1}=\left.\xi\right|_{U(\mathcal{V})_{L}}$. Define

$$
\beta_{\mathcal{V}^{\prime}}^{L}(g):=\alpha \mathcal{V}^{\prime}(g) \beta_{\mathcal{V}^{\prime}}^{Y}(g) \xi^{-1}(g)
$$

for $g \in U(\mathcal{V})$. Hence we obtain the following our first main result of this paper (cf. Theorem 3.4).

Theorem A. Suppose $L$ is a good lattice in $\mathcal{V}$ and the metaplectic cover $\widetilde{U(\mathcal{V})}$ splits. There exists a splitting $\beta_{\mathcal{V}^{\prime}}^{L}: U(\mathcal{V}) \rightarrow \mathbf{C}^{\times}$of $\widetilde{U(\mathcal{V})}$ such that $\left.\beta_{\mathcal{V}^{\prime}}^{L}\right|_{U(\mathcal{V})_{L}}$ is a character of order two when $U(\mathcal{V})$ is a unitary group and the dimension of $\mathcal{V}^{\prime}$ is odd, and is the trivial character otherwise.

Because the restriction of an irreducible admissible representation to certain maximal open compact subgroups is an important technique to study representations of a $p$-adic reductive group, the splitting of the metaplectic cover that we consider is expected to play an important role in the explicit description of the local theta correspondence. In fact, by using the splitting asserted by Theorem A, we establish in [Pn2] a nice relation of local theta correspondence of depth zero representations of a $p$-adic reductive dual pair and the theta correspondence of some finite reductive dual pairs. The application of this relation to the study of theta dichotomy is the motivation for our second main result (cf. Proposition 10.2).

Theorem B. Let $\left(U(\mathcal{V}), U\left(\mathcal{V}^{ \pm}\right)\right)$be reductive dual pairs of unitary groups where $\mathcal{V}^{ \pm}$are defined by

$$
\epsilon_{E / F}\left((-1)^{\frac{m^{ \pm}\left(m^{ \pm}-1\right)}{2}} \operatorname{det}\left(\mathcal{V}^{\prime \pm}\right)\right)= \pm 1
$$

where $m^{ \pm}$is the dimension of $\mathcal{V}^{ \pm}$. Let $\eta$ be the character of order two of $U(\mathcal{V})$. Let $L\left(\right.$ resp. $\left.L^{ \pm}\right)$be a good lattice in $\mathcal{V}\left(\right.$ resp. $\left.\mathcal{V}^{ \pm}\right)$. Then

$$
\alpha_{\mathcal{V}^{\prime+}}(g) \beta_{\mathcal{V}^{++}}^{Y^{+}}(g)=\eta(g) \alpha_{\mathcal{V}^{\prime-}}(g) \beta_{\mathcal{V}^{\prime-}}^{Y^{-}}(g)
$$

for $g \in U(\mathcal{V})_{L}$.

By applying Theorem B, in [Pn2] we obtain theta dichotomy for finite reductive dual pairs of orthogonal and symplectic groups and pairs of unitary groups from the theta dichotomy of $p$-adic unitary groups in $[\mathbf{H K S}]$. In fact, this application is our motivation to study the splittings of the metaplectic covers with respect to a generalized lattice model.

The contents of this paper are as follows: Section 1 consists of basic definitions and preliminary results that will be used throughout the paper. 
In particular, we state the result from [Pn1] concerning the structure of the commutator subgroups of maximal open compact subgroups of $p$-adic classical groups. In Section 2, we introduce the Schrödinger model and the generalized lattice model of the Weil representation. The material in this section is well-known and can be found in $[\mathbf{R R}]$ and $[\mathbf{W} \mathbf{p}]$. In Section 3, we introduce the splittings of the metaplectic covers and state our main result. The main task of the remaining part of this paper is to prove Proposition 3.3. The proof occupies Sections 6-9 according to unitary groups with respect to an unramified extension, unitary groups with respect to a ramified extension, orthogonal groups and symplectic groups. Sections 4 and 5 consist of lemmas that are needed for the proof in the latter sections. In Section 4, we study the isomorphisms between several models of the Weil representation introduced in Section 2. In Section 5, we have some computations for the reductive dual pairs of unitary groups. In this section, an embedding of an anisotropic unitary group into a unitary group of a split $\epsilon$-hermitian form is modified from $[\mathbf{K l}]$. In Section 10, we prove a proposition which concerns a relation of the splittings and theta dichotomy.

This subject occurs naturally from the study of local theta correspondence via minimal $K$-types in the author's dissertation. The author would like to thank Professor Dan Barbasch for introducing him to this direction. The author also want to thank Professors Jeffrey Adams and Professor Stephen Kudla for their interest in this work. Finally, the author thanks the referee for several useful suggestions to improve the presentation of this paper.

\section{Preliminaries.}

1.1. Notation. Let $F$ be a nonarchimedean local field, $\mathcal{O}_{F}$ be the ring of integers of $F, \mathfrak{p}_{F}$ be the maximal ideal, $\varpi_{F}$ be a prime element, $\mathbf{f}_{F}:=\mathcal{O}_{F} / \mathfrak{p}_{F}$ be the (finite) residue field, $\tau_{F}$ be the identity automorphism of $F$. We assume that the characteristic of $\mathbf{f}_{F}$ is odd throughout this paper. Let $q$ denote the cardinality of $\mathbf{f}_{F}$. Fix a character $\psi$ of $F$ with conductoral exponent $\lambda_{F}$ i.e., $\lambda_{F} \in \mathbf{Z}$ is the smallest integral number such that $\psi_{\mathfrak{p}_{F}^{\lambda_{F}}}$ is trivial. We shall use the notation ord $(\psi)$ to denote the conductoral exponent of $\psi$. Let $\psi_{0}$ be the character of $F$ defined by $\psi_{0}(t):=\psi(t / 2)$ for $t \in F$.

Let $E$ be a quadratic extension of $F, \mathcal{O}_{E}$ be the ring of integers of $E, \varpi_{E}$ be a prime element in $\mathcal{O}_{E}, \mathbf{f}_{E}$ be the residue field of $E, \tau_{E}$ be the nontrivial automorphism of $E$ over $F$. We make the choice such that $\varpi_{E}=\varpi_{F}$ if $E$ is unramified, and $\tau_{E}\left(\varpi_{E}\right)=-\varpi_{E}$ if $E$ is ramified. Let $E^{(1)}$ denote the group of norm one elements in $E^{\times}$i.e., $E^{(1)}:=\left\{t \in E^{\times} \mid t \tau_{E}(t)=1\right\}$. If $E$ is an unramified quadratic extension of $F$, then $\mathbf{f}_{E}$ is a quadratic extension of $\mathbf{f}_{F}$. Similarly, let $\mathbf{f}_{E}^{(1)}$ denote the group of norm one elements in $\mathbf{f}_{E}^{\times}$. We fix $(D, \varpi, \tau)$ to be one of the triples $\left(F, \varpi_{F}, \tau_{F}\right)$ or $\left(E, \varpi_{E}, \tau_{E}\right)$. Let $\mathcal{O}_{D}$ be 
the ring of integers of $D, \mathbf{f}_{D}$ the residue field of $D$. Let $\Pi_{\mathcal{O}_{D}}: \mathcal{O}_{D} \rightarrow \mathbf{f}_{D}$ be the usual quotient map. Let ord be the discrete valuation of $D$ such that $\operatorname{ord}\left(F^{\times}\right)=\mathbf{Z}$.

If $a$ is a real number, the largest integer not greater than $a$ is denoted by $\lfloor a\rfloor$, and the smallest integer not less than $a$ is denoted by $\lceil a\rceil$.

1.2. Classical groups and $\epsilon$-hermitian spaces. Let $\epsilon$ be the number 1 or -1 . Let $\mathcal{V}$ be a (finite-dimensional nondegenerate left) $\epsilon$-hermitian space over $D$ i.e., there exists a map $\mathbf{h}():, \mathcal{V} \times \mathcal{V} \rightarrow D$ such that

$$
\begin{aligned}
\mathbf{h}(x+y, z) & =\mathbf{h}(x, z)+\mathbf{h}(y, z), & \mathbf{h}(x, y+z) & =\mathbf{h}(x, y)+\mathbf{h}(x, z), \\
\mathbf{h}(a x, b y) & =a \mathbf{h}(x, y) \tau(b), & \mathbf{h}(x, y) & =\epsilon \tau(\mathbf{h}(y, x))
\end{aligned}
$$

for any $x, y \in \mathcal{V}$ and $a, b \in D$, and $\mathbf{h}(x, \mathcal{V})=0$ implies $x=0$. A nontrivial vector $v \in \mathcal{V}$ is called isotropic (resp. anisotropic) if $\mathbf{h}(v, v)=0$ (resp. $\mathbf{h}(v, v) \neq 0)$. The space $\mathcal{V}$ is called isotropic if an isotropic vector exists, and is called anisotropic otherwise. The space $\mathcal{V}$ is called a hyperbolic plane if $\mathcal{V}$ is two-dimensional and isotropic. If $\mathcal{V}$ is a hyperbolic plane, a basis $\left\{v_{1}, v_{2}\right\}$ is called a standard basis of $\mathcal{V}$ if it satisfies $\mathbf{h}\left(v_{1}, v_{2}\right)=1$ and $\mathbf{h}\left(v_{1}, v_{1}\right)=$ $\mathbf{h}\left(v_{2}, v_{2}\right)=0$.

Let $U(\mathcal{V}, \mathbf{h})$ or $U(\mathcal{V})$ denote the group of isometries of $(\mathcal{V}, \mathbf{h})$. Let det: $U(\mathcal{V}) \rightarrow D^{\times}$denote the determinant map. Let $S U(\mathcal{V})$ denote the subgroup of $U(\mathcal{V})$ consisting of elements of determinant one. The groups $U(\mathcal{V})$ or $S U(\mathcal{V})$ are called classical groups (over $F$ ). The group $U(\mathcal{V})$ is a symplectic group if $(D, \epsilon)=(F,-1)$, is an orthogonal group if $(D, \epsilon)=(F, 1)$, and is a unitary group if $D=E$.

The definitions above can be applied to a finite field. More precisely, let $\mathbf{f}$ be a finite field of odd characteristic and $\mathbf{d}$ be $\mathbf{f}$ itself or a quadratic extension of $\mathbf{f}$. Let $\tau_{\mathbf{d}}$ denote the usual involution of $\mathbf{d}$ over $\mathbf{f}$. Let $\mathbf{v}$ be a finite-dimensional nondegenerate $\epsilon$-hermitian space over $\mathbf{d}$ for $\epsilon=1$ or -1 . Let $U(\mathbf{v})$ denote the group of isometries of $\mathbf{v}$ and $S U(\mathbf{v})$ denote the subgroup of elements of determinant one. The groups $U(\mathbf{v})$ or $S U(\mathbf{v})$ are called finite classical groups.

1.3. Good lattices in an $\epsilon$-hermitian space. Let $L$ be a lattice in $\mathcal{V}$, that is, a free $\mathcal{O}_{D}$-module whose rank is equal to the dimension of $\mathcal{V}$. Fix an integer $\kappa$. Define

$$
L^{*}:=\left\{v \in \mathcal{V} \mid \mathbf{h}(v, l) \in \mathfrak{p}_{D}^{\kappa} \text { for all } l \in L\right\} .
$$

Therefore $L^{*}$ depends on the integer $\kappa$. Right now we allow $\kappa$ to be arbitrary, but we will impose some condition on $\kappa$ in Subsection 3.1. It is clear that $L^{*}$ is also a lattice in $\mathcal{V}$. The lattice $L$ is called a good lattice if

$$
\varpi L^{*} \subseteq L \subseteq L^{*}
$$


Let $L$ be a good lattice in $\mathcal{V}$. Then $\mathbf{l}^{*}:=L^{*} / L$ and $\mathbf{l}:=L / \varpi L^{*}$ are vector spaces over $\mathbf{f}_{D}$. We can define nondegenerate sesquilinear forms $\mathbf{h}(,)_{\mathbf{l}^{*}}, \mathbf{h}(,)_{\mathbf{l}}$ on $\mathbf{l}^{*}$ and $\mathbf{l}$ respectively by

$$
\begin{aligned}
\left\langle\Pi_{L^{*}}(w), \Pi_{L^{*}}\left(w^{\prime}\right)\right\rangle_{\mathbf{1}^{*}} & :=\Pi_{\mathcal{O}_{D}}\left(\left\langle w, w^{\prime}\right\rangle \varpi^{1-\kappa}\right), \\
\left\langle\Pi_{L}(v), \Pi_{L}\left(v^{\prime}\right)\right\rangle_{\mathbf{l}} & :=\Pi_{\mathcal{O}_{D}}\left(\left\langle v, v^{\prime}\right\rangle \varpi^{-\kappa}\right)
\end{aligned}
$$

where $w, w^{\prime} \in L^{*}, v, v^{\prime} \in L$. Note that the forms $\langle,\rangle_{\mathbf{l}^{*}}$ and $\langle,\rangle_{\mathbf{l}}$ are nondegenerate and depend on the choice of a prime element $\varpi$. The following table is from $[\mathbf{W p}]$, Lemme I.2.

\begin{tabular}{|l|l|l|}
\hline & $\mathbf{h}(,)_{\mathbf{l}^{*}}$ & $\mathbf{h}(,)_{\mathbf{l}}$ \\
\hline$D=F$ & $\epsilon$-symmetric over $\mathbf{f}_{F}$ & $\epsilon$-symmetric over $\mathbf{f}_{F}$ \\
\hline$D=E$, unramified & $\epsilon$-hermitian over $\mathbf{f}_{E}$ & $\epsilon$-hermitian over $\mathbf{f}_{E}$ \\
\hline$D=E$, ramified & $(-1)^{\kappa+1} \epsilon$-symmetric over $\mathbf{f}_{F}$ & $(-1)^{\kappa} \epsilon$-symmetric over $\mathbf{f}_{F}$ \\
\hline
\end{tabular}

Define $\ell(V):=\operatorname{dim}(V)-2 r(V)$ where $V$ is $\mathcal{V}, \mathbf{l}$ or $\mathbf{l}^{*}$ and $r(V)$ denotes the Witt index of $V$. It is straight forward to check that $\ell(\mathcal{V})=\ell(\mathbf{l})=\ell\left(\mathbf{l}^{*}\right)=0$ if $\mathcal{V}$ is a symplectic space; $\ell(\mathbf{l})=\ell\left(\mathbf{l}^{*}\right)=2$ if $\ell(\mathcal{V})=4$ and $\mathcal{V}$ quadratic; one of $\ell(\mathbf{l}), \ell\left(\mathbf{1}^{*}\right)$ is one and the other is two if $\ell(\mathcal{V})=3$ and $\mathcal{V}$ quadratic; either $\ell(\mathbf{l})=\ell\left(\mathbf{l}^{*}\right)=1$, or one of $\ell(\mathbf{l}), \ell\left(\mathbf{l}^{*}\right)$ is zero and the other is two if $\ell(\mathcal{V})=2$ and $\mathcal{V}$ quadratic; one of $\ell(\mathbf{l}), \ell\left(\mathbf{l}^{*}\right)$ is zero and the other is two if $\ell(\mathcal{V})=2$ and $\mathcal{V}$ hermitian with respect to a ramified extension; $\ell(\mathbf{l})=\ell\left(\mathbf{l}^{*}\right)=1$ if $\ell(\mathcal{V})=2$ and $\mathcal{V}$ hermitian with respect to an unramified extension. In particular, we have $\ell(\mathbf{l})+\ell\left(\mathbf{l}^{*}\right)=\ell(\mathcal{V})$ for any cases.

Let $L$ be a good lattice in $\mathcal{V}$. A decomposition $\mathcal{V}=\bigoplus_{i} \mathcal{V}_{i}$ of subspaces is called $L$-admissible if $L=\sum_{i}\left(L \cap \mathcal{V}_{i}\right)$. A basis $\left\{v_{i}\right\}$ of $\mathcal{V}$ is called $L$-admissible if the decomposition $\mathcal{V}=\bigoplus_{i} D v_{i}$ is $L$-admissible. A nondegenerate subspace $\mathcal{V}_{1}$ of $\mathcal{V}$ is called L-admissible if the decomposition $\mathcal{V}=\mathcal{V}_{1} \oplus \mathcal{V}_{1}^{\perp}$ is $L$ admissible where $\mathcal{V}_{1}^{\perp}$ denotes the orthogonal complement of $\mathcal{V}_{1}$ in $\mathcal{V}$. It is clear that we have an $L$-admissible decomposition

$$
\mathcal{V}=\mathcal{V}_{1} \oplus \mathcal{V}_{2}
$$

such that $L \cap \mathcal{V}_{1}=L^{*} \cap \mathcal{V}_{1}$ and $L \cap \mathcal{V}_{2}=\varpi\left(L^{*} \cap \mathcal{V}_{2}\right)$. Then it is clear that $\operatorname{dim}(\mathbf{l})=\operatorname{dim}\left(\mathcal{V}_{1}\right)$ and $\operatorname{dim}\left(\mathbf{l}^{*}\right)=\operatorname{dim}\left(\mathcal{V}_{2}\right)$.

Lemma. (i) An anisotropic space $\mathcal{V}$ has a unique good lattice.

(ii) Suppose that $\mathcal{V}=D v_{1} \oplus D v_{2}$ is a hyperbolic plane with a standard basis $\left\{v_{1}, v_{2}\right\}$ and $\kappa=0$. Let $L_{1}:=\mathcal{O}_{D} v_{1}+\mathcal{O}_{D} v_{2}$ and $L_{2}:=\mathcal{O}_{D} v_{1}+\mathfrak{p}_{D} v_{2}$. Then any good lattice in the hyperplane $\mathcal{V}$ is of the form g. $L_{i}$ for some $g \in U(\mathcal{V})$ and $i=1$ or 2 . 
(iii) For general $\epsilon$-hermitian space $\mathcal{V}$ over $D$ and a good lattice $L$ in $\mathcal{V}$, there is an L-admissible decomposition $\mathcal{V}=\bigoplus_{i} \mathcal{V}_{i}$ as direct sum of nondegenerate subspaces such that each $\mathcal{V}_{i}$ is either one-dimensional or two-dimensional isotropic.

Proof. First suppose that $\mathcal{V}$ is anisotropic. Then we have a decomposition $\mathcal{V}=\bigoplus_{i} D v_{i}$ such that $\mathbf{h}\left(v_{i}, v_{j}\right)=0$ for $i \neq j$. Clearly each subspace $D v_{i}$ has a unique good lattice $L_{i}$ and the lattice $\bigoplus_{i} L_{i}$ is a good lattice in the anisotropic space $\mathcal{V}$. In particular, the decomposition $\mathcal{V}=\bigoplus_{i} D v_{i}$ is $L$-admissible. It is not difficult to derive from Bruhat-Tits result in $[\mathbf{B T}]$ that there is a realization of the building of $U(\mathcal{V})$ such that a vertex of the building corresponds to a good lattice in $\mathcal{V}$. Hence the assertion that $\bigoplus_{i} L_{i}$ is the only good lattice in $\mathcal{V}$ follows from the fact that the building of $U(\mathcal{V})$ is a single point.

A proof of (ii) and (iii) can be found in [Pn3] Lemma 4.2.

From the description in this subsection and the previous lemma we see that a good lattice in a $2 n$-dimensional symplectic space $\mathcal{W}$ with $\kappa=0$ is equivalent to one of the following

$$
\begin{aligned}
L_{i}:=\mathfrak{p}_{F} x_{1}+\mathfrak{p}_{F} x_{2}+\cdots+\mathfrak{p}_{F} x_{i} & +\mathcal{O}_{F} x_{i+1}+\cdots \\
& +\mathcal{O}_{F} x_{n}+\mathcal{O}_{F} y_{n}+\mathcal{O}_{F} y_{n-1}+\cdots+\mathcal{O}_{F} y_{1}
\end{aligned}
$$

where $i=0, \ldots, n$ and $\left\{x_{1}, \ldots, x_{n}, y_{1} \ldots, y_{n}\right\}$ is a self-dual basis of $\mathcal{W}$. Note that $L_{0}^{*}=L_{0}$ and $L_{n}^{*}=\varpi_{F}^{-1} L_{n}$.

\subsection{Commutator subgroups of maximal open compact subgroups.} Let $L$ be a good lattice in $\mathcal{V}$. Define

$$
U(\mathcal{V})_{L}:=\{g \in U(\mathcal{V}) \mid g . L=L\} .
$$

It is known that $U(\mathcal{V})_{L}$ is a maximal open compact subgroup of $U(\mathcal{V})$. Moreover it is also known that every maximal open compact subgroup of $U(\mathcal{V})$ is equal to $U(\mathcal{V})_{L}$ for some good lattice $L$ in $\mathcal{V}($ cf. $[\mathbf{H} \mathbf{j}])$. Define $S U(\mathcal{V})_{L}:=U(\mathcal{V})_{L} \cap S U(\mathcal{V})$.

If $G$ is a group, let $[G, G]$ denote the commutator subgroup of $G$. From [Pn1] Theorem 3.1, we have the following isomorphism

$$
\begin{aligned}
U(\mathcal{V})_{L} /\left[U(\mathcal{V})_{L}, U(\mathcal{V})_{L}\right] \simeq & \left(U(\mathcal{V})_{L} / S U(\mathcal{V})_{L}\right) \\
& \times\left(S\left(U(\mathbf{l}) \times U\left(\mathbf{l}^{*}\right)\right) /\left(S U(\mathbf{l}) \times S U\left(\mathbf{l}^{*}\right)\right)\right) \\
& \times(S U(\mathbf{l}) /[U(\mathbf{l}), U(\mathbf{l})]) \times\left(S U\left(\mathbf{l}^{*}\right) /\left[U\left(\mathbf{l}^{*}\right), U\left(\mathbf{l}^{*}\right)\right]\right)
\end{aligned}
$$

where $S\left(U(\mathbf{l}) \times U\left(\mathbf{l}^{*}\right)\right)$ denotes the subgroup of $U(\mathbf{l}) \times U\left(\mathbf{l}^{*}\right)$ consisting of elements $\left(g_{1}, g_{2}\right)$ such that $\operatorname{det}\left(g_{1}\right) \operatorname{det}\left(g_{2}\right)=1$. It is obvious that $U(\mathcal{V})_{L} / S U(\mathcal{V})_{L}$ 
is trivial if $U(\mathcal{V})$ is a symplectic group, is $\mathbf{Z} / 2 \mathbf{Z}$ if $U(\mathcal{V})$ is an orthogonal group, is $E^{(1)}$ if $U(\mathcal{V})$ is a unitary group. It is also obvious that $S\left(U(\mathbf{l}) \times U\left(\mathbf{l}^{*}\right)\right) /\left(S U(\mathbf{l}) \times S U\left(\mathbf{l}^{*}\right)\right)$ is trivial if one of $\mathbf{l}, \mathbf{l}^{*}$ is trivial or a symplectic space, is $\mathbf{Z} / 2 \mathbf{Z}$ if both $U(\mathbf{l}), U\left(\mathbf{l}^{*}\right)$ are orthogonal groups, and is $\mathbf{f}_{E}^{(1)}$ if both $U(\mathbf{l}), U\left(\mathbf{l}^{*}\right)$ are unitary groups.

The structure of commutator subgroups of finite classical groups is wellknown and can be described as follows. Let $U(\mathbf{v})$ be a classical group over a finite field $\mathbf{f}$ with odd characteristic. Then

$$
\begin{aligned}
& S U(\mathbf{v}) /[U(\mathbf{v}), U(\mathbf{v})] \\
& \simeq \begin{cases}1, & \text { if }\left(\tau_{\mathbf{d}}, \epsilon\right)=(\mathrm{id},-1), q>3 \text { or } \operatorname{dim}(\mathbf{v}) \geq 4 ; \\
\mathbf{Z} / 3 \mathbf{Z}, & \text { if }\left(\tau_{\mathbf{d}}, \epsilon\right)=(\mathrm{id},-1), q=3 \text { and } \operatorname{dim}(\mathbf{v})=2 ; \\
1, & \text { if }\left(\tau_{\mathbf{d}}, \epsilon\right)=(\mathrm{id}, 1) \text { and } \operatorname{dim}(\mathbf{v})=1 \\
\mathbf{Z} / 2 \mathbf{Z}, & \text { if }\left(\tau_{\mathbf{d}}, \epsilon\right)=(\mathrm{id}, 1) \text { and } \operatorname{dim}(\mathbf{v}) \geq 2 ; \\
1, & \text { if } \tau_{\mathbf{d}} \neq \mathrm{id} .\end{cases}
\end{aligned}
$$

This result can be found, for example, in [HO] Theorem 6.4.26 and $\S 6.4 \mathrm{G}$.

By (1.4.b) and (1.4.c), the quotient $S U(\mathcal{V})_{L} /\left[U(\mathcal{V})_{L}, U(\mathcal{V})_{L}\right]$ is well understood. The set of representatives of $\left[U(\mathcal{V})_{L}, U(\mathcal{V})_{L}\right]$-cosets in $S U(\mathcal{V})_{L}$ can be described as follows. These descriptions are important for the results in Sections 6-9.

(i) Suppose that $\mathcal{V}$ is a symplectic space. We know that $S U(\mathcal{V})_{L} /\left[U(\mathcal{V})_{L}\right.$, $\left.U(\mathcal{V})_{L}\right]$ is nontrivial only if $q=3$. So we assume that $q=3$. From (1.4.c), $S U(\mathbf{l}) /[U(\mathbf{l}), U(\mathbf{l})]$ is not trivial if and only if $\mathcal{V}_{1}$ is two-dimensional. In this case, let $\left\{v_{1}, v_{2}\right\}$ be a standard basis of $\mathcal{V}_{1}$. If $L=$ $\mathcal{O}_{F} v_{1}+\mathcal{O}_{F} v_{2}$, the image of the element $\left[\begin{array}{ll}1 & 1 \\ 0 & 1\end{array}\right]$ in $S U(\mathcal{V})_{L} /\left[U(\mathcal{V})_{L}\right.$, $\left.U(\mathcal{V})_{L}\right]$ generates $S U(\mathbf{l}) /[U(\mathbf{l}), U(\mathbf{l})]$. We have a similar description when $\mathbf{l}^{*}$ is two-dimensional.

(ii) Suppose that $\mathcal{V}$ is a quadratic space. Let $\mathcal{V}=\mathcal{V}_{1} \oplus \mathcal{V}_{2}$ be the decomposition in (1.3.c).

(ii.1) We know that $S\left(U(\mathbf{l}) \times U\left(\mathbf{l}^{*}\right)\right) /\left(S U(\mathbf{l}) \times S U\left(\mathbf{l}^{*}\right)\right)$ is nontrivial if and only if both $\mathcal{V}_{1}, \mathcal{V}_{2}$ are nontrivial. Assume that we are in this case. For $i=1$ or 2 , if $\mathcal{V}_{i}$ is isotropic, choose an $L$-admissible hyperbolic plane $\mathcal{V}_{i}^{0}$ in $\mathcal{V}_{i}$ and let $g_{i}:=\left[\begin{array}{cc}0 & k \\ k^{-1} & 0\end{array}\right] \in U\left(\mathcal{V}_{i}^{0}\right) \subseteq U(\mathcal{V})$ for some $k \in F^{\times}$such that $g_{i} \in U(\mathcal{V})_{L}$. If $\mathcal{V}_{i}$ is anisotropic, choose an $L$-admissible one-dimensional nondegenerate subspace $\mathcal{V}_{i}^{0}$ of $\mathcal{V}_{i}$ and let $g_{i}:=-1 \in U\left(\mathcal{V}_{i}^{0}\right) \subseteq U(\mathcal{V})$. Therefore $g_{i}$ belongs $U(\mathcal{V})_{L}$ and $g:=g_{1} g_{2}$ belongs to $S U(\mathcal{V})_{L}$. Moreover the image of 
$g$ in $S U(\mathcal{V})_{L} /\left[U(\mathcal{V})_{L}, U(\mathcal{V})_{L}\right]$ generates $S\left(U(\mathbf{l}) \times U\left(\mathbf{l}^{*}\right)\right) /(S U(\mathbf{l}) \times$ $\left.S U\left(\mathbf{l}^{*}\right)\right)$.

(ii.2) We know that $S U(\mathbf{l}) /[U(\mathbf{l}), U(\mathbf{l})]$ is nontrivial if and only if the dimension of $\mathcal{V}_{1}$ is greater than 1 . Assume that we are in this case. If $\mathcal{V}_{1}$ is isotropic, choose an $L$-admissible hyperbolic plane $\mathcal{V}_{i}^{0}$ in $\mathcal{V}_{i}$ and let $g:=\left[\begin{array}{cc}k & 0 \\ 0 & k^{-1}\end{array}\right] \in U\left(\mathcal{V}_{i}^{0}\right) \subseteq U(\mathcal{V})$ for $k \in F^{\times}$such that $\operatorname{ord}(k)=0$ and $k$ is not a square. If $\mathcal{V}_{1}$ is anisotropic, then $\mathcal{V}_{1}$ must be two-dimensional and $\mathcal{V}_{1}$ has two $L$-admissible subspaces $F v_{1}, F v_{2}$ such that $\mathbf{h}\left(v_{1}, v_{1}\right) \mathbf{h}\left(v_{2}, v_{2}\right)$ is not a square. Let $g_{i}$ be the element such that $\left.g_{i}\right|_{F v_{i}}=-1$ and $\left.g_{i}\right|_{\left(F v_{i}\right)^{\perp}}=1$. Therefore we know that $g:=g_{1} g_{2}$ belongs to $S U(\mathcal{V})_{L}$ and the image of $g$ in $S U(\mathcal{V})_{L} /\left[U(\mathcal{V})_{L}, U(\mathcal{V})_{L}\right]$ generates $S U(\mathbf{l}) /[U(\mathbf{l}), U(\mathbf{l})]$. We have a similar description for $S U\left(\mathbf{l}^{*}\right) /\left[U\left(\mathbf{l}^{*}\right) U\left(\mathbf{l}^{*}\right)\right]$.

(iii) Suppose that $\mathcal{V}$ is an $\epsilon$-hermitian space over an unramified quadratic extension $E$ of $F$. The quotient $S U(\mathcal{V})_{L} /\left[U(\mathcal{V})_{L}, U(\mathcal{V})_{L}\right]$ is nontrivial if and only if both $\mathcal{V}_{1}, \mathcal{V}_{2}$ are nontrivial. Assume that we are in this case. If $\mathcal{V}_{i}$ is isotropic, choose an $L$-admissible hyperbolic plane $\mathcal{V}_{i}^{0} \subseteq \mathcal{V}_{i}$ and $g_{i}(k):=\left[\begin{array}{cc}\tau(k) & 0 \\ 0 & k^{-1}\end{array}\right] \in U\left(\mathcal{V}_{i}^{0}\right) \subseteq U(\mathcal{V})$ for some $k \in \mathcal{O}_{E}^{\times}-\mathfrak{p}_{E}^{\times}$. If $\mathcal{V}_{i}$ is anisotropic, choose an $L$-admissible one-dimensional nondegenerate subspace $\mathcal{V}_{i}^{0}$ of $\mathcal{V}_{i}$ and let $g_{i}(k):=\tau(k) k^{-1} \in U\left(\mathcal{V}_{i}^{0}\right) \subseteq U(\mathcal{V})$ for $k$ given as above. Then the element $g:=g_{1}(k) g_{2}\left(k^{-1}\right)$ belongs to $S U(\mathcal{V})_{L}$ and its image in $S U(\mathcal{V})_{L} /\left[U(\mathcal{V})_{L}, U(\mathcal{V})_{L}\right]$ generates $S(U(\mathbf{l}) \times$ $\left.U\left(\mathbf{l}^{*}\right)\right) /\left(S U(\mathbf{l}) \times S U\left(\mathbf{l}^{*}\right)\right)$ for a proper chosen $k$.

(iv) Suppose that $\mathcal{V}$ is an $\epsilon$-hermitian space over a ramified quadratic extension $E$ of $F$. Write $E=F(\sqrt{\Delta})$ for some non-square element $\Delta \in F^{\times}$. Without loss of generality, we assume that $\mathbf{l}$ is a quadratic space and $\mathbf{l}^{*}$ is a symplectic space. Assume that the dimension of $\mathbf{l}$ is greater than 1. If $\mathcal{V}_{1}$ is isotropic, choose an $L$-admissible hyperbolic plane $\mathcal{V}_{1}^{0}$ in $\mathcal{V}_{1}$ and let $g:=\left[\begin{array}{cc}\tau(k) & 0 \\ 0 & k^{-1}\end{array}\right] \in U\left(\mathcal{V}_{1}^{0}\right) \subseteq U(\mathcal{V})$ for $k \in F^{\times}$such that $\operatorname{ord}(k)=0$ and $k$ is not a square. If $\mathcal{V}_{1}$ is anisotropic, then $\mathcal{V}_{1}$ must be two-dimensional and $\mathcal{V}_{1}$ has two $L$-admissible subspaces $E v_{1}, E v_{2}$ such that $\left(\Delta, \mathbf{h}\left(v_{1}, v_{1}\right) \mathbf{h}\left(v_{2}, v_{2}\right)\right)_{F}=-1$ where $(,)_{F}$ denotes the Hilbert symbol. Let $g_{i}$ be the element such that $\left.g_{i}\right|_{E v_{i}}=-1$ and $\left.g_{i}\right|_{\left(E v_{i}\right)^{\perp}}=1$. Therefore, we know that $g:=g_{1} g_{2}$ belongs to $S U(\mathcal{V})_{L}$ and the image of $g$ in $S U(\mathcal{V})_{L} /\left[U(\mathcal{V})_{L}, U(\mathcal{V})_{L}\right]$ generates $S U(\mathbf{l}) /[U(\mathbf{l}), U(\mathbf{l})]$. If $q=3$ and $\operatorname{dim}\left(\mathbf{l}^{*}\right)=2$, then $S U\left(\mathbf{l}^{*}\right) /\left[U\left(\mathbf{l}^{*}\right), U\left(\mathbf{l}^{*}\right)\right]$ is nontrivial. We have an analogous description of the element $g$ as in (i) such that the image of $g$ in $S U(\mathcal{V})_{L} /\left[U(\mathcal{V})_{L}, U(\mathcal{V})_{L}\right]$ generates $S U\left(\mathbf{l}^{*}\right) /\left[U\left(\mathbf{l}^{*}\right), U\left(\mathbf{l}^{*}\right)\right]$. 
1.5. Weil index of a character of second degree. In this subsection, we state some well-known facts on the Weil index of a character of second degree which will be used in latter sections. For $a \in \mathbf{f}_{F}^{\times}$, define

$$
\left(\frac{a}{\mathbf{f}_{F}}\right)=\left\{\begin{aligned}
1, & \text { if } a \text { is a square } \\
-1, & \text { otherwise. }
\end{aligned}\right.
$$

For $a \in F^{\times}$, write $a=\varpi_{F}^{\text {ord }(a)} u$ such that $u$ is a unit of $\mathcal{O}_{F}$. Let $\bar{a}$ denote the element $\Pi_{\mathcal{O}_{F}}(u) \in \mathbf{f}_{F}^{\times}$. Suppose that $\psi$ is a character of $F$. Let $\bar{\psi}$ be the nontrivial character of $\mathbf{f}_{F}$ defined by $\bar{\psi}\left(\Pi_{\mathcal{O}_{F}}(t)\right):=\psi\left(t \varpi_{F}^{\operatorname{ord}(\psi)-1}\right)$. We should notice that $\bar{a}$ and $\bar{\psi}$ depends on the choice of the prime element $\varpi_{F}$. Define $\overline{\operatorname{ord}}(\psi)$ the parity of $\operatorname{ord}(\psi)$ i.e.,

$$
\overline{\operatorname{ord}}(\psi)= \begin{cases}0, & \text { if } \operatorname{ord}(\psi) \text { is even; } \\ 1, & \text { if } \operatorname{ord}(\psi) \text { is odd }\end{cases}
$$

Define $\overline{\operatorname{ord}}(a)$ similarly. For $a \in F^{\times}$, let $\gamma_{F}(\psi), \gamma_{F}(a, \psi), \gamma_{\mathbf{f}_{F}}(\bar{\psi}), \gamma_{\mathbf{f}_{F}}(\bar{a}, \bar{\psi})$ be as defined in $[\mathbf{R R}]$ appendix. The following properties are well-known:

(1) $\gamma_{\mathbf{f}_{F}}(\bar{\psi})^{2}=\left(\frac{-1}{\mathbf{f}_{F}}\right)$,

(2) $\gamma_{\mathbf{f}_{F}}(\bar{a}, \bar{\psi})=\left(\frac{\bar{a}}{\mathbf{f}_{F}}\right)$ for $a \in F^{\times}$,

(3) $\gamma_{F}(\psi)=\gamma_{\mathbf{f}_{F}}(\bar{\psi})^{\overline{\operatorname{ord}}(\psi)}$,

(4) $\gamma_{F}(a, \psi) \gamma_{F}(b, \psi)=\gamma_{F}(a b, \psi)(a, b)_{F}$ for $a, b \in F^{\times}$,

(5) $\gamma_{F}\left(a b^{2}, \psi\right)=\gamma_{F}(a, \psi)$ for $a, b \in F^{\times}$,

(6) $\gamma_{F}(a, \psi)=\left(\left(\frac{\bar{a}}{\mathbf{f}_{F}}\right) \gamma_{\mathbf{f}_{F}}(\bar{\psi})\right)^{\overline{\operatorname{ord}}(a)}\left(\frac{\bar{a}}{\mathbf{f}_{F}}\right)^{\overline{\operatorname{ord}}(\psi)}\left(\frac{-1}{\mathbf{f}_{F}}\right)^{\overline{\operatorname{ord}}(a) \overline{\operatorname{ord}}(\psi)}$ for $a \in F^{\times}$.

The reference is the appendix of $[\mathbf{R R}]$. Note that the formula for $\gamma_{F}(a, \psi)$ in $[\mathbf{R R}]$ Proposition A.11 is not correct. We note here that the right hand sides of (3) and (6) do not depend on the choice of the prime element $\varpi_{F}$ although the terms $\bar{a}$ and $\bar{\psi}$ do.

\section{Several models of the Weil representation.}

In this section, we introduce two special realizations of the Weil representation, namely the Schrödinger model and the generalized lattice model. These realizations are well-known. They can be found in $[\mathbf{M V W}],[\mathbf{R R}]$ or [Wp].

2.1. Weil representations and the metaplectic covers. Let $(\mathcal{W},\langle\langle\rangle)$, be a symplectic space over $F$. Define the Heisenberg group $\mathrm{H}(\mathcal{W})$ (associated to $(\mathcal{W},\langle\langle\rangle)$,$) to be the group with underlying set \mathcal{W} \times F$ and with multiplication given by

$$
\left(w_{1}, t_{1}\right) \cdot\left(w_{2}, t_{2}\right):=\left(w_{1}+w_{2}, t_{1}+t_{2}+\frac{1}{2}\left\langle\left\langle w_{1}, w_{2}\right\rangle\right\rangle\right),
$$

where $w_{1}, w_{2} \in \mathcal{W}$ and $t_{1}, t_{2} \in F$. The center of $\mathrm{H}(\mathcal{W})$ is $\{0\} \times F$, which will be identified with $F$. 
Fix a nontrivial character $\psi$ of $F$. By the Stone-Von Neumann theorem, there is a unique (up to equivalence) irreducible representation $\left(\rho_{\psi}, \mathcal{S}\right)$ of $\mathrm{H}(\mathcal{W})$ with central character $\psi$. The symplectic group $\operatorname{Sp}(\mathcal{W})$ acts on $\mathrm{H}(\mathcal{W})$ by $g .(w, t):=(g . w, t)$. Define the metaplectic cover $\widehat{\operatorname{Sp}(\mathcal{W})}$ of $\operatorname{Sp}(\mathcal{W})$ to be the topological subgroup of $\operatorname{Sp}(\mathcal{W}) \times \operatorname{Aut}(\mathcal{S})$ consisting of the pairs $(g, M[g])$ where $M[g]$ satisfies

$$
M[g] \circ \rho_{\psi}(h)=\rho_{\psi}(g . h) \circ M[g]
$$

for $g \in \operatorname{Sp}(\mathcal{W}), M[g] \in \operatorname{Aut}(\mathcal{S})$ and $h \in \mathrm{H}(\mathcal{W})$. It is clear that $(g, M[g]) \in$ $\widehat{\operatorname{Sp}(\mathcal{W})}$ implies $(g, z M[g]) \in \widehat{\operatorname{Sp}(\mathcal{W})}$ for any $z \in \mathbf{C}^{\times}$. There is a short exact sequence of group homomorphisms

$$
1 \longrightarrow \mathbf{C}^{\times} \stackrel{\alpha}{\longrightarrow} \widetilde{\operatorname{Sp}(\mathcal{W})} \stackrel{\beta}{\longrightarrow} \operatorname{Sp}(\mathcal{W}) \longrightarrow 1
$$

where $\alpha: z \mapsto(1, z 1)$ and $\beta:(g, M[g]) \mapsto g$. The metaplectic group $\widetilde{\operatorname{Sp}(\mathcal{W})}$ comes equipped with a representation $\omega_{\psi}$ on $\mathcal{S}$ given by

$$
\omega_{\psi}(g, M[g]):=M[g] .
$$

The representation $\left(\omega_{\psi}(g), \mathcal{S}\right)$ of $\widetilde{\operatorname{Sp}(\mathcal{W})}$ or the projective representation $(M[g], \mathcal{S})$ of $\operatorname{Sp}(\mathcal{W})$ is called the Weil representation or the oscillator representation.

Let $\left(\rho_{\psi}, \mathcal{S}\right)$ be an irreducible admissible representation of the Heisenberg group $\mathrm{H}(\mathcal{W})$ with the central character $\psi$. Suppose that a special choice of $M[g]$ satisfying (2.1.b) is given for each $g \in \mathrm{Sp}(\mathcal{W})$. By the Stone-Von Neumann theorem, the map $M[g] \circ M\left[g^{\prime}\right]$ is a constant multiple of $M\left[g g^{\prime}\right]$ for $g, g^{\prime} \in \operatorname{Sp}(\mathcal{W})$. Then the function $c: \operatorname{Sp}(\mathcal{W}) \times \operatorname{Sp}(\mathcal{W}) \rightarrow \mathbf{C}^{\times}$defined by

$$
M[g] \circ M\left[g^{\prime}\right]=c\left(g, g^{\prime}\right) M\left[g g^{\prime}\right]
$$

is a two-cocycle of $\operatorname{Sp}(\mathcal{W})$.

2.2. Schrödinger model and Ranga-Rao cocycle. Let $\mathcal{W}=X+Y$ be a complete polarization i.e., both $X, Y$ are totally isotropic and are orthogonal complements of each other. Let $\mathcal{S}(Y)$ be the space of complex valued, locally constant, compactly supported functions on $Y$. For $(x+y, t) \in \mathrm{H}(\mathcal{W})$, define a homomorphism $\rho_{\psi}^{Y}: \mathrm{H}(\mathcal{W}) \rightarrow \mathcal{S}(Y)$ by

$$
\left(\rho_{\psi}^{Y}(x+y, t) . f\right)\left(y^{\prime}\right)=\psi\left(\left\langle\frac{y}{2}+y^{\prime}, x\right\rangle+t\right) f\left(y+y^{\prime}\right)
$$

for any $f \in \mathcal{S}(Y), x \in X, t \in F$, and $y, y^{\prime} \in Y$. For $g \in \operatorname{Sp}(\mathcal{W})$, write $g=\left[\begin{array}{ll}a & b \\ c & d\end{array}\right]$ according to the complete polarization $\mathcal{W}=X+Y$, where $a \in \operatorname{Hom}(X, X), b \in \operatorname{Hom}(Y, X), c \in \operatorname{Hom}(X, Y), d \in \operatorname{Hom}(Y, Y)$. Let $a^{*}$, $b^{*}, c^{*}, d^{*}$ denote the dual maps of $a, b, c, d$ respectively i.e., $a^{*} \in \operatorname{Hom}(Y, Y)$, 
$b^{*} \in \operatorname{Hom}(Y, X), c^{*} \in \operatorname{Hom}(X, Y), d^{*} \in \operatorname{Hom}(X, X)$ are the unique maps such that

$$
\begin{aligned}
&\langle a . x, y\rangle\rangle=\left\langle\left\langle x, a^{*} \cdot y\right\rangle, \quad\langle\langle x, d . y\rangle\rangle\right.=\left\langle\left\langle d^{*} \cdot x, y\right\rangle\right\rangle, \\
&\left\langle\left\langle b . y, y^{\prime}\right\rangle\right\rangle=\left\langle\left\langle y,-b^{*} \cdot y^{\prime}\right\rangle\right\rangle, \quad\left\langle\left\langle x, c . x^{\prime}\right\rangle\right\rangle=\left\langle\left\langle-c^{*} \cdot x, x^{\prime}\right\rangle\right\rangle
\end{aligned}
$$

for any $x, x^{\prime} \in X$ and any $y, y^{\prime} \in Y$. Then we can check $d^{*} a-b^{*} c=1$, $a^{*} d-c^{*} b=1, d^{*} b=b^{*} d, a^{*} b=b^{*} a, a^{*} c=c^{*} a, d^{*} c=c^{*} d$, and $g^{-1}=$ $\left[\begin{array}{cc}d^{*} & -b^{*} \\ -c^{*} & a^{*}\end{array}\right]$. For each $g \in \operatorname{Sp}(\mathcal{W})$, a measure $\mu_{g}$ on the space $X / \operatorname{Ker}(c)$ is given in $[\mathbf{R R}]$ where $\operatorname{Ker}(c)$ denotes the kernel of the homomorphism $c: X \rightarrow Y$. Then define $M_{Y}[g] \in \operatorname{Aut}(\mathcal{S}(Y))($ cf. $[\mathbf{R R}]$ Lemma 3.2) by

$$
\begin{aligned}
& \left(M_{Y}[g] . f\right)(y) \\
& =\int_{X / \operatorname{Ker}(c)} \psi\left(\frac { 1 } { 2 } \left\langle\left\langle a^{*} \cdot y,-b^{*} \cdot y\right\rangle+\left\langle\left\langle c^{*} \cdot x, b^{*} \cdot y\right\rangle\right\rangle\right.\right. \\
& \quad+\frac{1}{2}\left\langle\left\langle-c^{*} \cdot x, d^{*} \cdot x\right\rangle\right) f\left(-c^{*} \cdot x+a^{*} \cdot y\right) d \mu_{g}(x)
\end{aligned}
$$

for $f \in \mathcal{S}(Y)$. One can check that $\rho_{\psi}^{Y}(h)$ and $M_{Y}[g]$ satisfy (2.1.b) for any $g \in \operatorname{Sp}(\mathcal{W})$ and $h \in \mathrm{H}(\mathcal{W})$ (cf. [MVW] p. 40). The realization $\left(M_{Y}[g], \mathcal{S}(Y)\right)$ is called a Schrödinger model of the Weil representation of $\operatorname{Sp}(\mathcal{W})$. This cocycle $c_{Y}\left(g, g^{\prime}\right)$ defined with respect to $M_{Y}[g]$ is called the Ranga-Rao cocycle and it is computed explicitly in [RR] Theorem 4.1.

2.3. Generalized lattice model. The Schrödinger model is also defined for a finite symplectic group. We know that the metaplectic cover of a finite symplectic group splits. Therefore the Weil representation of a finite symplectic group is an ordinary representation.

Let $B$ be a good lattice in $\mathcal{W}$ (with respect to the integer $\lambda_{F}$ ) and $\mathbf{b}^{*}$ be the quotient $B^{*} / B$ and $\Pi_{B^{*}}$ be the quotient map $B^{*} \rightarrow \mathbf{b}^{*}$. We know that $\mathbf{b}^{*}$ is a vector space over $\mathbf{f}_{F}$ with a skew-symmetric form $\left\langle\langle,\rangle_{\mathbf{b}^{*}}\right.$ on $\mathbf{b}^{*}$ by

$$
\left\langle\left\langle\Pi_{B^{*}}(w), \Pi_{B^{*}}\left(w^{\prime}\right)\right\rangle_{\mathbf{b}^{*}}:=\Pi_{\mathcal{O}_{F}}\left(\left\langle w, w^{\prime}\right\rangle\right\rangle \varpi_{F}^{1-\lambda_{F}}\right)
$$

for $w, w^{\prime} \in B^{*}$. Let $\mathrm{H}\left(\mathbf{b}^{*}\right):=\mathbf{b}^{*} \times \mathbf{f}_{F}$ be the Heisenberg group associated to the finite symplectic space $\left(\mathbf{b}^{*},\langle\langle,\rangle\rangle_{\mathbf{b}^{*}}\right)$. Let $\bar{\psi}$ denote the character of $\mathbf{f}_{F}$ as defined in Subsection 1.5. Let $\left(\bar{\omega}_{\bar{\psi}}, S\right)$ be a Schrödinger model of the Weil representation of the finite symplectic groups $\operatorname{Sp}\left(\mathbf{b}^{*}\right)$ associated to the data $\left(\mathbf{b}^{*},\left\langle\langle,\rangle_{\mathbf{b}^{*}}, \bar{\psi}\right)\right.$. Let $\bar{\rho}_{\bar{\psi}}$ denote the representation of $\mathrm{H}\left(\mathbf{b}^{*}\right)$ with the central character $\bar{\psi}$ on the space $S$ by the Stone-Von Neumann theorem. Define $\mathrm{H}\left(B^{*}\right):=B^{*} \times \mathfrak{p}_{F}^{\lambda_{F}-1}$. It is easy to check that $\mathrm{H}\left(B^{*}\right)$ is a subgroup of $\mathrm{H}(\mathcal{W})$. There is a homomorphism

$$
\Pi_{\mathrm{H}\left(B^{*}\right)}: \mathrm{H}\left(B^{*}\right) \rightarrow \mathrm{H}\left(\mathbf{b}^{*}\right) \quad \text { by } \quad(b, t) \mapsto\left(\Pi_{B^{*}}(b), \Pi_{\mathcal{O}_{F}}\left(t \varpi_{F}^{1-\lambda_{F}}\right)\right) .
$$


Let $K_{B}$ be the stabilizer of $B$ in $\operatorname{Sp}(\mathcal{W})$, and

$$
K_{B}^{\prime}:=\left\{g \in K_{B} \mid(g-1) \cdot B^{*} \subseteq B\right\} .
$$

It is clear that $K_{B}^{\prime}$ is a normal subgroup of $K_{B}$ and $K_{B} / K_{B}^{\prime}$ is isomorphic to $\operatorname{Sp}\left(\mathbf{b}^{*}\right)$. Let $\widetilde{\rho}_{\psi}$ denote the representation of $\mathrm{H}\left(B^{*}\right)$ inflated from $\bar{\rho}_{\bar{\psi}}$ by the projection $\Pi_{\mathrm{H}\left(B^{*}\right)}$, and $\widetilde{\omega}_{\psi}$ denote the representation of $K_{B}$ inflated from $\bar{\omega}_{\bar{\psi}}$ by the projection $K_{B} \rightarrow K_{B} / K_{B}^{\prime}$. Let $\mathcal{S}(B)$ denote the space of locally constant, compactly supported maps $f: \mathcal{W} \rightarrow S$ such that

$$
f(b+w)=\psi\left(\frac{1}{2}\langle\langle w, b\rangle\rangle\right) \widetilde{\rho}_{\psi}(b) \cdot(f(w))
$$

for $w \in \mathcal{W}$, and $b \in B^{*}$. For $h:=(w, t) \in \mathrm{H}(\mathcal{W})$, define $\rho_{\psi}^{B}(h) \in \operatorname{Aut}(\mathcal{S}(B))$ by

$$
\left(\rho_{\psi}^{B}(w, t) \cdot f\right)\left(w^{\prime}\right):=\psi\left(\frac{1}{2}\left\langle\left\langle w^{\prime}, w\right\rangle\right\rangle+t\right) f\left(w+w^{\prime}\right)
$$

for $f \in \mathcal{S}(B)$. For $g \in \operatorname{Sp}(\mathcal{W})$, we define $M_{B}[g] \in \operatorname{Aut}(\mathcal{S}(B))$ by

$$
\left(M_{B}[g] . f\right)(w):=\int_{B^{*}} \psi\left(\frac{1}{2}\langle\langle b, w\rangle) \widetilde{\rho}_{\psi}\left(b^{-1}\right) \cdot f\left(g^{-1} \cdot(b+w)\right) d b\right.
$$

where $g \in \operatorname{Sp}(\mathcal{W}), f \in \mathcal{S}(B), w \in \mathcal{W}$ and $d b$ is a Haar measure on $B^{*}$. It is not difficult to check that $\rho_{\psi}^{B}(h)$ and $M_{B}[g]$ satisfy (2.1.b). We can normalize the measure such that

$$
\left(M_{B}[k] . f\right)(w)=\widetilde{\omega}_{\psi}(k) . f\left(k^{-1} \cdot w\right)
$$

for $k \in K_{B}$ and $f \in \mathcal{S}(B)$. This realization of the Weil representation is known as a generalized lattice model. The cocycle determined by the choice of the operators $M_{B}[g]$ is denoted by $c_{B}\left(g, g^{\prime}\right)$.

2.4. Lattice model. If $B$ happens to be self-dual i.e., $B^{*}=B$, then $\left(M_{B}[g], \mathcal{S}(B)\right)$ is called a lattice model. This model is well-known. We briefly recall the basic facts as follows. Let $A$ be a self-dual lattice in $\mathcal{W}$. Then $\mathcal{S}(A)$ is the space of locally constant, compactly supported functions $f: \mathcal{W} \rightarrow \mathbf{C}$ such that

$$
f(a+w)=\psi\left(\frac{1}{2}\langle\langle w, a\rangle) f(w)\right.
$$

for $a \in A, w \in \mathcal{W}$. Fix a Haar measure $\mu$ of $\mathcal{W}$ such that $\mu(A)=1$. Then we know

$$
\begin{aligned}
\left(\rho_{\psi}^{A}(w, t) . f\right)\left(w^{\prime}\right) & :=\psi\left(\frac{1}{2}\left\langle\left\langle w^{\prime}, w\right\rangle\right\rangle+t\right) f\left(w+w^{\prime}\right) \\
\left(M_{A}[g] . f\right)(w) & :=\int_{A} \psi\left(\frac{1}{2}\langle\langle a, w\rangle) f\left(g^{-1} \cdot(a+w)\right) d \mu(a)\right.
\end{aligned}
$$

where $g \in \operatorname{Sp}(\mathcal{W}), w^{\prime} \in \mathcal{W}$, and $(w, t) \in \mathrm{H}(\mathcal{W})$. 


\section{Admissible splittings of metaplectic covers.}

In Subsection 3.1, we review the basic concept of reductive dual pairs. In Subsection 3.2, the doubling procedure from $[\mathbf{K l}]$ is recalled. Then we introduce a splitting of the metaplectic cover with respect to the generalized lattice model. The main result is Theorem 3.4.

3.1. Reductive dual pairs and admissible splittings. Let $(\mathcal{V}, \mathbf{h})$ (resp. $\left(\mathcal{V}^{\prime}, \mathbf{h}^{\prime}\right)$ ) be an $\epsilon$-hermitian (resp. $\epsilon^{\prime}$-hermitian) space over $D$ such that $\epsilon \epsilon^{\prime}=$ -1 . Define $\mathcal{W}:=\mathcal{V} \otimes_{D} \mathcal{V}^{\prime}$, which will be denoted by $\mathcal{V} \otimes \mathcal{V}^{\prime}$ later for simplicity. Define a skew-symmetric $F$-bilinear from $\langle\langle$,$\rangle on \mathcal{W}$ by

$$
\left\langle\langle,\rangle:=k \operatorname{Trd}_{D / F}\left(\mathbf{h}(,) \otimes \tau \circ \mathbf{h}^{\prime}(,)\right)\right.
$$

where $k:=1$ if $D=F, k:=\frac{1}{2}$ if $D=E$, and $\operatorname{Trd}_{D / F}$ denotes the reduced trace from $D$ to $F$. Recall that $\psi$ is a character of $F$ of conductoral exponent $\lambda_{F}$. Define $\lambda:=\lambda_{F}$ if $D$ is $F$ or an unramified quadratic extension of $F$, $\lambda:=2 \lambda_{F}-1$ if $D$ is a ramified quadratic extension of $F$. Let $\kappa$ (resp. $\left.\kappa^{\prime}\right)$ be the integer to define the dual lattices in $\mathcal{V}$ (resp. $\mathcal{V}^{\prime}$ ) as in (1.3.a). We make the following assumption

$$
\kappa+\kappa^{\prime}=\lambda
$$

throughout the paper.

The pair $\left(U(\mathcal{V}), U\left(\mathcal{V}^{\prime}\right)\right)$ is called a reductive dual pair in $\operatorname{Sp}(\mathcal{W})$. Let $\iota \mathcal{V}^{\prime}$ denote the embedding $U(\mathcal{V}) \rightarrow \operatorname{Sp}(\mathcal{W})$, and $\widetilde{U(\mathcal{V})}$ denote the inverse image of $\iota_{\mathcal{V}^{\prime}}(U(\mathcal{V}))$ in $\widetilde{\operatorname{Sp}(\mathcal{W})}$. The group $\widetilde{U(\mathcal{V})}$ is called a metaplectic cover of $U(\mathcal{V})$. Let $\widehat{U(\mathcal{V})}$ be the two-fold cover of $U(\mathcal{V})$ in $\widetilde{U(\mathcal{V})}$. We know that $\widehat{U(\mathcal{V})}$ is a totally disconnected group. A representation $(\pi, V)$ of $\widetilde{U(\mathcal{V})}$ is called admissible if $\left.\pi\right|_{\mathbf{C}^{\times}}(z)$ is multiplication by $z$ and $\left.\pi\right|_{\widehat{U(\mathcal{V})}}$ is an admissible representation of a totally disconnected group. Restrict the Weil representation of $\widehat{\operatorname{Sp}(\mathcal{W})}$ to $\widetilde{U(\mathcal{V})} \cdot \widetilde{U\left(\mathcal{V}^{\prime}\right)}$ and pull it back via the map $\widetilde{U(\mathcal{V})} \times \widetilde{U\left(\mathcal{V}^{\prime}\right)} \rightarrow \widetilde{U(\mathcal{V})} \cdot \widetilde{U\left(\mathcal{V}^{\prime}\right)}$, then we establish a correspondence between some irreducible admissible representations of $\widetilde{U(\mathcal{V})}$ and $\widetilde{U\left(\mathcal{V}^{\prime}\right)}$. It is proved by R. Howe and J.-L. Waldspurger $[\mathbf{W} \mathbf{p}]$ that the correspondence is one-toone if the residue characteristic of $F$ is odd. This correspondence is called the local theta correspondence.

A function $\beta_{\mathcal{V}^{\prime}}: U(\mathcal{V}) \rightarrow \mathbf{C}^{\times}$is called a splitting of the cocycle $\left.c\right|_{\iota_{\mathcal{V}^{\prime}}(U(\mathcal{V})) \times \iota_{\mathcal{V}^{\prime}}(U(\mathcal{V}))}$ if it satisfies

$$
c\left(\iota \mathcal{V}^{\prime}(g), \iota \mathcal{V}^{\prime}\left(g^{\prime}\right)\right)=\beta_{\mathcal{V}^{\prime}}\left(g g^{\prime}\right) \beta_{\mathcal{V}^{\prime}}(g)^{-1} \beta_{\mathcal{V}^{\prime}}\left(g^{\prime}\right)^{-1}
$$

for any $g, g^{\prime} \in U(\mathcal{V})$. If the extension $\widetilde{U(\mathcal{V})} \rightarrow U(\mathcal{V})$ has a splitting $\beta_{\mathcal{V}^{\prime}}$, then the map $\widetilde{\beta}_{\mathcal{V}^{\prime}}: U(\mathcal{V}) \rightarrow \widetilde{U(\mathcal{V})}$ by $g \mapsto\left(\iota \mathcal{V}^{\prime}(g), \beta_{\mathcal{V}^{\prime}}(g) M\left[\iota \mathcal{V}^{\prime}(g)\right]\right)$ is a group 
homomorphism. A splitting $\beta_{\mathcal{V}^{\prime}}$ is called admissible if $\pi \circ \widetilde{\beta}_{\mathcal{V}^{\prime}}$ is an admissible representation of $U(\mathcal{V})$ whenever $\pi$ is an admissible representation of $\widetilde{U(\mathcal{V})}$. It is known that most of the metaplectic covers split. If we fix an admissible splitting $\beta_{\mathcal{V}^{\prime}}\left(\operatorname{resp} . \beta_{\mathcal{V}}\right)$ of $\widetilde{U(\mathcal{V})}$ (resp. $\widetilde{U\left(\mathcal{V}^{\prime}\right)}$ ), we establish a one-to-one correspondence between some irreducible admissible representations of $U(\mathcal{V})$ and $U\left(\mathcal{V}^{\prime}\right)$. Of course, the correspondence depends on the choices of the splittings. If both $\beta_{1}, \beta_{2}$ are admissible splittings of a cocycle $c\left(\iota \mathcal{V}^{\prime}(g), \iota \mathcal{V}^{\prime}\left(g^{\prime}\right)\right)$, then it is obvious and well-known that $\beta_{1} \beta_{2}^{-1}$ is a character of $U(\mathcal{V})$.

3.2. A doubling procedure. Let $\left(U(\mathcal{V}), U\left(\mathcal{V}^{\prime}\right)\right)$ be a reductive dual pair. An explicit admissible splitting (when it exists) of $\widetilde{U(\mathcal{V})}$ with respect to a Schrödinger model is given by $\mathrm{S}$. Kudla. Here we briefly recall his result. First suppose that the space $\mathcal{V}$ is a direct sum of hyperbolic planes. So we have a complete polarization $\mathcal{V}=X^{\circ}+Y^{\circ}$. Define $X:=X^{\circ} \otimes \mathcal{V}^{\prime}$ and $Y:=$ $Y^{\circ} \otimes \mathcal{V}^{\prime}$. Then $\mathcal{W}=X+Y$ is a complete polarization. Let $\left(M_{Y}[g], \mathcal{S}(Y)\right)$ be the Schrödinger model with respect to $Y$ and a fixed character $\psi$ of $F$. Then an explicit splitting, denoted by $\beta_{\mathcal{V}^{\prime}}^{Y}(g)$ in this paper, is given in $[\mathbf{K l}]$ Theorem 3.1.

If the space $\mathcal{V}$ is not a direct sum of hyperbolic planes, a doubling procedure is required to obtained the splitting as follows (cf. [Kl] Section 4). Let $\overline{\mathcal{V}}$ denote the space $\mathcal{V}$ with the form $\overline{\mathbf{h}}():,=-\mathbf{h}($,$) . Then the space \mathcal{V}+\overline{\mathcal{V}}$ is a direct sum of hyperbolic planes. Let $\mathcal{W}:=\mathcal{V} \otimes \mathcal{V}^{\prime}$ and $\overline{\mathcal{W}}:=\overline{\mathcal{V}} \otimes \mathcal{V}^{\prime}$. We will identify $U(\mathcal{V})$ with the subgroup $U(\mathcal{V}) \times\{1\}$ of $U(\mathcal{V}+\overline{\mathcal{V}})$. We use the same notation $\iota_{\mathcal{V}}^{\prime}$ to denote the inclusions $U(\mathcal{V}) \rightarrow \operatorname{Sp}(\mathcal{W})$ and $U(\mathcal{V}+\overline{\mathcal{V}}) \rightarrow \operatorname{Sp}(\mathcal{W}+\overline{\mathcal{W}})$. Then we have the following maps

$$
U(\mathcal{V}) \hookrightarrow U(\mathcal{V}) \times U(\mathcal{V}) \longrightarrow U(\mathcal{V}+\overline{\mathcal{V}}) \stackrel{\iota^{\prime}{ }^{\prime}}{\longrightarrow} \mathrm{Sp}(\mathcal{W}+\overline{\mathcal{W}})
$$

Define

$$
\begin{aligned}
& X^{\circ}:=\{(v,-v) \mid v \in \mathcal{V}\} \subset \mathcal{V}+\overline{\mathcal{V}}, \\
& Y^{\circ}:=\{(v, v) \mid v \in \mathcal{V}\} \subset \mathcal{V}+\overline{\mathcal{V}} .
\end{aligned}
$$

Then it is clear that the decomposition $\mathcal{V}+\overline{\mathcal{V}}=X^{\circ}+Y^{\circ}$ is a complete polarization. Define $Y:=Y^{\circ} \otimes \mathcal{V}^{\prime}$ and $X:=X^{\circ} \otimes \mathcal{V}^{\prime}$. Then $\mathcal{W}+\overline{\mathcal{W}}=$ $X+Y$ is a complete polarization. Then we can define the Schrödinger model $\left(M_{Y}[g], \mathcal{S}(Y)\right)$ of the Weil representation of $\operatorname{Sp}(\mathcal{W}+\overline{\mathcal{W}})$. We know that there exists the Weil representation $\left(M^{\prime}[g], \mathcal{S}^{\prime}\right)\left(\operatorname{resp} .\left(\bar{M}^{\prime}[\bar{g}], \overline{\mathcal{S}}^{\prime}\right)\right)$ of $\operatorname{Sp}(\mathcal{W})$ (resp. $\operatorname{Sp}(\overline{\mathcal{W}})$ ) such that $\mathcal{S}(Y) \simeq \mathcal{S}^{\prime} \otimes \overline{\mathcal{S}}^{\prime}$ and $M_{Y}[g, \bar{g}] \simeq M^{\prime}[g] \otimes \bar{M}^{\prime}[\bar{g}]$ for $(g, \bar{g}) \in \operatorname{Sp}(\mathcal{W}) \times \operatorname{Sp}(\overline{\mathcal{W}}) \subset \operatorname{Sp}(\mathcal{W}+\overline{\mathcal{W}})$. Let $c^{\prime}$ denote the cocycle associated to $\left(M^{\prime}[g], \mathcal{S}^{\prime}\right)$. Then it is clear that

$$
c_{Y}\left((g, 1),\left(g^{\prime}, 1\right)\right)=c^{\prime}\left(g, g^{\prime}\right)
$$


for $g, g^{\prime} \in \operatorname{Sp}(\mathcal{W})$. Define $\beta_{\mathcal{V}^{\prime}}^{Y}(g):=\beta_{\mathcal{V}^{\prime}}^{Y}(g, 1)$ for $g \in U(\mathcal{V}) \subset U(\mathcal{V}+\overline{\mathcal{V}})$ where $\beta_{\mathcal{V}^{\prime}}^{Y}(g, 1)$ is obtained by the previous paragraph. Then $\beta_{\mathcal{V}^{\prime}}^{Y}(g)$ is an admissible splitting of $\left(M^{\prime}[g], \mathcal{S}^{\prime}\right)$.

Let $L\left(\right.$ resp. $\left.L^{\prime}\right)$ be a good lattice in $\mathcal{V}\left(\right.$ resp. $\left.\mathcal{V}^{\prime}\right)$. Define

$$
B\left(L, L^{\prime}\right):=L^{*} \otimes L^{\prime} \cap L \otimes L^{*} .
$$

Let $\lambda_{F}$ be the integer $\kappa$ to define the dual lattices in $\mathcal{W}$ as in (1.3.a). It is easy to check that $B\left(L, L^{\prime}\right)$ is a good lattice in $\mathcal{W}$, that is, $\varpi_{F} B\left(L, L^{\prime}\right)^{*} \subseteq$ $B\left(L, L^{\prime}\right) \subseteq B\left(L, L^{\prime}\right)^{*}$ by the assumption (3.1.b). It is also easy to verify that $\iota \mathcal{V}^{\prime}\left(U(\mathcal{V})_{L}\right) \subseteq K_{B}$. Let $\bar{L}$ be the corresponding good lattice in $\overline{\mathcal{V}}$. Clearly $B\left(L, L^{\prime}\right)+B\left(\bar{L}, L^{\prime}\right)=B\left(L+\bar{L}, L^{\prime}\right)$ is a good lattice in $\mathcal{W}+\overline{\mathcal{W}}$. Let $B_{1}:=B\left(L, L^{\prime}\right), B_{2}:=B\left(\bar{L}, L^{\prime}\right)$ and $B_{3}:=B\left(L+\bar{L}, L^{\prime}\right)$. It is also clear that we have $\mathcal{S}\left(B_{3}\right) \simeq \mathcal{S}\left(B_{1}\right) \otimes \mathcal{S}\left(B_{2}\right)$ and $M_{B_{3}}[g, \bar{g}]=M_{B_{1}}[g] \otimes M_{B_{2}}[\bar{g}]$ for $g \in \operatorname{Sp}(\mathcal{W})$ and $\bar{g} \in \operatorname{Sp}(\overline{\mathcal{W}})$. In particular,

$$
c_{B_{3}}\left((g, 1),\left(g^{\prime}, 1\right)\right)=c_{B_{1}}\left(g, g^{\prime}\right)
$$

for $g, g^{\prime} \in \operatorname{Sp}(\mathcal{W})$.

3.3. A key proposition. Keep the notation in Subsection 3.2. Let $\mathcal{W}^{\prime}$ be the space $\mathcal{W}$ or $\mathcal{W}+\overline{\mathcal{W}}$ and $B:=B\left(L, L^{\prime}\right)$ or $B\left(L+\bar{L}, L^{\prime}\right)$ depending on whether $\mathcal{V}$ is a direct sum of hyperbolic planes or not. Now we want to compare the actions of $M_{Y}[g]$ and $M_{B}[g]$. Let $\Psi: \mathcal{S}(B) \rightarrow \mathcal{S}(Y)$ be an isomorphism between $\rho_{\psi}^{B}$ and $\rho_{\psi}^{Y}$ i.e., $\rho_{\psi}^{Y}(h)=\Psi^{-1} \circ \rho_{\psi}^{B}(h) \circ \Psi$ for any $h \in \mathrm{H}\left(\mathcal{W}^{\prime}\right)$. Because $M_{Y}[g] \circ \rho_{\psi}^{Y}(h)=\rho_{\psi}^{Y}(g . h) \circ M_{Y}[g]$, we have

$$
\Psi \circ M_{Y}[g] \circ \Psi^{-1} \circ \rho_{\psi}^{B}(h)=\rho_{\psi}^{B}(g . h) \circ \Psi \circ M_{Y}[g] \circ \Psi^{-1} .
$$

By the Stone-Von Neumann theorem, $\Psi^{-1} \circ M_{Y}[g] \circ \Psi$ is a nonzero multiple of $M_{B}[g]$. Hence we define the function $\alpha_{\mathcal{V}^{\prime}}: U(\mathcal{V}) \rightarrow \mathbf{C}^{\times}$by

$$
M_{Y}\left[\iota_{\mathcal{V}^{\prime}}(g)\right] \circ \Psi=\alpha_{\mathcal{V}^{\prime}}(g) \Psi \circ M_{B}\left[\iota \mathcal{V}^{\prime}(g)\right]
$$

for $g \in U(\mathcal{V})$. Since $\Psi$ is unique up to a constant multiple, we see that $\alpha_{\mathcal{V}^{\prime}}$ does not depend on the choice of $\Psi$. Let $c_{Y}\left(\iota \mathcal{V}^{\prime}(g), \iota \mathcal{V}^{\prime}\left(g^{\prime}\right)\right.$ ) (resp. $\left.c_{B}\left(\iota_{\mathcal{V}^{\prime}}(g), \iota \mathcal{V}^{\prime}\left(g^{\prime}\right)\right)\right)$ denote the cocycle on $U(\mathcal{V})$ with respect to the Schrödinger model $\left(M_{Y}[g], \mathcal{S}(Y)\right)$ (resp. the generalized lattice model $\left(M_{B}[g], \mathcal{S}(B)\right)$ ) of the Weil representation of $\operatorname{Sp}\left(\mathcal{W}^{\prime}\right)$. Then it is clear that

$$
c_{Y}\left(\iota_{\mathcal{V}^{\prime}}(g), \iota \mathcal{V}^{\prime}\left(g^{\prime}\right)\right)=\alpha_{\mathcal{V}^{\prime}}(g) \alpha_{\mathcal{V}^{\prime}}\left(g^{\prime}\right) \alpha_{\mathcal{V}^{\prime}}\left(g g^{\prime}\right)^{-1} c_{B}\left(\iota \mathcal{V}^{\prime}(g), \iota \mathcal{V}^{\prime}\left(g^{\prime}\right)\right)
$$

for any $g, g^{\prime} \in U(\mathcal{V})$. Therefore $\alpha_{\mathcal{V}^{\prime}}(g) \beta_{\mathcal{V}^{\prime}}^{Y}(g)$ is a splitting of the cocycle $c_{B}\left(\iota_{\mathcal{V}^{\prime}}(g), \iota_{\mathcal{V}^{\prime}}\left(g^{\prime}\right)\right)$. And it is also clear that $\alpha_{\mathcal{V}^{\prime}}(g) \beta_{\mathcal{V}^{\prime}}^{Y}(g)$ is an admissible splitting since $\beta_{\mathcal{V}^{\prime}}^{Y}(g)$ is admissible. From (2.3.e), we know that $c_{B}\left(g, g^{\prime}\right)=1$ if both $g, g^{\prime} \in K_{B}$. Therefore $\left.\alpha_{\mathcal{V}^{\prime}} \beta_{\mathcal{V}^{\prime}}^{Y}\right|_{U(\mathcal{V})_{L}}$ is a character of $U(\mathcal{V})_{L}$ because $\iota \mathcal{V}^{\prime}\left(U(\mathcal{V})_{L}\right) \subseteq K_{B}$.

Now we define a character $\zeta \mathcal{V}^{\prime}$ of $U(\mathcal{V})_{L}$ as follows. 
(i) If $D=F$, let $\zeta_{\mathcal{V}^{\prime}}$ be the trivial character of $U(\mathcal{V})_{L}$.

(ii) Suppose that $D$ is an unramified extension of $F$. If one of $1, \mathbf{l}^{*}$ is trivial, let $\zeta_{\mathcal{V}^{\prime}}$ be the trivial character of $U(\mathcal{V})_{L}$. Now suppose that both $\mathbf{l}$ and $\mathbf{l}^{*}$ are not trivial. Then $S\left(U(\mathbf{l}) \times U\left(\mathbf{l}^{*}\right)\right) /\left(S U(\mathbf{l}) \times S U\left(\mathbf{l}^{*}\right)\right)$ is a finite cyclic group order $q+1$. Let $\zeta_{0}$ be the unique character of $S\left(U(\mathbf{l}) \times U\left(\mathbf{l}^{*}\right)\right) /\left(S U(\mathbf{l}) \times S U\left(\mathbf{l}^{*}\right)\right)$ of order two. Let $\zeta_{\mathcal{V}^{\prime}}$ be the character of $U(\mathcal{V})_{L}$ lifted from the character of $U(\mathcal{V})_{L} /\left[U(\mathcal{V})_{L}, U(\mathcal{V})_{L}\right]$ extended trivially from $\zeta_{0}$ by (1.4.a) if $\mathcal{V}^{\prime}$ is odd-dimensional; let $\zeta_{\mathcal{V}^{\prime}}$ be the trivial character of $U(\mathcal{V})_{L}$ if $\mathcal{V}^{\prime}$ is even-dimensional.

(iii) Suppose that $D$ is a ramified extension of $F$. Then one of $1, \mathbf{l}^{*}$ is a quadratic space, and the other is a symplectic space. Let $\mathbf{l}^{\prime}$ denote the quadratic space. If the dimension of $\mathbf{l}^{\prime}$ is zero or one, let $\zeta_{\mathcal{V}^{\prime}}$ be the trivial character of $U(\mathcal{V})_{L}$. If the dimension of $\mathbf{l}^{\prime}$ is greater than one, then we know that $S U\left(\mathbf{l}^{\prime}\right) /\left[U\left(\mathbf{l}^{\prime}\right), U\left(\mathbf{l}^{\prime}\right)\right] \simeq \mathbf{Z} / 2 \mathbf{Z}$ and let $\zeta_{0}$ be the nontrivial character of $S U\left(\mathbf{l}^{\prime}\right) /\left[U\left(\mathbf{l}^{\prime}\right), U\left(\mathbf{l}^{\prime}\right)\right]$. Then $\zeta_{0}$ can be extended trivially via (1.4.a) to be a character (still denoted by $\zeta_{0}$ ) of $U(\mathcal{V})_{L} /\left[U(\mathcal{V})_{L}, U(\mathcal{V})_{L}\right]$. If $\mathcal{V}^{\prime}$ is odd-dimensional, let $\zeta_{\mathcal{V}^{\prime}}$ be the character of $U(\mathcal{V})_{L}$ lifted from $\zeta_{0}$ of the group $U(\mathcal{V})_{L} /\left[U(\mathcal{V})_{L}, U(\mathcal{V})_{L}\right]$; if $\mathcal{V}^{\prime}$ is even-dimensional, let $\zeta_{\mathcal{V}^{\prime}}$ be the trivial character of $U(\mathcal{V})_{L}$.

Although the notation of the character $\zeta \mathcal{V}^{\prime}$ suggests that it depends on $\mathcal{V}^{\prime}$, in fact it only depends on the parity of the dimension of $\mathcal{V}^{\prime}$.

Proposition. Keep the notation as above. Then the restrictions to $S U(\mathcal{V})_{L}$ of $\alpha_{\mathcal{V}^{\prime}} \beta_{\mathcal{V}^{\prime}}^{Y}$ and $\zeta_{\mathcal{V}^{\prime}}$ are equal.

Since both $\alpha \mathcal{V}^{\prime} \beta_{\mathcal{V}^{\prime}}^{Y}$ and $\zeta_{\mathcal{V}^{\prime}}$ are characters of $U(\mathcal{V})_{L}$, the restrictions $\left.\alpha \mathcal{V}^{\prime} \beta_{\mathcal{V}^{\prime}}^{Y}\right|_{\left[U(\mathcal{V})_{L}, U(\mathcal{V})_{L}\right]}$ and $\left.\zeta_{\mathcal{V}^{\prime}}\right|_{\left[U(\mathcal{V})_{L}, U(\mathcal{V})_{L}\right]}$ are trivial. So to prove this proposition we only need to check $\alpha_{\mathcal{V}^{\prime}}(g) \beta_{\mathcal{V}^{\prime}}^{Y}(g)=\zeta_{\mathcal{V}^{\prime}}(g)$ where $g$ runs over a set of representatives of $\left[U(\mathcal{V})_{L}, U(\mathcal{V})_{L}\right]$-cosets in $S U(\mathcal{V})_{L}$. So the result in Subsection 1.4 plays a role in the proof of this proposition. We also remark here that $\beta_{\mathcal{V}^{\prime}}^{Y}$ depends on a choice of a character $\chi$ of $E^{\times}$if $U(\mathcal{V})$ is a unitary group. For different $\chi, \beta_{\mathcal{V}^{\prime}}^{Y}$ may be different by a character $\zeta$ of $U(\mathcal{V})$ (or $U(\mathcal{V}+\overline{\mathcal{V}})$ ). Such a character $\zeta$ must factor through the determinant map. Therefore we only need to prove the proposition for a proper chosen character $\chi$ if $U(\mathcal{V})$ is a unitary group. The remaining computation for the proposition is postponed to Sections 6-9.

3.4. A nice splitting with respect to a generalized lattice model. The following theorem is our first main result.

Theorem. Suppose that the extension $\widetilde{U(\mathcal{V})} \rightarrow U(\mathcal{V})$ splits. Let $L$ be a good lattice in $\mathcal{V}$ and $L^{\prime}$ be a good lattice in $\mathcal{V}^{\prime}$. Let $B$ be the lattice $B\left(L, L^{\prime}\right)$ or $B\left(L+\bar{L}, L^{\prime}\right)$ depending on whether $\mathcal{V}$ is a direct sum of hyperbolic planes or not. Let $\zeta_{\mathcal{V}^{\prime}}$ be the character of $U(\mathcal{V})_{L}$ defined in Subsection 3.3. 
Then there exists an admissible splitting $\beta_{\mathcal{V}^{\prime}}^{L}: U(\mathcal{V}) \rightarrow \mathbf{C}^{\times}$of the cocycle $c_{B}\left(\iota \mathcal{V}^{\prime}(g), \iota \mathcal{V}^{\prime}\left(g^{\prime}\right)\right)$ such that $\beta_{\mathcal{V}^{\prime}}^{L}(g)=\zeta \mathcal{V}^{\prime}(g)$ for any $g \in U(\mathcal{V})_{L}$. Moreover the splitting $\beta_{\mathcal{V}^{\prime}}^{L}$ is unique except the following two cases: $(1) U(\mathcal{V})$ is an isotropic orthogonal group, or (2) $E$ is a ramified quadratic extension and the orthogonal space in $\mathbf{l}_{1} \mathbf{l}^{*}$ is trivial. For the exceptional cases, there are two such splittings.

Proof. Define

$$
K:= \begin{cases}\{1\}, & \text { if } U(\mathcal{V}) \text { is a symplectic group; } \\ \{ \pm 1\}, & \text { if } U(\mathcal{V}) \text { is an orthogonal group; } \\ E^{(1)}, & \text { if } U(\mathcal{V}) \text { is a unitary group. }\end{cases}
$$

Let det: $U(\mathcal{V}) \rightarrow K$ be the determinant map. By Proposition 3.3, the character $\alpha_{\mathcal{V}^{\prime}} \beta_{\mathcal{V}^{\prime}}^{Y} \zeta_{\mathcal{V}^{\prime}}=\alpha_{\mathcal{V}^{\prime}} \beta_{\mathcal{V}^{\prime}}^{Y} \zeta_{\mathcal{V}^{\prime}}^{-1}$ is trivial when restricted to $S U(\mathcal{V})_{L}$. Therefore, the character $\alpha_{\mathcal{V}^{\prime}} \beta_{\mathcal{V}^{\prime}}^{Y} \zeta_{\mathcal{V}^{\prime}}$ of $U(\mathcal{V})_{L}$ factors through the determinant map i.e., $\alpha \mathcal{V}^{\prime} \beta_{\mathcal{V}^{\prime}}^{Y} \zeta_{\mathcal{V}^{\prime}}=\xi^{\prime} \circ\left(\left.\operatorname{det}\right|_{U(\mathcal{V})_{L}}\right)$ for some character $\xi^{\prime}$ of $\operatorname{det}\left(U(\mathcal{V})_{L}\right)$. It is obvious that either $\operatorname{det}\left(U(\mathcal{V})_{L}\right)=K$ or $\operatorname{det}\left(U(\mathcal{V})_{L}\right)$ is a subgroup of index two of $K$. In both cases, it is clear that a character of $\operatorname{det}\left(U(\mathcal{V})_{L}\right)$ is the restriction of a character of $K$. So we may assume that $\xi^{\prime}$ is a character of $K=\operatorname{det}(U(\mathcal{V}))$. Hence $\alpha_{\mathcal{V}^{\prime}} \beta_{\mathcal{V}^{\prime}}^{Y} \zeta_{\mathcal{V}^{\prime}}$ is the restriction of the character $\xi^{\prime} \circ \operatorname{det}$ of $U(\mathcal{V})$ i.e., $\alpha_{\mathcal{V}^{\prime}} \beta_{\mathcal{V}^{\prime}}^{Y} \zeta_{\mathcal{V}^{\prime}}=\left.\left(\xi^{\prime}\right.$ o det $)\right|_{U(\mathcal{V})_{L}}$. Let $\xi$ denote the character $\xi^{\prime} \circ \operatorname{det}$ of $U(\mathcal{V})$. Define

$$
\beta_{\mathcal{V}^{\prime}}^{L}(g):=\xi(g)^{-1} \alpha_{\mathcal{V}^{\prime}}(g) \beta_{\mathcal{V}^{\prime}}^{Y}(g)
$$

for any $g \in U(\mathcal{V})$. Since $\alpha_{\mathcal{V}^{\prime}}(g) \beta_{\mathcal{V}^{\prime}}^{Y}(g)$ is an admissible splitting of $c_{B}\left(\iota_{\mathcal{V}^{\prime}}(g)\right.$, $\left.\iota \mathcal{V}^{\prime}\left(g^{\prime}\right)\right)$, so is $\beta_{\mathcal{V}^{\prime}}^{L}(g)$. And it is clear that $\left.\beta_{\mathcal{V}^{\prime}}^{L}\right|_{U(\mathcal{V})_{L}}$ is equal to $\zeta_{\mathcal{V}^{\prime}}$.

Next we want to prove that the admissible splitting satisfying the condition in Theorem 3.4 is almost unique. Suppose that $\beta$ is another admissible splitting of $c_{B}\left(\iota_{\mathcal{V}^{\prime}}(g), \iota_{\mathcal{V}^{\prime}}\left(g^{\prime}\right)\right)$ and $\left.\beta\right|_{U(\mathcal{V})_{L}}=\zeta_{\mathcal{V}^{\prime}}$. Then $\beta^{-1} \beta_{\mathcal{V}^{\prime}}^{L}$ is a character of $U(\mathcal{V})$ whose restriction to $U(\mathcal{V})_{L}$ is trivial. If $\mathcal{V}$ is anisotropic, then $U(\mathcal{V})=U(\mathcal{V})_{L}$. So $\beta^{-1} \beta_{\mathcal{V}^{\prime}}^{L}$ is the trivial character of $U(\mathcal{V})$. Hence $\beta=\beta_{\mathcal{V}^{\prime}}^{L}$. Next suppose that $\mathcal{V}$ is isotropic and $U(\mathcal{V})$ is not an orthogonal group. It is known that $[U(\mathcal{V}), U(\mathcal{V})]=S U(\mathcal{V})$. So $\beta^{-1} \beta_{\mathcal{V}^{\prime}}^{L}=\phi \circ \operatorname{det}$ for some character $\phi$ of $K$. If we also assume that we are not in case (2) of the theorem, then we know that $\operatorname{det}\left(U(\mathcal{V})_{L}\right)=K$, so $\phi$ must be trivial. So $\beta^{-1} \beta_{\mathcal{V}^{\prime}}^{L}$ is a trivial character of $U(\mathcal{V})$ i.e., $\beta=\beta_{\mathcal{V}^{\prime}}^{L}$. If we are in case (2) of the theorem, then $\operatorname{det}\left(U(\mathcal{V})_{L}\right)$ is a subgroup of $K$ of index 2 i.e., $\operatorname{det}\left(U(\mathcal{V})_{L}\right)$ are the numbers $t \in E^{(1)}$ such that $t \equiv 1 \bmod \mathfrak{p}_{E}$. There are two characters of $K$ whose restriction to $\operatorname{det}\left(U(\mathcal{V})_{L}\right)$ is trivial, namely, the trivial character and the unique character of order two. So we have two splittings in this case. Now we consider the remaining case i.e., $\mathcal{V}$ is an isotropic quadratic space. In this case, it is well-known that $U(\mathcal{V}) /[U(\mathcal{V}), U(\mathcal{V})] \simeq(\mathbf{Z} / 2 \mathbf{Z})^{3}$. 
Suppose that $\left\{g_{1}, g_{2}, g_{3}\right\}$ is a set of representatives of the cosets which generate $U(\mathcal{V}) /[U(\mathcal{V}), U(\mathcal{V})]$. It is easy to see from Subsection 1.4 that we can choose the set $\left\{g_{1}, g_{2}, g_{3}\right\}$ such that two of the elements in the set are also in $U(\mathcal{V})_{L}$. Hence there are exactly two characters of $U(\mathcal{V})$ whose restrictions to $U(\mathcal{V})_{L}$ are trivial. So there are two admissible splittings of $c_{B}\left(\iota_{\mathcal{V}^{\prime}}(g), \iota_{\mathcal{V}^{\prime}}\left(g^{\prime}\right)\right)$ whose restrictions to $U(\mathcal{V})_{L}$ are equal to $\zeta_{\mathcal{V}^{\prime}}$.

3.5. Remark. From Theorem 3.4, we see that the map $\widetilde{\beta}_{\mathcal{V}^{\prime}}^{L}: U(\mathcal{V}) \rightarrow \widetilde{U(\mathcal{V})}$ defined by

$$
\widetilde{\beta}_{\mathcal{V}^{\prime}}^{L}(g):=\left(\iota \mathcal{V}^{\prime}(g), \beta_{\mathcal{V}^{\prime}}^{L}(g) M_{B}\left[\iota \mathcal{V}^{\prime}(g)\right]\right)
$$

is a group homomorphism. Regard $\left(\omega_{\psi}, \mathcal{S}(B)\right)$ as a representation of $U(\mathcal{V})$ via $\widetilde{\beta}_{\mathcal{V}^{\prime}}^{L}$. Then by (2.3.e) and the fact $\left.\beta_{\mathcal{V}^{\prime}}^{L}\right|_{U(\mathcal{V})_{L}}=\zeta \mathcal{V}^{\prime}$, we see that

$$
\left(\omega_{\psi}\left(\iota \mathcal{V}^{\prime}(k)\right) \cdot f\right)(w)=\zeta \mathcal{V}^{\prime}(k) \widetilde{\omega}_{\psi}\left(\iota \mathcal{V}^{\prime}(k)\right) \cdot f\left(\iota \mathcal{V}^{\prime}(k)^{-1} \cdot w\right)
$$

for $w \in \mathcal{W}, k \in U(\mathcal{V})_{L}$ and $f \in \mathcal{S}(B)$. Note that $\zeta_{\mathcal{V}^{\prime}}$ is either trivial or of order two. Hence (3.5.b) is very convenient to study local theta correspondence via the theory of minimal $K$-types. In fact, it is a fundamental ingredient of the study of the relation between the theta correspondence of a $p$-adic reductive dual pair and the theta correspondence of a finite reductive dual pair. More details can be found in [Pn2].

Although the lattice $B$ depends on the choice of the lattice $L^{\prime}$ of $\mathcal{V}^{\prime}$, it turns out that the splitting $\beta_{\mathcal{V}^{\prime}}^{L}$ does not at least if we exclude the two exceptional cases in Theorem 3.4. This justifies the use of our notation " $\beta_{\mathcal{V}}^{L}$ " instead of " $\beta_{\mathcal{V}^{\prime}}^{B}$ ".

3.6. Tensor product of the Weil representations. Suppose that $\mathcal{W}=$ $\bigoplus_{i} \mathcal{W}_{i}$ where $\mathcal{W}_{i}$ are nondegenerate symplectic subspaces of $\mathcal{W}$. Suppose that a maximal totally isotropic subspace $Y$ is compatible with the decomposition i.e., $Y=\bigoplus_{i} Y_{i}$ where $Y_{i}:=Y \cap \mathcal{W}_{i}$. Then it is obvious that $\mathcal{S}(Y) \simeq \bigotimes_{i} \mathcal{S}\left(Y_{i}\right)$ and $M_{Y}[g] \simeq \bigotimes_{i} M_{Y_{i}}\left[g_{i}\right]$ where $g:=\prod_{i} g_{i} \in \prod_{i} \operatorname{Sp}\left(\mathcal{W}_{i}\right) \subseteq$ $\operatorname{Sp}(\mathcal{W})$ and $\left(M_{Y_{i}}\left[g_{i}\right], \mathcal{S}\left(Y_{i}\right)\right)$ is a Schrödinger model of the Weil representation of $\operatorname{Sp}\left(\mathcal{W}_{i}\right)$. Similarly, if a good lattice $B$ in $\mathcal{W}$ is compatible with the decomposition $\mathcal{W}=\bigoplus_{i} \mathcal{W}_{i}$ i.e., $B=\bigoplus_{i} B_{i}$ where $B_{i}:=B \cap \mathcal{W}_{i}$, then $\mathcal{S}(B) \simeq \bigotimes_{i} \mathcal{S}\left(B_{i}\right)$ and $M_{B}[g]=\bigotimes_{i} M_{B_{i}}\left[g_{i}\right]$ where $\left(M_{B_{i}}\left[g_{i}\right], \mathcal{S}\left(B_{i}\right)\right)$ is a generalized lattice model of the Weil representation of $\operatorname{Sp}\left(\mathcal{W}_{i}\right)$.

Suppose that $\mathcal{V}=\bigoplus_{i} \mathcal{V}_{i}$ is an $L$-admissible decomposition of nondegenerate subspaces and $Y^{\circ}$ is an maximal totally isotropic of $\mathcal{V}$ such that $Y^{\circ}=\bigoplus_{i} Y_{i}^{\circ}$ where $Y_{i}^{\circ}:=Y^{\circ} \cap \mathcal{V}_{i}$. Let $Y_{i}:=Y_{i}^{\circ} \otimes \mathcal{V}^{\prime}, L_{i}:=L \cap \mathcal{V}_{i}$ and $B_{i}:=B\left(L_{i}, L^{\prime}\right)$. If $g:=\prod_{i} g_{i} \in \prod_{i} U\left(\mathcal{V}_{i}\right) \subseteq U(\mathcal{V})$, then it is clear from the previous paragraph that

$$
\beta_{\mathcal{V}^{\prime}}^{Y}(g)=\prod_{i} \beta_{\mathcal{V}^{\prime}}^{Y_{i}}\left(g_{i}\right) \quad \text { and } \quad \alpha_{\mathcal{V}^{\prime}}(g)=\prod_{i} \alpha_{\mathcal{V}_{i}^{\prime}}\left(g_{i}\right)
$$


Suppose that $\mathcal{V}^{\prime}=\bigoplus_{i} \mathcal{V}_{i}^{\prime}$ is a decomposition of nondegenerate subspaces. Let $Y_{i}:=Y^{\circ} \otimes \mathcal{V}_{i}^{\prime}$. Then it is clear that

$$
c_{Y}\left(\iota \mathcal{V}^{\prime}(g), \iota \mathcal{V}^{\prime}\left(g^{\prime}\right)\right)=\prod_{i} c_{Y_{i}}\left(\iota \mathcal{V}_{i}^{\prime}(g), \iota \mathcal{V}_{i}^{\prime}\left(g^{\prime}\right)\right)
$$

for $g, g^{\prime} \in U(\mathcal{V})$. If $U(\mathcal{V})$ is an orthogonal group or a symplectic group, it is clear that

$$
\beta_{\mathcal{V}^{\prime}}^{Y}(g)=\prod_{i} \beta_{\mathcal{V}_{i}^{\prime}}^{Y_{i}}(g)
$$

If $U(\mathcal{V})$ is a unitary group, the definition of $\beta_{\mathcal{V}^{\prime}}^{Y}(g)$ depends on a choice of a character $\chi$ of $E^{\times}$such that $\left.\chi\right|_{F^{\times}}=\epsilon_{E / F}^{m}$ where $m=\operatorname{dim}\left(\mathcal{V}^{\prime}\right)$ and $\epsilon_{E / F}$ denotes the quadratic character of $F^{\times}$with respect to the extension $E$ of $F$. If we choose characters $\chi_{i}$ of $E^{\times}$such that $\left.\chi_{i}\right|_{F^{\times}}=\epsilon_{E / F}^{m_{i}}$ where $m_{i}=\operatorname{dim}\left(\mathcal{V}_{i}^{\prime}\right)$ and let $\chi:=\bigotimes_{i} \chi_{i}$, then clearly $\left.\chi\right|_{F^{\times}}=\epsilon_{E / F^{m}}^{m}$. Therefore (3.6.c) is also true for unitary groups if we define $\beta_{\mathcal{V}_{i}^{\prime}}^{Y_{i}}\left(\right.$ resp. $\left.\beta_{\mathcal{V}^{\prime}}^{Y}\right)$ with respect to the character $\chi_{i}$ (resp. $\chi$ ). If we also assume that the decomposition $\mathcal{V}^{\prime}=\bigoplus_{i} \mathcal{V}_{i}^{\prime}$ is $L^{\prime}$ admissible, then clearly

$$
c_{B}\left(\iota \mathcal{V}^{\prime}(g), \iota \mathcal{V}^{\prime}\left(g^{\prime}\right)\right)=\prod_{i} c_{B_{i}}\left(\iota \mathcal{V}_{i}^{\prime}(g), \iota \mathcal{V}_{i}^{\prime}\left(g^{\prime}\right)\right)
$$

where $B_{i}:=B\left(L, L_{i}^{\prime}\right)$ and $L_{i}^{\prime}:=L^{\prime} \cap \mathcal{V}_{i}$. Moreover, it is clear that $\alpha_{\mathcal{V}^{\prime}}(g)=$ $\prod_{i} \alpha_{\mathcal{V}_{i}^{\prime}}(g)$. Hence,

$$
\alpha_{\mathcal{V}^{\prime}}(g) \beta_{\mathcal{V}^{\prime}}^{Y}(g)=\prod_{i} \alpha_{\mathcal{V}_{i}^{\prime}}(g) \beta_{\mathcal{V}_{i}^{\prime}}^{Y_{i}}(g)
$$

\section{Isomorphisms between various models.}

In this section, we discuss isomorphisms between various models of the Weil representation introduced in Section 2. These isomorphisms will be used in Sections 6-9.

4.1. Schrödinger models and lattice models. Let $\mathcal{W}$ be a symplectic space over $F$ and $\mathcal{W}=X+Y$ be a complete polarization. Let $L_{X}$ be a lattice in $X$. Let $L_{Y}$ be the dual lattice of $L_{X}$ in $Y$. Then $A:=L_{X}+$ $L_{Y}$ is a self-dual lattice in $\mathcal{W}$. Let $\left(\rho_{\psi}^{Y}, \mathcal{S}(Y)\right)\left(\operatorname{resp} .\left(\rho_{\psi}^{A}, \mathcal{S}(A)\right)\right)$ be the Schrödinger model (resp. lattice model) of the irreducible representation of the Heisenberg group $\mathrm{H}(\mathcal{W})$ with respect to a given nontrivial character $\psi$ of $F$. Choose a Haar measure $\mu_{X}$ on $X$. For $f \in \mathcal{S}(A)$, define a function $\Xi . f: Y \rightarrow \mathbf{C}$ by

$$
(\Xi . f)(y):=\int_{X} \psi\left(\frac{1}{2}\langle\langle x, y\rangle) f(x+y) d \mu_{X}(x) .\right.
$$


Since $f$ is compactly supported, $\Xi . f$ is well-defined. Clearly $\Xi . f$ is locally constant because $f$ is. It is also not difficult to check that $\Xi$. $f$ is compactly supported. Hence $\Xi . f \in \mathcal{S}(Y)$.

Lemma. The map $\Xi$ is an isomorphism of vector spaces and the following diagram

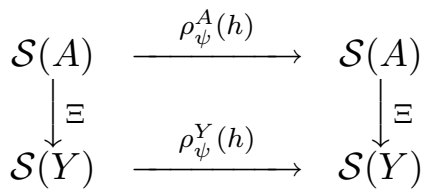

commutes for any $h \in \mathrm{H}(\mathcal{W})$.

Proof. We may only consider $h:=x+y \in \mathcal{W}$ where $x \in X, y \in Y$. Let $f \in \mathcal{S}(A)$. Then

$$
\begin{aligned}
& \left(\rho_{\psi}^{Y}(x+y)(\Xi . f)\right)\left(y^{\prime}\right) \\
& =\psi\left(\left\langle\frac{y}{2}+y^{\prime}, x\right\rangle\right)(\Xi . f)\left(y+y^{\prime}\right) \\
& =\psi\left(\left\langle\frac{y}{2}+y^{\prime}, x\right\rangle\right) \int_{X} \psi\left(\frac{1}{2}\left\langle\left\langle x^{\prime}, y+y^{\prime}\right\rangle\right) f\left(x^{\prime}+y+y^{\prime}\right) d \mu_{X}\left(x^{\prime}\right) .\right.
\end{aligned}
$$

On the other hand,

$$
\begin{aligned}
& \left(\Xi\left(\rho_{\psi}^{A}(x+y) \cdot f\right)\right)\left(y^{\prime}\right) \\
& =\int_{X} \psi\left(\frac{1}{2}\left\langle x^{\prime}, y^{\prime}\right\rangle\right)\left(\rho_{\psi}^{A}(x+y) \cdot f\right)\left(x^{\prime}+y^{\prime}\right) d \mu_{X}\left(x^{\prime}\right) \\
& =\int_{X} \psi\left(\frac { 1 } { 2 } \langle \langle x ^ { \prime } , y ^ { \prime } \rangle ) \psi \left(\frac{1}{2}\left\langle\left\langle x^{\prime}+y^{\prime}, x+y\right\rangle\right) f\left(x+y+x^{\prime}+y^{\prime}\right) d \mu_{X}\left(x^{\prime}\right)\right.\right. \\
& =\psi\left(\left\langle\frac{y}{2}+y^{\prime}, x\right\rangle\right) \int_{X} \psi\left(\frac{1}{2}\left\langle\left\langle x^{\prime}+x, y+y^{\prime}\right\rangle\right) f\left(x^{\prime}+x+y+y^{\prime}\right) d \mu_{X}\left(x^{\prime}\right) .\right.
\end{aligned}
$$

Hence, the diagram commutes. Because the representations $\rho_{\psi}^{Y}$ and $\rho_{\psi}^{A}$ are irreducible and clearly $\Xi$ is not trivial, $\Xi$ must be an isomorphism of vector spaces.

\subsection{Two models of the Weil representation of a finite symplectic} group. Let $(\mathbf{w},\langle\langle\rangle$,$) be a symplectic space over a finite field \mathbf{f}$ and $\bar{\psi}$ a nontrivial character of $\mathbf{f}$. Let $\bar{\psi}_{0}$ be the character of $\mathbf{f}$ defined by $\bar{\psi}_{0}(t)=$ $\bar{\psi}(t / 2)$ for $t \in \mathbf{f}$. Let $\mathbf{w}=\mathbf{x}+\mathbf{y}$ be a complete polarization. Let $S_{1}(\mathbf{y})$ be the space of complex valued functions on $\mathbf{y}$. For $g \in \operatorname{Sp}(\mathbf{w})$, let $x(g)$ and $j(g)$ be as defined in $[\mathbf{R R}]$ and $M_{1}[g] \in \operatorname{Aut}\left(S_{1}(\mathbf{y})\right)$ be the $M_{Y}[g]$ defined in Subsection 2.2. Here the measure $\mu_{g}$ on the space $\mathbf{x} / \operatorname{Ker}(c)$ is chosen such that $\mu_{g}(\mathbf{x} / \operatorname{Ker}(c))=q^{j(g) / 2}$ where $q=\#(\mathbf{f})$. Let

$$
m(g):=\gamma_{\mathbf{f}}\left(x(g), \bar{\psi}_{0}\right)^{-1} \gamma_{\mathbf{f}}\left(\bar{\psi}_{0}\right)^{-j(g)}
$$


be as defined in $[\mathbf{R R}]$ p. 360 . It is known that the map $g \mapsto m(g) M_{1}[g]$ is an ordinary representation of the finite group $\operatorname{Sp}(\mathbf{w})$ from $[\mathbf{R R}]$ Corollary 5.7.

We want to introduce another realization of the Weil representation of the finite symplectic group $\operatorname{Sp}(\mathbf{w})$. Let $S_{2}(\mathbf{y})$ be the space of functions $f: \mathbf{w} \rightarrow \mathbf{C}$ such that

$$
f(a+w)=\bar{\psi}\left(\frac{1}{2}\langle\langle w, a\rangle) f(w)\right.
$$

for $w \in \mathbf{w}, a \in \mathbf{x}$. Let $\mu_{\mathbf{x}}$ be the Haar measure in $\mathbf{x}$ such that $\mu_{\mathbf{x}}(\mathbf{x})=1$. Define

$$
\left(M_{2}[g] . f\right)(w):=\int_{\mathbf{x}} \psi\left(\frac{1}{2}\langle\langle a, w\rangle\rangle\right) f\left(g^{-1} \cdot(a+w)\right) d \mu_{\mathbf{x}}(a) .
$$

Let $\bar{\Upsilon}: S_{2}(\mathbf{y}) \rightarrow S_{1}(\mathbf{y})$ be the isomorphism of vector spaces by

$$
\bar{\Upsilon} . f:=\left.f\right|_{\mathbf{y}}
$$

for $f \in S_{2}(\mathbf{y})$.

Lemma. The following diagram

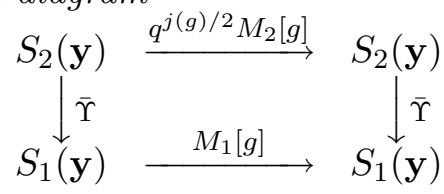

commutes for any $g \in \operatorname{Sp}(\mathbf{w})$.

Proof. We can define the representations $\rho_{1}$ (resp. $\rho_{2}$ ) of $\mathrm{H}(\mathbf{w})$ on the space $S_{1}(\mathbf{y})$ (resp. $S_{2}(\mathbf{y})$ ) and check that the map $\bar{\Upsilon}$ intertwines these two representations. By the Stone-Von Neumann theorem, we know that there exists a function $\alpha: \operatorname{Sp}(\mathbf{w}) \rightarrow \mathbf{C}^{\times}$such that

$$
M_{1}[g] \circ \bar{\Upsilon}=\bar{\Upsilon} \circ \alpha(g) M_{2}[g]
$$

for $g \in \operatorname{Sp}(\mathbf{w})$. So we just need to compute $\alpha(g)$. Let $f_{1} \in S_{1}(\mathbf{y})$ be the characteristic function of $\{0\}$ and $f_{2} \in S_{2}(\mathbf{y})$ be the characteristic function of $\mathbf{x}$. It is clear that $\bar{\Upsilon} \cdot f_{2}=f_{1}$. We have

$$
\begin{aligned}
\left(\bar{\Upsilon} \circ M_{2}[g] f_{2}\right)(0) & \left.=\left(M_{2}[g] f_{2}\right)(0)=\int_{\mathbf{x}} \bar{\psi}(\langle a, 0\rangle\rangle\right) f_{2}\left(g^{-1}(a+0)\right) d \mu_{\mathbf{x}}(a) \\
& =\mu_{\mathbf{x}}(g \cdot \mathbf{x} \cap \mathbf{x})=q^{-j(g)} .
\end{aligned}
$$

On the other hand, we have

$$
\begin{aligned}
\left(M_{1}[g] . f_{1}\right)(0) & =\int_{\mathbf{x} / \operatorname{Ker}(c)} \bar{\psi}\left(\left\langle c^{*} \cdot x, d^{*} \cdot x\right\rangle\right) f_{1}\left(-c^{*} \cdot x\right) d \mu_{g}(x) \\
& =\mu_{g}(\{0\})=q^{j(g) / 2}\left(q^{j(g)}\right)^{-1} \\
& =q^{-j(g) / 2} .
\end{aligned}
$$


Hence, $\alpha(g)=q^{j(g) / 2}$.

Therefore, $\left(m(g) q^{j(g) / 2} M_{2}[g], S_{2}(\mathbf{y})\right)$ is an ordinary representation of the finite symplectic group $\operatorname{Sp}(\mathbf{w})$.

4.3. Generalized lattice model revisited. Let $B$ be a good lattice in $\mathcal{W}$ and $A$ be a self-dual lattice such that $B \subseteq A \subseteq B^{*}$. Let $\mathbf{b}^{*}:=B^{*} / B$ and $\Pi_{B^{*}}$ denote the map $B^{*} \rightarrow \mathbf{b}^{*}$. We know that $\mathbf{b}^{*}$ is a symplectic space over $\mathbf{f}_{F}$ and $\Pi_{B^{*}}(A)$ is a maximal totally isotropic subspace of $\mathbf{b}^{*}$. Let $K_{B}$ and $K_{B}^{\prime}$ be as defined in Subsection 2.3. We know that $K_{B} / K_{B}^{\prime} \simeq \operatorname{Sp}\left(\mathbf{b}^{*}\right)$. Let $S\left(B^{*}\right)$ be the space of locally constant, functions $f: B^{*} \rightarrow \mathbf{C}$ such that

$$
f(a+b)=\psi\left(\frac{1}{2}\langle b, a\rangle\right) f(b)
$$

for $a \in A$ and $b \in B^{*}$. For $g \in K_{B}$, let $\bar{g}$ denote its image in $\operatorname{Sp}\left(\mathbf{b}^{*}\right)$. Let $M_{A}[g]$ be the $M[g]$ defined in [MVW] p. 42. If $g \in K_{B}$ and $f \in S\left(B^{*}\right)$, then clearly $M_{A}[g] . f \in S\left(B^{*}\right)$. Moreover, $M_{A}[g]$ acts trivially on $S\left(B^{*}\right)$ if $g \in K_{B}^{\prime}$. Let $\mathbf{w}:=\mathbf{b}^{*}$ and $\mathbf{y}:=\Pi_{B^{*}}(A)$. Hence, $\mathbf{y}$ is a maximal totally isotropic subspace of $\mathbf{w}$. Clearly, we have an isomorphism $\Upsilon: S\left(B^{*}\right) \rightarrow S_{2}(\mathbf{y})$ such that the diagram

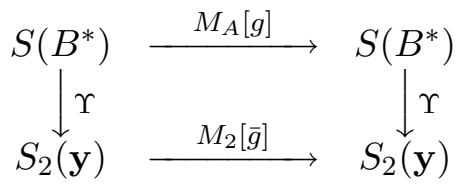

commutes for $g \in K_{B}$ and $\bar{g}$ denotes the image of $g$ in $K_{B} / K_{B}^{\prime}$. Therefore, from Subsection 4.2 , we know that $\left(m(\bar{g}) q^{j(\bar{g}) / 2} M_{A}[g], S\left(B^{*}\right)\right)$ is the representation of $K_{B}$ lifted from the Weil representation of the finite symplectic group $K_{B} / K_{B}^{\prime}$.

Now we can have a more precise realization of the generalized lattice model. Let $\mathcal{S}(B)$ be the space of locally constant, compactly supported maps $f$ from $\mathcal{W}$ to the space of complex valued functions on $B^{*}$ such that

(i) $f(w)(a+b)=\psi\left(\frac{1}{2}\langle\langle b, a\rangle) f(w)(b)\right.$ for $w \in \mathcal{W}, b \in B^{*}, a \in A$, and

(ii) $f(w)(b)=\psi\left(\frac{1}{2}\langle\langle b, w\rangle) f(b+w)(0)\right.$ for $b, b^{\prime} \in B^{*}, w \in \mathcal{W}$.

For $f \in \mathcal{S}(B)$, define $\Omega . f: \mathcal{W} \rightarrow \mathbf{C}$ by

$$
(\Omega . f)(w):=f(w)(0) .
$$

By (ii), we have $(\Omega . f)(a+w)=f(a+w)(0)=\psi\left(\frac{1}{2}\langle\langle w, a\rangle) f(w)(a)\right.$ for $a \in A \subseteq B^{*}$ and $w \in \mathcal{W}$. Then by (i), we have

$$
\begin{aligned}
\psi\left(\frac{1}{2}\langle\langle w, a\rangle) f(w)(a)\right. & =\psi\left(\frac{1}{2}\langle\langle w, a\rangle) \psi\left(\frac{1}{2}\langle\langle a, 0\rangle\rangle\right) f(w)(0)\right. \\
& \left.=\psi\left(\frac{1}{2}\langle w, a\rangle\right\rangle\right)(\Omega . f)(w) .
\end{aligned}
$$

Therefore, $\Omega$ is a map from $\mathcal{S}(B)$ to $\mathcal{S}(A)$. 
Lemma. The map $\Omega$ is an isomorphism of vector spaces and the following diagram

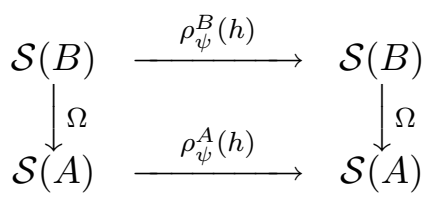

commutes for any $h \in \mathrm{H}(\mathcal{W})$.

Proof. We only need to check for $h=w \in \mathcal{W}$. Let $f \in \mathcal{S}(B)$ and $w^{\prime}$ be an element in $\mathcal{W}$. Then

$$
\begin{aligned}
\left(\rho_{\psi}^{A}(w)(\Omega . f)\right)\left(w^{\prime}\right) & =\psi\left(\frac{1}{2}\left\langle w^{\prime}, w\right\rangle\right)(\Omega . f)\left(w^{\prime}+w\right) \\
& =\psi\left(\frac{1}{2}\left\langle w^{\prime}, w\right\rangle\right) f\left(w^{\prime}+w\right)(0) \\
& =\left(\rho_{\psi}^{B}(w) . f\right)\left(w^{\prime}\right)(0) \\
& =\left(\Omega\left(\rho_{\psi}^{B}(w) . f\right)\right)\left(w^{\prime}\right) .
\end{aligned}
$$

Hence, the diagram commutes and $\Omega$ is an isomorphism.

The conclusion of this subsection is that the following diagram

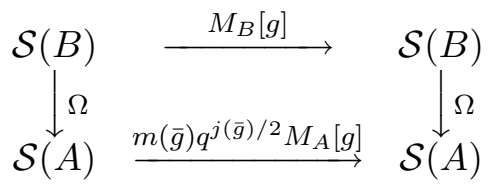

commutes for any $g \in K_{B}$.

4.4. The main identity. To prove Proposition 3.3, we need to compute $\beta_{\mathcal{V}^{\prime}}^{Y}(g)$ and $\alpha_{\mathcal{V}^{\prime}}(g)$ for $g$ in a set of representatives of $\left[U(\mathcal{V})_{L}, U(\mathcal{V})_{L}\right]$-cosets in $S U(\mathcal{V})_{L}$. The computation for $\beta_{\mathcal{V}^{\prime}}^{Y}(g)$ will be straight forward from the formula in $[\mathbf{K l}]$ Theorem 3.1. Now we describe the method for computation for $\alpha \mathcal{V}^{\prime}(g)$. Realize the generalized lattice model $\left(M_{B}, \mathcal{S}(B)\right)$ as described in Subsection 4.3. We shall properly choose a self-dual lattice $A$ in $\mathcal{W}$ (or $\mathcal{W}+\overline{\mathcal{W}})$ such that $B \subseteq A \subseteq B^{*}$. Let $\Omega: \mathcal{S}(B) \rightarrow \mathcal{S}(A)$ be the isomorphism defined in Subsection 4.3. Then we have

$$
\Omega \circ M_{B}\left[\iota_{\mathcal{V}^{\prime}}(g)\right]=m\left(\overline{\left(\mathcal{V}^{\prime}(g)\right.}\right) q^{j\left(\overline{\iota_{\mathcal{V}^{\prime}}(g)}\right) / 2} M_{A}\left[\iota \mathcal{V}^{\prime}(g)\right] \circ \Omega
$$

where $g \in U(\mathcal{V})_{L}$ and $\overline{\iota \mathcal{V}^{\prime}(g)}$ is the image of $\iota_{\mathcal{V}^{\prime}}(g)$ in $K_{B} / K_{B}^{\prime}$. Let $\Xi: \mathcal{S}(A) \rightarrow$ $\mathcal{S}(Y)$ be the isomorphism defined in Subsection 4.1. Since $\alpha_{\mathcal{V}^{\prime}}(g)$ is independent of the choice of $\Psi$, we may let $\Psi:=\Xi \circ \Omega: \mathcal{S}(B) \rightarrow \mathcal{S}(Y)$. Therefore, by substituting (4.4.a) into (3.3.a), we get

$$
M_{Y}\left[\iota \mathcal{V}^{\prime}(g)\right] \circ \Xi=\alpha_{\mathcal{V}^{\prime}}(g) m\left(\overline{\left(\mathcal{V}^{\prime}(g)\right.}\right) q^{j\left(\overline{\iota_{\mathcal{V}^{\prime}}(g)}\right) / 2} \Xi \circ M_{A}\left[\iota \mathcal{V}^{\prime}(g)\right]
$$

for $g \in U(\mathcal{V})_{L}$. The identity (4.4.b) can be applied on any function in $\mathcal{S}(A)$. In particular, we shall choose the characteristic function $f_{A} \in \mathcal{S}(A)$ and 
find the value of the identity at 0 . That is, we shall compute $\alpha_{\mathcal{V}^{\prime}}(g)$ by the following identity

$$
\left(M_{Y}\left[\iota \mathcal{V}^{\prime}(g)\right] \circ \Xi \cdot f_{A}\right)(0)=\alpha_{\mathcal{V}^{\prime}}(g) m\left(\overline{\iota \mathcal{V}^{\prime}(g)}\right) q^{j\left(\overline{\iota_{\mathcal{V}^{\prime}}(g)}\right) / 2}\left(\Xi \circ M_{A}\left[\iota \mathcal{V}^{\prime}(g)\right] . f_{A}\right)(0) .
$$

\section{Several lemmas for unitary groups.}

In this section, we introduce a few lemmas which will be used in the computation for unitary groups in Sections 6 and 7 .

5.1. . The following lemma is somewhat well-known. Recall that $\psi$ is a character of $F$ with conductoral exponent $\operatorname{ord}(\psi)=\lambda_{F}$ and $\psi_{0}$ is the character of $F$ defined by $\psi_{0}(t):=\psi(t / 2)$. It is obvious that $\operatorname{ord}(\psi)=\operatorname{ord}\left(\psi_{0}\right)$.

Lemma. Let a be an element in $\mathcal{O}_{F}^{\times}$. Then

$$
\sum_{t \in \mathcal{O}_{F} / \mathfrak{p}_{F}^{\text {ord }(a)}} \psi\left(a^{-1} \varpi_{F}^{\lambda_{F}} t^{2}\right)=q^{\operatorname{ord}(a) / 2} \gamma_{F}\left(2 a^{-1} \varpi_{F}^{\lambda_{F}}, \psi_{0}\right) \gamma_{F}\left(\psi_{0}\right) .
$$

Proof. Let $\psi^{\prime}$ be the character of $F$ defined by $\psi^{\prime}(t):=\psi\left(a^{-1} \varpi_{F}^{\lambda_{F}} t\right)=$ $\psi_{0}\left(2 a^{-1} \varpi_{F}^{\lambda_{F}} t\right)$ for $t \in F$. Then it is clear that $\operatorname{ord}\left(\psi^{\prime}\right)=\operatorname{ord}(a)$. It is well-known that

$$
\sum_{t \in \mathcal{O}_{F} / \mathfrak{p}_{F}^{\operatorname{ord}(a)}} \psi^{\prime}\left(t^{2}\right)= \begin{cases}q^{\operatorname{ord}(a) / 2}, & \text { if } \operatorname{ord}(a) \text { is even; } \\ q^{\operatorname{ord}(a) / 2} \gamma_{\mathbf{f}_{F}}\left(\bar{\psi}^{\prime}\right), & \text { if } \operatorname{ord}(a) \text { is odd. }\end{cases}
$$

This can be found, for example, in [Mn] p. 366 and p. 372. From (3) in Subsection 1.5, we conclude that

$$
\sum_{t \in \mathcal{O}_{F} / \mathfrak{p}_{F}^{\text {ord }(a)}} \psi^{\prime}\left(t^{2}\right)=q^{\operatorname{ord}(a) / 2} \gamma_{F}\left(\psi^{\prime}\right)=q^{\operatorname{ord}(a) / 2} \gamma_{F}\left(2 a^{-1} \varpi_{F}^{\lambda_{F}}, \psi_{0}\right) \gamma_{F}\left(\psi_{0}\right)
$$

5.2. An embedding. The setting of the remaining of Section 5 will be as follows. Let $\left(U(\mathcal{V}), U\left(\mathcal{V}^{\prime}\right)\right)$ be the reductive dual pairs of unitary groups such that both $\mathcal{V}, \mathcal{V}^{\prime}$ are one-dimensional over $E:=F(\delta)$ where $\Delta:=\delta^{2}$ is a nonsquare element in $F^{\times}$. We assume that $\operatorname{ord}(\Delta)=0$ or 1 . Let $\mathbf{h}, \mathbf{h}^{\prime}$ denote the forms on $\mathcal{V}, \mathcal{V}^{\prime}$ respectively.

Now we want to embed $U(\mathcal{V})$ into an isotropic unitary group in two variables. The material in this subsection is from $[\mathbf{K l}]$ Section 4 although our choice of the basis is slightly different. Let $\overline{\mathcal{V}}$ be the space $\mathcal{V}$ with the form $\overline{\mathbf{h}}:=-\mathbf{h}$. Let $v$ be a nonzero element in $\mathcal{V}$ and $\widehat{v}$ be the element in $\overline{\mathcal{V}}$ corresponding to $v$. Let $v^{\prime}$ be a nonzero element in $\mathcal{V}^{\prime}$. Let $r:=\mathbf{h}(v, v)$ and $r^{\prime}:=\mathbf{h}^{\prime}\left(v^{\prime}, v^{\prime}\right)$. We know that $r r^{\prime} \in \delta F^{\times}$. Let $\varrho$ be the element in 
$F$ such that $\delta \varrho:=r r^{\prime}$. We will choose $v, v^{\prime}$ such that $\operatorname{ord}(\varrho)=0$ or 1 . Define $\mathcal{W}:=\mathcal{V} \otimes \mathcal{V}^{\prime}$ and $\overline{\mathcal{W}}:=\overline{\mathcal{V}} \otimes \mathcal{V}^{\prime}$. Let $w_{1}:=-(2 \Delta \varrho)^{-1}(v-\widehat{v}) \otimes v^{\prime}$, $w_{2}:=(2 \Delta \varrho)^{-1} \delta(v-\widehat{v}) \otimes v^{\prime}, w_{3}:=\delta(v+\widehat{v}) \otimes v^{\prime}, w_{4}:=(v+\widehat{v}) \otimes v^{\prime}$. Let $\langle\langle\rangle$, denote the skew-symmetric form on $\mathcal{W}+\overline{\mathcal{W}}$. It is easy to check that

$$
\left\langle\left\langle w_{i}, w_{j}\right\rangle\right\rangle=\left\{\begin{aligned}
1, & \text { if }(i, j)=(1,3) \text { or }(2,4) ; \\
-1, & \text { if }(i, j)=(3,1) \text { or }(4,2) ; \\
0, & \text { otherwise. }
\end{aligned}\right.
$$

The set $\left\{w_{1}, w_{2}, w_{3}, w_{4}\right\}$ is our choice of a basis of $\mathcal{W}+\overline{\mathcal{W}}$. Let $X$ (resp. $Y$ ) be the $F$-space spanned by $\left\{w_{1}, w_{2}\right\}$ (resp. $\left\{w_{3}, w_{4}\right\}$ ). Then $\mathcal{W}+\overline{\mathcal{W}}=X+Y$ is a complete polarization.

Let $g$ be an element in $U(\mathcal{V})$. Then $g$ can be written as $x+\delta y$ for some $x, y \in F$ such that $x^{2}-\Delta y^{2}=1$. Under our assumption of the element $\Delta$, we know $x, y \in \mathcal{O}_{F}$. Then we have

$$
\iota^{\prime}(g)=\frac{1}{2}\left[\begin{array}{cccc}
x+1 & -\Delta y & -2 \Delta^{2} \varrho y & -2 \Delta \varrho(x-1) \\
-y & x+1 & 2 \Delta \varrho(x-1) & 2 \Delta \varrho y \\
-\frac{y}{2 \Delta \varrho} & \frac{x-1}{2 \Delta \varrho} & x+1 & y \\
-\frac{x-1}{2 \Delta \varrho} & \frac{y}{2 \varrho} & \Delta y & x+1
\end{array}\right] \in \operatorname{Sp}(\mathcal{W}+\overline{\mathcal{W}})
$$

with respect to the basis given above. Write $\iota_{\mathcal{V}^{\prime}}(g)=\left[\begin{array}{ll}a & b \\ c & d\end{array}\right]$ according to the complete polarization $\mathcal{W}+\overline{\mathcal{W}}=X+Y$. Then

$$
c=\frac{1}{4 \Delta \varrho}\left[\begin{array}{cc}
-y & x-1 \\
-(x-1) & \Delta y
\end{array}\right], \quad d=\frac{1}{2}\left[\begin{array}{cc}
x+1 & y \\
\Delta y & x+1
\end{array}\right] .
$$

We know that $c^{*}$ is just the transpose of $c$ under the basis. Therefore

$$
-c^{*}=\frac{1}{4 \Delta \varrho}\left[\begin{array}{cc}
y & x-1 \\
-(x-1) & -\Delta y
\end{array}\right], \quad-d \cdot c^{*}=\frac{1}{4}\left[\begin{array}{cc}
\frac{y}{\Delta \varrho} & 0 \\
0 & -\frac{y}{\varrho}
\end{array}\right] .
$$

Note that $\operatorname{det}(c)=\frac{1}{8} \Delta^{-2} \varrho^{-2}(1-x)$ and $\operatorname{det}(d)=\frac{1+x}{2}$. We know that $g=1$ if and only if $x=1$. Therefore, $\operatorname{Ker}(c)=\{0\}$ if $g \neq 1$ and $c=0$ if $g=1$. Hence, we conclude that

$$
j\left(\iota^{\prime}(g)\right)= \begin{cases}0, & \text { if } g=1 \\ 2, & \text { if } g \neq 1\end{cases}
$$

5.3. . Let $L$ (resp. $L^{\prime}$ ) be the unique good lattice in $\mathcal{V}$ (resp. $\mathcal{V}^{\prime}$ ). Let $\bar{L}$ be the corresponding good lattice in $\overline{\mathcal{V}}$. Then $L+\bar{L}$ is a good lattice in $\mathcal{V}+\overline{\mathcal{V}}$. Define

$$
B:=B\left(L+\bar{L}, L^{\prime}\right)
$$

as in Subsection 3.2. Then $B$ is a good lattice in $\mathcal{W}+\overline{\mathcal{W}}$. 
Lemma. The decomposition $X+Y$ of the space $\mathcal{W}+\overline{\mathcal{W}}$ is $B$-admissible.

Proof. Let $\lambda$ be the number given in Subsection 3.1 and $v, \widehat{v}, v^{\prime}, w_{1}, \ldots, w_{4}$ be the vectors defined in Subsection 5.2. Then it is straight forward to check that

$$
B= \begin{cases}\mathfrak{p}_{E}^{\lceil\lambda / 2\rceil} v \otimes v^{\prime}+\mathfrak{p}_{E}^{\lceil\lambda / 2\rceil} \widehat{v} \otimes v^{\prime}, & \text { if } E \text { is unramified and } \operatorname{ord}(\varrho)=0 ; \\ \mathfrak{p}_{E}^{\lfloor\lambda / 2\rfloor} v \otimes v^{\prime}+\mathfrak{p}_{E}^{\lfloor\lambda / 2\rfloor} \widehat{v} \otimes v^{\prime}, & \text { otherwise. }\end{cases}
$$

We know that $\mathfrak{p}_{E}^{k}=\mathfrak{p}_{F}^{k}+\delta \mathfrak{p}_{F}^{k}$ if $E$ is unramified and $\mathfrak{p}_{E}^{k}=\mathfrak{p}_{F}^{\lceil k / 2\rceil}+\delta \mathfrak{p}_{F}^{\lfloor k / 2\rfloor}$ if $E$ is ramified. Therefore we have the following. If $E$ is unramified and $\operatorname{ord}(\varrho)=0$, then $\lambda=\lambda_{F}$ and

$$
B=\mathfrak{p}_{F}^{\left\lceil\lambda_{F} / 2\right\rceil} v \otimes v^{\prime}+\mathfrak{p}_{F}^{\left\lceil\lambda_{F} / 2\right\rceil} \delta v \otimes v^{\prime}+\mathfrak{p}_{F}^{\left\lceil\lambda_{F} / 2\right\rceil} \widehat{v} \otimes v^{\prime}+\mathfrak{p}_{F}^{\left\lceil\lambda_{F} / 2\right\rceil} \delta \widehat{v} \otimes v^{\prime} .
$$

If $E$ is unramified and $\operatorname{ord}(\varrho)=1$, then $\lambda=\lambda_{F}$ and

$$
B=\mathfrak{p}_{F}^{\left\lfloor\lambda_{F} / 2\right\rfloor} v \otimes v^{\prime}+\mathfrak{p}_{F}^{\left\lfloor\lambda_{F} / 2\right\rfloor} \delta v \otimes v^{\prime}+\mathfrak{p}_{F}^{\left\lfloor\lambda_{F} / 2\right\rfloor} \widehat{v} \otimes v^{\prime}+\mathfrak{p}_{F}^{\left\lfloor\lambda_{F} / 2\right\rfloor} \delta \widehat{v} \otimes v^{\prime} .
$$

If $E$ is ramified and $\lambda_{F}$ is odd, then $\lambda=2 \lambda_{F}-1$ and

$$
\begin{aligned}
B= & \mathfrak{p}_{F}^{\left\lceil\left(\lambda_{F}-1\right) / 2\right\rceil} v \otimes v^{\prime}+\mathfrak{p}_{F}^{\left\lfloor\left(\lambda_{F}-1\right) / 2\right\rfloor} \delta v \otimes v^{\prime}+\mathfrak{p}_{F}^{\left\lceil\left(\lambda_{F}-1\right) / 2\right\rceil} \widehat{v} \otimes v^{\prime} \\
& +\mathfrak{p}_{F}^{\left\lfloor\left(\lambda_{F}-1\right) / 2\right\rfloor} \delta \widehat{v} \otimes v^{\prime} \\
= & \mathfrak{p}_{F}^{\left(\lambda_{F}-1\right) / 2} v \otimes v^{\prime}+\mathfrak{p}_{F}^{\left(\lambda_{F}-1\right) / 2} \delta v \otimes v^{\prime}+\mathfrak{p}_{F}^{\left(\lambda_{F}-1\right) / 2} \widehat{v} \otimes v^{\prime} \\
& +\mathfrak{p}_{F}^{\left(\lambda_{F}-1\right) / 2} \delta \widehat{v} \otimes v^{\prime} \\
= & \mathfrak{p}_{F}^{\left(\lambda_{F}+1\right) / 2} w_{1}+\mathfrak{p}_{F}^{\left(\lambda_{F}+1\right) / 2} w_{2}+\mathfrak{p}_{F}^{\left(\lambda_{F}-1\right) / 2} w_{3}+\mathfrak{p}_{F}^{\left(\lambda_{F}-1\right) / 2} w_{4} .
\end{aligned}
$$

If $E$ is ramified and $\lambda_{F}$ is even, then $\lambda=2 \lambda_{F}-1$ and

$$
\begin{aligned}
B= & \mathfrak{p}_{F}^{\left\lceil\left(\lambda_{F}-1\right) / 2\right\rceil} v \otimes v^{\prime}+\mathfrak{p}_{F}^{\left\lfloor\left(\lambda_{F}-1\right) / 2\right\rfloor} \delta v \otimes v^{\prime}+\mathfrak{p}_{F}^{\left\lceil\left(\lambda_{F}-1\right) / 2\right\rceil} \widehat{v} \otimes v^{\prime} \\
& +\mathfrak{p}_{F}^{\left\lfloor\left(\lambda_{F}-1\right) / 2\right\rfloor} \delta \widehat{v} \otimes v^{\prime} \\
= & \mathfrak{p}_{F}^{\lambda_{F} / 2} v \otimes v^{\prime}+\mathfrak{p}_{F}^{\lambda_{F} / 2-1} \delta v \otimes v^{\prime}+\mathfrak{p}_{F}^{\lambda_{F} / 2} \widehat{v} \otimes v^{\prime}+\mathfrak{p}_{F}^{\lambda_{F} / 2-1} \delta \widehat{v} \otimes v^{\prime} \\
= & \mathfrak{p}_{F}^{\lambda_{F} / 2+1} w_{1}+\mathfrak{p}_{F}^{\lambda_{F} / 2} w_{2}+\mathfrak{p}_{F}^{\lambda_{F} / 2-1} w_{3}+\mathfrak{p}_{F}^{\lambda_{F} / 2} w_{4} .
\end{aligned}
$$

So it is clear that the basis $\left\{w_{1}, w_{2}, w_{3}, w_{4}\right\}$ is $B$-admissible. $X=F w_{1}+F w_{2}$ and $Y=F w_{3}+F w_{4}$. Hence the decomposition $X+Y$ is $B$-admissible.

5.4. . Define $L_{Y}:=B \cap Y$, which is a lattice in $Y$. Let $L_{X}$ be the dual lattice of $L_{Y}$ in $X$. Therefore, the lattice

$$
A:=L_{X}+L_{Y}
$$

is a self-dual lattice in $\mathcal{W}+\overline{\mathcal{W}}$. It is clear that $B \subseteq A \subseteq B^{*}$ and $B \cap Y=$ $A \cap Y=L_{Y}$. 
Lemma. Let $g$ be an element in $U(\mathcal{V})$. Then

$$
\left(\iota \mathcal{V}^{\prime}(g) \cdot A+A\right) \cap X=L_{X} .
$$

Proof. Let $g^{\prime}:=\iota \mathcal{V}^{\prime}(g) \in \operatorname{Sp}(\mathcal{W}+\overline{\mathcal{W}})$. Since now $\mathcal{V}$ is anisotropic, we have $\iota \mathcal{V}^{\prime}(U(\mathcal{V}))=\iota_{\mathcal{V}^{\prime}}\left(U(\mathcal{V})_{L}\right) \subseteq K_{B}$. So $g^{\prime}$ stabilizes the lattice $B$. Therefore, $B \subseteq g^{\prime} . A \cap A \subseteq A$. But we know that $B \cap Y=A \cap Y=L_{Y}$. Hence, $\left(g^{\prime} \cdot A \cap A\right) \cap Y=L_{Y}$. Note that $g^{\prime} \cdot A+A=\left(g^{\prime} \cdot A\right)^{*}+A^{*}=\left(g^{\prime} \cdot A \cap A\right)^{*}$. Therefore $\left(g^{\prime} \cdot A+A\right) \cap X=L_{X}$.

\section{5. .}

Lemma. Let $g$ be an element in $U(\mathcal{V})$. Then

$$
\left(\Xi \circ M_{A}\left[\iota^{\prime}(g)\right] \cdot f_{A}\right)(0)=\mu_{X}\left(L_{X}\right) \mu\left(\iota \mathcal{V}^{\prime}(g) \cdot A \cap A\right)
$$

where $\Xi$ is the map defined in Subsection 4.1 and $f_{A} \in \mathcal{S}(A)$ is the characteristic function on $A$.

Proof. Let $g^{\prime}:=\iota \mathcal{V}^{\prime}(g) \in \operatorname{Sp}(\mathcal{W})$ and $I_{0}:=\left(\Xi \circ M_{A}\left[g^{\prime}\right] . f_{A}\right)(0)$. Then

$$
I_{0}=\int_{X}\left(M_{A}\left[g^{\prime}\right] \cdot f_{A}\right)(x) d \mu_{X}(x)
$$

from (4.1.a). It is clear that the support of the function $M_{A}\left[g^{\prime}\right] . f_{A}$ is contained in $g^{\prime} \cdot A+A$. By Lemma 5.4, we know that $\left(g^{\prime} \cdot A+A\right) \cap X=L_{X}$. Therefore

$$
\begin{aligned}
I_{0} & =\int_{L_{X}}\left(M_{A}\left[g^{\prime}\right] \cdot f_{A}\right)(x) d \mu_{X}(x) \\
& =\int_{L_{X}} \int_{A} \psi\left(\frac{1}{2}\langle\langle a, x\rangle) f_{A}\left(g^{\prime-1} \cdot(a+x)\right) d \mu(a) d \mu_{X}(x) .\right.
\end{aligned}
$$

If $x \in L_{X}$, then $a^{\prime}:=a+x$ is in $A$. Therefore

$$
\begin{aligned}
I_{0} & =\int_{L_{X}} \int_{A} \psi\left(\frac{1}{2}\left\langle\left\langle a^{\prime}-x, x\right\rangle\right) f_{A}\left(g^{\prime-1} . a^{\prime}\right) d \mu\left(a^{\prime}\right) d \mu_{X}(x)\right. \\
& =\int_{L_{X}} \int_{g^{\prime} \cdot A \cap A} d \mu\left(a^{\prime}\right) d \mu_{X}(x)=\mu_{X}\left(L_{X}\right) \mu\left(g^{\prime} \cdot A \cap A\right) .
\end{aligned}
$$

\section{6. .}

Lemma. Let $\Xi$ be the map defined in (4.1.a) and $f_{A} \in \mathcal{S}(A)$ (resp. $f_{Y} \in$ $\mathcal{S}(Y))$ be the characteristic function of $A($ resp. $Y)$. Then $\Xi . f_{A}=\mu_{X}\left(L_{X}\right) f_{Y}$.

Proof. For $y \in Y$, by the definition we have

$$
\left(\Xi . f_{A}\right)(y)=\int_{X} \psi\left(\frac{1}{2}\langle\langle x, y\rangle) f_{A}(x+y) d \mu_{X}(x) .\right.
$$


From the definition, $f_{A}(x+y)$ is not zero if and only if $x+y \in A$. Now $x \in X$, $y \in Y$, so $x+y \in A$ if and only if $x \in L_{X}, y \in L_{Y}$. Therefore if $x+y \in A$, we have $\psi\left(\frac{1}{2}\langle\langle x, y\rangle\rangle\right) f_{A}(x+y)=1$. Hence we have $\left(\Xi . f_{A}\right)(y)=\mu_{X}\left(L_{X}\right)$ if $y \in L_{Y}$ and $\left(\Xi . f_{A}\right)(y)=0$ if $y \notin L_{Y}$.

5.7. The normalized Haar measure. The action $M_{Y}[g]$ defined in (2.2.b) depends on normalization of the Haar measure $\mu_{g}$ on the space $X / \operatorname{Ker}(c)$. The measure $\mu_{g}$ is given explicitly in $[\mathbf{R R}]$ Theorem 3.5. We describe the normalization in our situation. Let $g$ be an element in $U(\mathcal{V})$. Write $\iota \mathcal{V}^{\prime}(g)=$ $\left[\begin{array}{ll}a & b \\ c & d\end{array}\right] \in \operatorname{Sp}(\mathcal{W}+\overline{\mathcal{W}})$ with respect to the decomposition $X+Y$ of $\mathcal{W}+\overline{\mathcal{W}}$.

From the computation in Subsection 5.4, we know that $L_{X}=\mathfrak{p}_{F}^{\lambda_{1}} w_{1}+\mathfrak{p}_{F}^{\lambda_{2}} w_{2}$ and $L_{Y}=\mathfrak{p}_{F}^{\lambda_{F}-\lambda_{1}} w_{3}+\mathfrak{p}_{F}^{\lambda_{F}-\lambda_{2}} w_{4}$ where

$$
\begin{aligned}
& \lambda_{2}:= \begin{cases}\left\lfloor\lambda_{F} / 2\right\rfloor, & \text { if } E \text { is unramified, } \operatorname{ord}(\varrho)=0 \text { and } \lambda_{F} \text { is odd } \\
\left\lceil\lambda_{F} / 2\right\rceil, & \text { otherwise, }\end{cases} \\
& \lambda_{1}:= \begin{cases}\lambda_{2}+1, & \text { if } E \text { is ramified and } \lambda_{F} \text { is even; } \\
\lambda_{2}, & \text { otherwise. }\end{cases}
\end{aligned}
$$

Following $[\mathbf{R R}]$, we normalize a Haar measure $\mu_{X}$ on the additive group $X$ by

$$
\mu_{X}\left(L_{X}\right)=q^{-\lambda_{0}}
$$

where $\lambda_{0}:=\lambda_{1}+\lambda_{2}-\lambda_{F}$. Let $\mu_{\{0\}}$ denote the measure of $\{0\}$ such that $\mu_{\{0\}}(\{0\})=1$. Then from $[\mathbf{R R}]$, we have

$$
\mu_{\iota_{\mathcal{V}^{\prime}}(g)}= \begin{cases}|\operatorname{det}(a)|^{1 / 2} \mu_{\{0\}}, & \text { if } c=0 ; \\ |\operatorname{det}(c)|^{1 / 2} \mu_{X}, & \text { if } \operatorname{Ker}(c)=\{0\}\end{cases}
$$

where $|\cdot|$ denotes the normalized multiplicative valuation of $F$, i.e., $|0|=0$ and $|t|=q^{-\operatorname{ord}(t)}$ for $t \in F^{\times}$. Formula (5.7.a) looks different from the formula in $[\mathbf{R R}]$. This is because we use the left action of $\operatorname{Sp}(\mathcal{W}+\overline{\mathcal{W}})$ on $\mathcal{W}+\overline{\mathcal{W}}$ instead of right action used in $[\mathbf{R R}]$.

5.8. . For $g:=x+\delta y \in U(\mathcal{V})$ and $g \neq 1$, define

$$
L_{X}[g]:=\left\{x \in X \mid-c^{*} . x \in L_{Y}\right\}
$$

where $-c^{*}$ is as given in (5.2.b). It is clear that $L_{X}[g]$ is a lattice in $X$ since $-c^{*}$ is an isomorphism of vector spaces.

Lemma. Suppose that $g \neq 1$. If $x \not \equiv 1 \bmod \mathfrak{p}_{F}$, then $L_{X}[g] \subseteq L_{X}$. If $x \equiv 1 \bmod \mathfrak{p}_{F}$, then $L_{X} \subseteq L_{X}[g]$. 
Proof. First, we suppose that $g=-1$. Hence, $y=0$. From (5.2.a), we see that $L_{Y} \subseteq B^{*} \cap Y=\iota_{\mathcal{V}^{\prime}}(g) \cdot\left(B^{*} \cap X\right)=-c^{*} . L_{X}$. So $L_{X}[g] \subseteq L_{X}$ in this case.

For the remaining of the proof, we assume that $y \neq 0$. Hence we have $\operatorname{ord}(y)=0$ if $x \not \equiv 1 \bmod \mathfrak{p}_{F}$, and $\operatorname{ord}(1-x)=2 \operatorname{ord}(y)+\operatorname{ord}(\Delta)$ if $x \equiv 1 \bmod \mathfrak{p}_{F}$ because $x^{2}-\Delta y^{2}=1$. From (5.2.b) and the result in Subsection 5.7, it is clear that

$$
L_{X}[g]= \begin{cases}\mathfrak{p}_{F}^{\lambda_{F}-\lambda_{1}+\operatorname{ord}(\Delta \varrho / y)} w_{1}+\mathfrak{p}_{F}^{\lambda_{F}-\lambda_{2}+\operatorname{ord}(\varrho / y)} w_{2}, & \text { if } x \equiv 1 \bmod \mathfrak{p}_{F} \\ \mathfrak{p}_{F}^{\lambda_{F}-\lambda_{1}+\operatorname{ord}(\Delta \varrho)} w_{1}+\mathfrak{p}_{F}^{\lambda_{F}-\lambda_{2}+\operatorname{ord}(\Delta \varrho)} w_{2}, & \text { if } x \neq 1 \bmod \mathfrak{p}_{F}\end{cases}
$$

(1) Suppose that $E$ is unramified, $\operatorname{ord}(\varrho)=0$ and $\lambda_{F}$ is odd. Then $\lambda_{1}=$ $\lambda_{2}=\frac{\lambda_{F}-1}{2}$. Since now $\operatorname{ord}(y)>0$ if $x \equiv 1 \bmod \mathfrak{p}_{F}$ and $\operatorname{ord}(y)=0$ if $x \not \equiv 1 \bmod \mathfrak{p}_{F}$. The lemma is clear in this case.

(2) Suppose that $E$ is ramified and $\lambda_{F}$ is even. Then $\lambda_{1}=\frac{\lambda_{F}}{2}+1$ and $\lambda_{2}=\frac{\lambda_{F}}{2}$. We also have ord $(\varrho)=0$ under our assumption. Again, the lemma is obvious in this case.

(3) Suppose we are in the situation other than the previous two cases. Then $\lambda_{1}=\lambda_{2}=\left\lceil\frac{\lambda_{F}}{2}\right\rceil$. It is straight to check that the lemma is true in this case.

\section{9. .}

Lemma. Suppose that $g:=x+y \delta$ is an element of $U(\mathcal{V})$ for some $x, y \in F$ and $g \neq 1$. Then

$$
\begin{aligned}
& \left(M_{Y}\left[\iota \mathcal{V}^{\prime}(g)\right] \cdot f_{Y}\right)(0) \\
& = \begin{cases}q^{-j\left(\overline{\iota_{\mathcal{V}^{\prime}}(g)}\right) / 2}, & \text { if } x \neq 1 \bmod \mathfrak{p}_{F} ; \\
\gamma_{F}\left(-\Delta, \psi_{0}\right)(\Delta, 2 y \varrho)_{F}\left(\frac{-1}{\mathbf{f}_{F}}\right)^{\overline{\operatorname{ord}}\left(\psi_{0}\right)}, & \text { if } x \equiv 1 \bmod \mathfrak{p}_{F} .\end{cases}
\end{aligned}
$$

Proof. Let $g^{\prime}:=\iota_{\mathcal{V}^{\prime}}(g)=\left[\begin{array}{ll}a & b \\ c & d\end{array}\right] \in \operatorname{Sp}(\mathcal{W}+\overline{\mathcal{W}})$. From (2.2.b), we have

$$
\begin{aligned}
\left(M_{Y}\left[g^{\prime}\right] . f_{Y}\right)(0) & =\int_{X / \operatorname{Ker}(c)} \psi\left(\frac{1}{2}\left\langle\left\langle-c^{*} \cdot x, d^{*} \cdot x\right\rangle\right) f_{Y}\left(-c^{*} \cdot x\right) d \mu_{g^{\prime}}(x)\right. \\
& =\int_{X / \operatorname{Ker}(c)} \psi\left(\frac{1}{2}\left\langle\left\langle-d \cdot c^{*} \cdot x, x\right\rangle\right) f_{Y}\left(-c^{*} \cdot x\right) d \mu_{g^{\prime}}(x) .\right.
\end{aligned}
$$

From our assumption that $g \neq 1$, we know that $\operatorname{Ker}(c)=\{0\}$. Then

$$
\left(M_{Y}\left[g^{\prime}\right] \cdot f_{Y}\right)(0)=\int_{L_{X}[g]} \psi\left(\frac{1}{2}\left\langle-d \cdot c^{*} . x, x\right\rangle\right) d \mu_{g^{\prime}}(x)
$$

where $L_{X}[g]$ is defined in (5.8.a). It is clear that $-d . c^{*} . L_{X}[g] \subseteq L_{Y}$. 
Suppose that $x \not \equiv 1 \bmod \mathfrak{p}_{F}$. So $L_{X}[g] \subseteq L_{X}$ by Lemma 5.8. Then $\psi\left(\frac{1}{2}\left\langle\left\langle-d . c^{*} . x, x\right\rangle\right)=1\right.$ for $x \in L_{X}[g]$. Therefore,

$$
\left(M_{Y}\left[g^{\prime}\right] . f_{Y}\right)(0)=\int_{L_{X}[g]} d \mu_{g^{\prime}}(x)=\mu_{g^{\prime}}\left(L_{X}[g]\right)=|\operatorname{det}(c)|^{1 / 2} \mu_{X}\left(L_{X}[g]\right) .
$$

Clearly, $|\operatorname{det}(c)|^{1 / 2}=q^{\operatorname{ord}(\Delta \varrho)}$ and $\mu_{X}\left(L_{X}[g]\right)=\mu_{X}\left(L_{X}\right) q^{l}$ where $l=\lambda_{1}-\left(\lambda_{F}-\lambda_{1}+\operatorname{ord}(\Delta \varrho)\right)+\lambda_{2}-\left(\lambda_{F}-\lambda_{2}+\operatorname{ord}(\Delta \varrho)\right)=2\left(\lambda_{0}-\operatorname{ord}(\Delta \varrho)\right)$. Recall that $\mu_{X}\left(L_{X}\right)=q^{-\lambda_{0}}$. Therefore $\left(M_{Y}\left[g^{\prime}\right] . f_{Y}\right)(0)=q^{\lambda_{0}-\operatorname{ord}(\Delta \varrho)}$. It is known that $-j\left(\overline{\iota_{\mathcal{V}^{\prime}}(g)}\right)$ is equal to -2 if $\lambda_{F}+\operatorname{ord}(\varrho)$ is odd and $E$ is an unramified extension, equal to 0 otherwise. Hence it is straight forward to check that $\lambda_{0}-\operatorname{ord}(\Delta \varrho)$ is really equal to $-j\left(\overline{\left(\mathcal{V}^{\prime}(g)\right.}\right) / 2$.

Suppose that $x \equiv 1 \bmod \mathfrak{p}_{F}$. So $L_{X} \subseteq L_{X}[g]$ by Lemma 5.8. We know that $L_{X}[g]=\mathfrak{p}_{F}^{k_{1}} w_{1}+\mathfrak{p}_{F}^{k_{2}} w_{2}$ where $k_{1}=\lambda_{F}-\lambda_{1}+\operatorname{ord}(\Delta \varrho / y), k_{2}=\lambda_{F}-$ $\lambda_{2}+\operatorname{ord}(\varrho / y)$. Define $L_{X}^{\prime}[g]:=\mathfrak{p}_{F}^{l_{1}+k_{1}} w_{1}+\mathfrak{p}_{F}^{l_{2}+k_{2}} w_{2}$ where $l_{1}:=-2 k_{1}+\lambda_{F}-$ $\operatorname{ord}(y / \Delta \varrho)$ and $l_{2}:=-2 k_{2}+\lambda_{F}-\operatorname{ord}(y / \varrho)$. Then by (5.9.a) and (5.2.b), we have

$$
\begin{aligned}
\left(M_{Y}\left[g^{\prime}\right] . f_{Y}\right)(0) & =|\operatorname{det}(c)|^{1 / 2} \mu_{X}\left(L_{X}^{\prime}[g]\right) \sum_{x \in L_{X}[g] / L_{X}^{\prime}[g]} \psi\left(\frac{1}{2}\left\langle\left\langle-d \cdot c^{*} \cdot x, x\right\rangle\right)\right) \\
& =|\operatorname{det}(c)|^{1 / 2} \mu_{X}\left(L_{X}^{\prime}[g]\right) I_{1} I_{2}
\end{aligned}
$$

where

$$
\begin{aligned}
& I_{1}:=\sum_{t_{1} \in \mathfrak{p}_{F}^{k_{1} / \mathfrak{p}_{F}^{l_{1}+k_{1}}}} \psi\left(\left\langle\left\langle\frac{y}{16 \Delta \varrho} t_{1} w_{3}, t_{1} w_{1}\right\rangle\right\rangle\right)=\sum_{t_{1} \in \mathcal{O}_{F} / \mathfrak{p}_{F}^{l_{1}}} \psi\left(\frac{-y}{16 \Delta \varrho} \varpi_{F}^{2 k_{1}} t_{1}^{2}\right), \\
& I_{2}:=\sum_{t_{2} \in \mathfrak{p}_{F}^{k_{2} / \mathfrak{p}_{F}^{l_{2}+k_{2}}} \psi} \psi\left(\left\langle\left\langle\frac{-y}{16 \varrho} t_{2} w_{4}, t_{2} w_{2}\right\rangle\right\rangle\right)=\sum_{t_{2} \in \mathcal{O}_{F} / \mathfrak{p}_{F}^{l_{2}}} \psi\left(\frac{y}{16 \varrho} \varpi_{F}^{2 k_{2}} t_{2}^{2}\right) .
\end{aligned}
$$

By the results in Subsections 1.5 and 5.1, we have

$$
\begin{aligned}
& I_{1}=q^{l_{1} / 2} \gamma_{F}\left(-2 y \Delta \varrho, \psi_{0}\right) \gamma_{F}\left(\psi_{0}\right) \quad \text { and } \\
& I_{2}=q^{l_{2} / 2} \gamma_{F}\left(2 y \varrho, \psi_{0}\right) \gamma_{F}\left(\psi_{0}\right) .
\end{aligned}
$$

So

$$
\begin{aligned}
& \left(M_{Y}\left[g^{\prime}\right] . f_{Y}\right)(0) \\
& =|\operatorname{det}(c)|^{1 / 2} \mu_{X}\left(L_{X}^{\prime}[g]\right) q^{\left(l_{1}+l_{2}\right) / 2} \gamma_{F}\left(-2 y \Delta \varrho, \psi_{0}\right) \gamma_{F}\left(2 y \varrho, \psi_{0}\right) \gamma_{F}\left(\psi_{0}\right)^{2} .
\end{aligned}
$$

Clearly, $|\operatorname{det}(c)|^{1 / 2}=q^{-\operatorname{ord}\left(y^{2} / \Delta \varrho^{2}\right) / 2}$ and $\mu_{X}\left(L_{X}^{\prime}[g]\right) q^{\left(l_{1}+l_{2}\right) / 2}=\mu_{X}\left(L_{X}\right) q^{l}$ where

$$
l=\lambda_{1}+\lambda_{2}-l_{1}-l_{2}-k_{1}-k_{2}+\frac{l_{1}+l_{2}}{2}=\lambda_{1}+\lambda_{2}-\lambda_{F}+\frac{\operatorname{ord}\left(y^{2} / \Delta \varrho^{2}\right)}{2} .
$$


Recall that $\mu_{X}\left(L_{X}\right):=q^{-\lambda_{0}}$ and $\lambda_{0}:=\lambda_{1}+\lambda_{2}-\lambda_{F}$. Therefore,

$$
\left(M_{Y}\left[g^{\prime}\right] . f_{Y}\right)(0)=\gamma_{F}\left(-2 y \Delta \varrho, \psi_{0}\right) \gamma_{F}\left(2 y \varrho, \psi_{0}\right) \gamma_{F}\left(\psi_{0}\right)^{2} \text {. }
$$

We know that $\gamma_{F}\left(\psi_{0}\right)^{2}=\left(\frac{-1}{\mathbf{f}_{F}}\right)^{\overline{\operatorname{ord}}\left(\psi_{0}\right)}$ and

$$
\begin{aligned}
\gamma_{F}\left(-2 y \Delta \varrho, \psi_{0}\right) \gamma_{F}\left(2 y \varrho, \psi_{0}\right) & =\gamma_{F}\left(-\Delta, \psi_{0}\right)(-2 y \Delta \varrho, 2 y \varrho)_{F} \\
& =\gamma_{F}\left(-\Delta, \psi_{0}\right)(\Delta, 2 y \varrho)_{F}
\end{aligned}
$$

from Subsection 1.5. Hence, the lemma is proved.

\section{Proof of Proposition 3.3 for unitary groups: Part I.}

In this section, we assume that $D=E$ is an unramified quadratic extension of $F$. We assume that $E:=F(\delta)$ where $\delta^{2}:=\Delta$ is a nonsquare element in $F^{\times}$such that $\operatorname{ord}(\Delta)=0$. As usual, fix a nontrivial character $\psi$ of $F$ with conductoral exponent $\lambda_{F}$. We compute $\beta_{\mathcal{V}^{\prime}}^{Y}(g)$ and $\alpha_{\mathcal{V}^{\prime}}(g)$ for several cases in Subsection 6.1-6.6 and prove Proposition 3.3 for unitary group $U(\mathcal{V})$ with respect to an unramified extension in Subsection 6.7. As usual, let $L$ (resp. $\left.L^{\prime}\right)$ be a good lattice in $\mathcal{V}\left(\operatorname{resp} . \mathcal{V}^{\prime}\right)$.

6.1. . In this subsection, we keep the notation and the assumption in Subsection 5.2 .

Lemma. Suppose that both $\mathcal{V}, \mathcal{V}^{\prime}$ are one-dimensional, $g$ is an element in $U(\mathcal{V})$ and $g \neq 1$. Let $\beta_{\mathcal{V}^{\prime}}^{Y}$ be defined with respect to a character $\chi$ of $E^{\times}$ such that $\left.\chi\right|_{F^{\times}}=\epsilon_{E / F}$. Write $g=t=x+\delta y$ for some $t \in E^{(1)}$ and some $x, y \in F$. Let $\varrho$ be as defined in Subsection 5.2. Then

$$
\begin{aligned}
& \beta_{\mathcal{V}^{\prime}}^{Y}(g)= \begin{cases}\chi((t-1) \delta), & \text { if } \operatorname{ord}\left(\psi_{0}\right)+\operatorname{ord}(\varrho) \text { is even; } \\
-\chi((t-1) \delta), & \text { if } \operatorname{ord}\left(\psi_{0}\right)+\operatorname{ord}(\varrho) \text { is odd, }\end{cases} \\
& \alpha_{\mathcal{V}^{\prime}}(g)= \begin{cases}1, & \text { if } x \neq \equiv 1 \bmod \mathfrak{p}_{F} \text { and } \\
\left(\frac{2 x-2}{\mathbf{f}_{F}}\right), & \operatorname{ord}\left(\psi_{0}\right)+\operatorname{ord}(\varrho) \text { is even; } \\
\gamma_{F}\left(-\Delta, \psi_{0}\right)(\Delta, 2 y \varrho)_{F}\left(\frac{-1}{\mathbf{f}_{F}}\right)^{\overline{\operatorname{ord}}\left(\psi_{0}\right)}, & \text { if } x \neq 1 \bmod \mathfrak{p}_{F} \text { and }\end{cases}
\end{aligned}
$$

Proof. Let $v, r$ be as given in Subsection 5.2. Let $v_{1}:=(2 r)^{-1}(v-\widehat{v})$, $v_{2}:=v+\widehat{v}$ be two elements in $\mathcal{V}+\overline{\mathcal{V}}$. Then the set $\left\{v_{1}, v_{2}\right\}$ is a standard $E$-basis of the $\epsilon$-hermitian space $\mathcal{V}+\overline{\mathcal{V}}$ and

$$
g=\frac{1}{2}\left[\begin{array}{cc}
t+1 & 2 r(t-1) \\
(2 r)^{-1}(t-1) & t+1
\end{array}\right] \in U(\mathcal{V}+\overline{\mathcal{V}})
$$


with respect to the basis $\left\{v_{1}, v_{2}\right\}$. Therefore $j(g)=1$ because we assume that $g \neq 1$. Suppose that $\epsilon=1$. From $[\mathbf{K l}]$ Theorem 3.1 and the facts that $j(g)=1$ and $\mathcal{V}^{\prime}$ is one-dimensional, we have

$$
\begin{aligned}
\beta_{\mathcal{V}^{\prime}}^{Y}(g) & =\chi(x(g)) \chi(\delta)\left(\Delta, \operatorname{det}\left(\mathcal{V}^{\prime}\right) \delta\right)_{F}^{-1} \gamma_{F}\left(-\Delta, \psi_{0}\right)^{-1} \gamma_{F}\left(-1, \psi_{0}\right) \\
& =\chi(x(g)) \chi(\delta)\left(\Delta, r^{\prime} \delta\right)_{F} \gamma_{F}\left(\Delta, \psi_{0}\right)^{-1}(-1,-\Delta)_{F}
\end{aligned}
$$

where the second equality follows from (4) in Subsection 1.5. From the definition in $[\mathbf{K l}]$ p. 371 , we know that $x(g)=(4 r)^{-1}(t-1)$. Because $E$ is an unramified quadratic extension, we have $(-1,-\Delta)_{F}=(-1,-1)_{F}=1$, and $\gamma_{F}\left(\Delta, \psi_{0}\right)=\left(\frac{\bar{\Delta}}{\mathbf{f}_{F}}\right)^{\overline{\operatorname{ord}}\left(\psi_{0}\right)}$ by (6) in Subsection 1.5. Therefore,

$$
\beta_{\mathcal{V}^{\prime}}^{Y}(g)=\chi((t-1) \delta)\left(\Delta, r r^{\prime} \delta\right)_{F}\left(\frac{\bar{\Delta}}{\mathbf{f}_{F}}\right)^{-\overline{\operatorname{ord}}\left(\psi_{0}\right)} .
$$

Since $\Delta$ is not a square element, we have $\left(\frac{\bar{\Delta}}{\mathbf{f}_{F}}\right)=-1$. We know $r r^{\prime} \delta=\varrho \Delta$ and $(\Delta, \Delta)_{F}=1$. Therefore,

$$
\beta_{\mathcal{V}^{\prime}}^{Y}(g)=\chi((t-1) \delta)(\Delta, \varrho)_{F}(-1)^{\overline{\operatorname{ord}}\left(\psi_{0}\right)} .
$$

If $\operatorname{ord}\left(\psi_{0}\right)+\operatorname{ord}(\varrho)$ is even, then either both $\operatorname{ord}\left(\psi_{0}\right), \operatorname{ord}(\varrho)$ are even or both are odd. Therefore, $(\Delta, \varrho)_{F}(-1)^{\overline{\operatorname{ord}}\left(\psi_{0}\right)}=1$. If $\operatorname{ord}\left(\psi_{0}\right)+\operatorname{ord}(\varrho)$ is odd, then it is clear that $(\Delta, \varrho)_{F}(-1)^{\overline{\operatorname{ord}}\left(\psi_{0}\right)}=-1$. If $\epsilon=-1$, by the same computation it is not difficult to see that (6.1.b) is also true.

Next, we want to compute $\alpha_{\mathcal{V}^{\prime}}(g)$. Now $L$ is the unique good lattice in $\mathcal{V}$. Let $\bar{L}$ the good lattice in $\overline{\mathcal{V}}$ corresponding to $L$. Define $B:=B\left(L+\bar{L}, L^{\prime}\right)$. Let $A$ be the self-dual lattice in $\mathcal{W}+\overline{\mathcal{W}}$ defined in Subsection 5.4. We know that $B \subseteq A \subseteq B^{*}$. Suppose that $x \not \equiv 1 \bmod \mathfrak{p}_{F}$. It is clear that $\mu\left(\iota \mathcal{V}^{\prime}(g) . A \cap\right.$ $A)=q^{-j\left(\overline{\iota_{\mathcal{V}^{\prime}}(g)}\right)}$. Combining Lemmas 5.5, 5.6 and 5.9, we conclude that

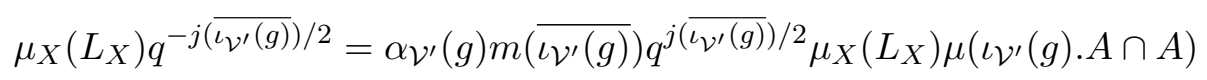

from (4.4.c). Hence,

$$
\alpha_{\mathcal{V}^{\prime}}(g) m\left(\overline{\iota_{\mathcal{V}^{\prime}}(g)}\right)=1
$$

Now we have the following two cases.

(i) First suppose that $\operatorname{ord}\left(\psi_{0}\right)+\operatorname{ord}(\varrho)$ is even. It is easy to see that $B=A$ by the computation in Subsection 5.3. Therefore $m\left(\overline{\iota \mathcal{V}^{\prime}(g)}\right)=1$ and $j\left(\overline{\iota \mathcal{V}^{\prime}}(g)\right)=0$. Hence $\alpha \mathcal{V}^{\prime}(g)=1$ by (6.1.c).

(ii) Suppose that $\operatorname{ord}\left(\psi_{0}\right)+\operatorname{ord}(\varrho)$ is odd. Then it is easy to see that $A / B$ is two-dimensional. So $B^{*} / B$ is four-dimensional. Clearly, we have $j\left(\overline{\left(\mathcal{V}^{\prime}(g)\right.}\right)=2$ from (5.2.a) because $g \neq 1$. Hence $\gamma_{\mathbf{f}_{F}}\left(\psi_{0}\right)^{-j\left(\overline{\iota_{\mathcal{L}^{\prime}}(g)}\right)}=$ $\left(\frac{-1}{\mathbf{f}_{F}}\right)^{-1}=\left(\frac{-1}{\mathbf{f}_{F}}\right)$ by (1) in Subsection 1.5. We also know that 
$\gamma_{\mathbf{f}_{F}}\left(x\left(\overline{\iota_{\mathcal{V}^{\prime}}(g)}\right), \psi_{0}\right)=\left(\frac{x\left(\overline{\iota_{\mathcal{V}^{\prime}}(g)}\right)}{\mathbf{f}_{F}}\right)$ by (2) of Subsection 1.5. Therefore,

$$
m\left(\overline{\iota \mathcal{L}^{\prime}}(g)\right)=\left(\frac{x\left(\overline{\iota_{\mathcal{V}^{\prime}}(g)}\right)}{\mathbf{f}_{F}}\right)\left(\frac{-1}{\mathbf{f}_{F}}\right)=\left(\frac{-x\left(\overline{\iota_{\mathcal{V}^{\prime}}(g)}\right)}{\mathbf{f}_{F}}\right) .
$$

Write $\iota_{\mathcal{V}^{\prime}}(g)=\left[\begin{array}{ll}a & b \\ c & d\end{array}\right]$ as in Subsection 5.2. Then $\overline{\iota_{\mathcal{V}^{\prime}}(g)}=\left[\begin{array}{ll}\bar{a} & \bar{b} \\ \bar{c} & \bar{d}\end{array}\right]$. We have $\operatorname{Ker}(\bar{c})=\{0\}$. In this case $x\left(\overline{\iota \mathcal{V}^{\prime}(g)}\right)=\operatorname{det}(\bar{c})$. From Subsection 5.2, we know $\operatorname{det}(\bar{c})=\overline{8^{-1}(\Delta \varrho)^{-2}(1-x)}$. Therefore,

$$
m\left(\overline{\iota \mathcal{V}^{\prime}(g)}\right)=\left(\frac{\overline{8^{-1}(\Delta \varrho)^{-2}(x-1)}}{\mathbf{f}_{F}}\right)=\left(\frac{\overline{2 x-2}}{\mathbf{f}_{F}}\right)=\left(\frac{\overline{2 x-2}}{\mathbf{f}_{F}}\right)^{-1} .
$$

Hence, we conclude $\alpha_{\mathcal{V}^{\prime}}(g)=\left(\frac{\overline{2 x-2}}{\mathbf{f}_{F}}\right)$ by (6.1.c).

Now we assume that $g \neq 1$ and $x \equiv 1 \bmod \mathfrak{p}_{F}$. In this case, it is clear that $\overline{\iota_{\mathcal{V}^{\prime}}(g)}=1$. So $m\left(\overline{\left(\mathcal{V}^{\prime}(g)\right.}\right)=1$ and $j\left(\overline{\iota_{\mathcal{V}^{\prime}}(g)}\right)=0$. By the computation in Lemma 5.5, 5.6 and 5.9, we conclude that

$$
\alpha_{\mathcal{V}^{\prime}}(g)=\gamma_{F}\left(-\Delta, \psi_{0}\right)(\Delta, 2 y \varrho)_{F}\left(\frac{-1}{\mathbf{f}_{F}}\right)^{\overline{\operatorname{ord}}\left(\psi_{0}\right)} .
$$

Hence the lemma is obtained.

6.2. . In this subsection, we assume that $\mathcal{V}$ is one-dimensional and $\mathcal{V}^{\prime}$ twodimensional isotropic. Let $v, \widehat{v}$ and $r$ be as given in Subsection 5.2.

Lemma. Suppose that $\mathcal{V}$ is one-dimensional and $\mathcal{V}^{\prime}$ two-dimensional isotropic. Suppose that $g:=t \in E^{(1)}$ is an element in $U(\mathcal{V})$ and $g \neq 1$. Let $\beta_{\mathcal{V}^{\prime}}^{Y}$ be defined with respect to a character $\chi$ of $E^{\times}$such that $\left.\chi\right|_{F^{\times}}=\epsilon_{E / F}^{2}$. Then

$$
\begin{aligned}
& \beta_{\mathcal{V}^{\prime}}^{Y}(g)=\chi((t-1) \delta), \\
& \alpha_{\mathcal{V}^{\prime}}(g)=1 .
\end{aligned}
$$

Proof. We will assume that $\epsilon=1$ in the proof. The case for $\epsilon=-1$ is analogous. Similar to the computation in Subsection 6.1, we have

$$
\begin{aligned}
\beta_{\mathcal{V}^{\prime}}^{Y}(g) & =\chi(x(g)) \chi(\delta)\left(\Delta, \operatorname{det}\left(\mathcal{V}^{\prime}\right) \Delta\right)_{F} \gamma_{F}\left(-\Delta, \psi_{0}\right)^{-2} \gamma_{F}\left(-1, \psi_{0}\right)^{2} \\
& =\chi(x(g) \delta)\left(\Delta, \operatorname{det}\left(\mathcal{V}^{\prime}\right) \Delta\right)_{F} \gamma_{F}\left(\Delta, \psi_{0}\right)^{-2} .
\end{aligned}
$$

We have $\left(\Delta, \operatorname{det}\left(\mathcal{V}^{\prime}\right) \Delta\right)_{F}=(\Delta,-1)_{F}=1$ and $\gamma_{F}\left(\Delta, \psi_{0}\right)^{-2}=(\Delta, \Delta)_{F}=1$ because $E$ is an unramified extension. Therefore,

$$
\beta_{\mathcal{V}^{\prime}}^{Y}(g)=\chi(x(g) \delta)=\chi\left(r^{-1}(t-1) \delta\right)=\chi((t-1) \delta)(\Delta, r)_{F}=\chi((t-1) \delta) .
$$

Suppose that $\epsilon=1$. Hence, $\mathcal{V}^{\prime}$ is a skew-hermitian space. Let $\left\{v_{1}^{\prime}, v_{2}^{\prime}\right\}$ be a basis of $\mathcal{V}^{\prime}$ such that $\mathbf{h}^{\prime}\left(v_{1}^{\prime}, v_{1}^{\prime}\right)=\mathbf{h}^{\prime}\left(v_{2}^{\prime}, v_{2}^{\prime}\right)=0$ and $\mathbf{h}^{\prime}\left(v_{1}^{\prime}, v_{2}^{\prime}\right)=\mathbf{h}^{\prime}\left(v_{2}^{\prime}, v_{1}^{\prime}\right)=$ $\delta$. Define $w_{1}:=-(2 \Delta r)^{-1}(v-\widehat{v}) \otimes v_{1}^{\prime}, w_{2}:=(2 \Delta r)^{-1} \delta(v-\widehat{v}) \otimes v_{1}^{\prime}, w_{3}:=$ $-(2 \Delta r)^{-1}(v-\widehat{v}) \otimes v_{2}^{\prime}, w_{4}:=(2 \Delta r)^{-1} \delta(v-\widehat{v}) \otimes v_{2}^{\prime}, w_{5}:=\delta(v+\widehat{v}) \otimes v_{2}^{\prime}$, 
$w_{6}:=(v+\widehat{v}) \otimes v_{2}^{\prime}, w_{7}:=\delta(v+\widehat{v}) \otimes v_{1}^{\prime}$, and $w_{8}:=(v+\widehat{v}) \otimes v_{1}^{\prime}$. Then it is clear that $\iota_{\mathcal{V}^{\prime}}(g)$ is equal to

$$
\frac{1}{2}\left[\begin{array}{cccccccc}
x+1 & -\Delta y & 0 & 0 & 0 & 0 & -2 \Delta^{2} r y & -2 \Delta r(x-1) \\
-y & x+1 & 0 & 0 & 0 & 0 & 2 \Delta r(x-1) & 2 \Delta r y \\
0 & 0 & x+1 & -\Delta y & -2 \Delta^{2} r y & -2 \Delta r(x-1) & 0 & 0 \\
0 & 0 & -y & x+1 & 2 \Delta r(x-1) & 2 \Delta r y & 0 & 0 \\
0 & 0 & -\frac{y}{2 \Delta r} & \frac{x-1}{2 \Delta r} & x+1 & y & 0 & 0 \\
0 & 0 & -\frac{x-1}{2 \Delta r} & \frac{y}{2 r} & \Delta y & x+1 & 0 & 0 \\
-\frac{y}{2 \Delta r} & \frac{x-1}{2 \Delta r} & 0 & 0 & 0 & 0 & x+1 & y \\
-\frac{x-1}{2 \Delta r} & \frac{y}{2 r} & 0 & 0 & 0 & 0 & \Delta y & x+1
\end{array}\right]
$$

with respect to the basis $\left\{w_{1}, \ldots, w_{8}\right\}$ of $\mathcal{W}+\overline{\mathcal{W}}$. Then we know that $X$ (resp. $Y$ ) is the $F$-space spanned by $\left\{w_{1}, \ldots, w_{4}\right\}$ (resp. $\left\{w_{5}, \ldots, w_{8}\right\}$ ). We know that $B^{*} / B=(\mathbf{l}+\overline{\mathbf{l}}) \otimes \mathbf{l}^{\prime *}+\left(\mathbf{l}^{*}+\overline{\mathbf{l}}^{*}\right) \otimes \mathbf{l}^{\prime}$ where $\overline{\mathbf{l}}:=\bar{L} / \varpi_{E} \bar{L}^{*}$ and $\overline{\mathbf{l}}^{*}:=\bar{L}^{*} / \bar{L}$. Therefore, it is clear that $B^{*} / B$ is either trivial or eightdimensional over $\mathbf{f}_{F}$. Suppose that $x \not \equiv 1 \bmod \mathfrak{p}_{F}$. We have the following two situations.

(i) Suppose that $B^{*}=B$. Obviously, we have $m\left(\overline{\iota \mathcal{V}^{\prime}(g)}\right)=1$ and $j\left(\overline{\iota_{\mathcal{V}^{\prime}}(g)}\right)$ $=0$. It is clear that Lemmas 5.5, 5.6, 5.9 can be extended to the case for $\mathcal{V}^{\prime}$ is two-dimensional i.e., (6.1.c) is also true for this case. Hence, we can conclude that $\alpha_{\mathcal{V}^{\prime}}(g)=1$.

(ii) Suppose that $B^{*} / B$ is eight-dimensional. Since $g \neq 1$, we know that $j\left(\overline{\iota \mathcal{V}^{\prime}(g)}\right)=4$ from the above matrix. Then it is clear that $x\left(\overline{\iota \mathcal{V}^{\prime}(g)}\right)$ is a square. So from (4.2.a), we see that $m\left(\overline{\left(\mathcal{V}^{\prime}(g)\right.}\right)=1$. Similar to the computation in Subsection 6.1, we conclude that $\alpha_{\mathcal{V}^{\prime}}(g)=1$ again.

Now we assume that $x \equiv 1 \bmod \mathfrak{p}_{F}$ and $g \neq 1$. In this case, it is clear that $\overline{\iota \mathcal{V}^{\prime}}(g)=1$. So $m\left(\overline{\iota \mathcal{V}^{\prime}(g)}\right)=1$ and $j\left(\overline{\iota_{\mathcal{V}^{\prime}}(g)}\right)=0$. Now we know that

$$
\begin{aligned}
& c=\frac{1}{4 \Delta r}\left[\begin{array}{cccc}
0 & 0 & -y & x-1 \\
0 & 0 & -(x-1) & \Delta y \\
-y & x-1 & 0 & 0 \\
-(x-1) & \Delta y & 0 & 0
\end{array}\right], \\
& d=\frac{1}{2}\left[\begin{array}{cccc}
x+1 & y & 0 & 0 \\
\Delta y & x+1 & 0 & 0 \\
0 & 0 & x+1 & y \\
0 & 0 & \Delta y & x+1
\end{array}\right] .
\end{aligned}
$$

Therefore,

$$
-d . c^{*}=\frac{1}{4 \Delta r}\left[\begin{array}{cccc}
0 & 0 & y & 0 \\
0 & 0 & 0 & -\Delta y \\
y & 0 & 0 & 0 \\
0 & -\Delta y & 0 & 0
\end{array}\right] \text {. }
$$


Now the self-dual lattice $A$ in $\mathcal{W}+\overline{\mathcal{W}}$ is of the form $L_{X}+L_{Y}$ where $L_{X}:=$ $\sum_{i=1}^{4} \mathfrak{p}_{F}^{\lambda_{i}} w_{i}$ and $L_{Y}:=\sum_{i=5}^{8} \mathfrak{p}_{F}^{\lambda_{F}-\lambda_{i-4}} w_{i}$ for some $\lambda_{1}, \ldots, \lambda_{4} \in \mathbf{Z}$. Again fix a Haar measure $\mu_{X}$ on $L_{X}$ such that $\mu_{X}\left(L_{X}\right)=q^{-\lambda_{0}}$ where $\lambda_{0}=-2 \lambda_{F}+$ $\sum_{i=1}^{4} \lambda_{i}$. For $x \in X$, write $x=\sum_{i=1}^{4} t_{i} w_{i}$. Then

$$
\psi\left(\frac{1}{2}\left\langle\left\langle-d . c^{*} \cdot x, x\right\rangle\right)=\psi\left(\frac{y}{4 \Delta r}\left(-t_{1} t_{3}+\Delta t_{2} t_{4}\right)\right)=\psi\left(\frac{-y}{4 \Delta r} t_{1} t_{3}\right) \psi\left(\frac{y}{4 r} t_{2} t_{4}\right)\right.
$$

from (6.2.b). Let $L_{X}[g]:=\sum_{i=1}^{4} \mathfrak{p}_{F}^{k_{i}} w_{i}$ for some $k_{i} \in \mathbf{Z}$ be as defined in (5.8.a). As in Subsection 5.9, we have

$$
\begin{aligned}
& \left(M_{Y}\left[\iota^{\prime}(g)\right] \cdot f_{Y}\right)(0) \\
& =|\operatorname{det}(c)|^{1 / 2} \mu_{X}\left(L_{X}\right) \sum_{t_{1} \in \mathfrak{p}_{F}^{k_{1}} / \mathfrak{p}_{F}^{\lambda_{1}}} \sum_{t_{2} \in \mathfrak{p}_{F}^{k_{2}} / \mathfrak{p}_{F}^{\lambda_{2}}} \sum_{t_{3} \in \mathfrak{p}_{F}^{k_{3}} / \mathfrak{p}_{F}^{\lambda_{3}}} \\
& \cdot \sum_{t_{4} \in \mathfrak{p}_{F}^{k_{4} / \mathfrak{p}_{F}^{\lambda_{4}}}} \psi\left(\frac{-y}{4 \Delta r} t_{1} t_{3}\right) \psi\left(\frac{y}{4 r} t_{2} t_{4}\right) .
\end{aligned}
$$

For a fixed $t_{3}, \psi\left(\frac{-y}{4 \Delta r} t_{1} t_{3}\right)$ is a character of the additive group $\mathfrak{p}_{F}^{k_{1}} / \mathfrak{p}_{F}^{\lambda_{1}}$. So $\sum_{t_{1} \in \mathfrak{p}_{F}^{k_{1}} / \mathfrak{p}_{F}^{\lambda_{1}}} \psi\left(\frac{-y}{4 \Delta r} t_{1} t_{3}\right)$ is not zero if and only if $\psi\left(\frac{-y}{4 \Delta r} t_{1} t_{3}\right)$ is the trivial character of $\mathfrak{p}_{F}^{k_{1}} / \mathfrak{p}_{F}^{\lambda_{1}}$. Hence,

$$
\left(M_{Y}\left[\mathcal{L}^{\prime}(g)\right] \cdot f_{Y}\right)(0)=|\operatorname{det}(c)|^{1 / 2} \mu_{X}\left(L_{X}\right) \sum_{t_{1} \in \mathfrak{p}_{F}^{k_{1}} / \mathfrak{p}_{F}^{\lambda_{1}}} \sum_{t_{2} \in \mathfrak{p}_{F}^{k_{2}} / \mathfrak{p}_{F}^{\lambda_{2}}} 1 .
$$

It is easy to check that $|\operatorname{det}(c)|^{1 / 2} q^{-\lambda_{0}} q^{\lambda_{1}-k_{1}} q^{\lambda_{2}-k_{2}}=1$. Hence, $\left(M_{Y}\left[\iota^{\prime}(g)\right] . f_{Y}\right)(0)=1$. Because now $m\left(\overline{\iota \mathcal{V}^{\prime}(g)}\right)=1$ and $j\left(\overline{\iota \mathcal{V}^{\prime}(g)}\right)=0$, we conclude that $\alpha_{\mathcal{V}^{\prime}}(g)=1$.

6.3. . In this subsection, we assume that $\mathcal{V}$ is one-dimensional and $\mathcal{V}^{\prime}$ is twodimensional anisotropic. We know that $\mathcal{V}^{\prime}$ has an $L^{\prime}$-admissible orthogonal decomposition $\mathcal{V}_{1}^{\prime} \oplus \mathcal{V}_{2}^{\prime}$ of nondegenerate one-dimensional subspaces. Let $\mathbf{h}^{\prime}$ be the form on $\mathcal{V}^{\prime}, \mathbf{h}_{1}^{\prime}:=\left.\mathbf{h}^{\prime}\right|_{\mathcal{V}_{1}^{\prime}}$ and $\mathbf{h}_{2}^{\prime}:=\left.\mathbf{h}^{\prime}\right|_{\mathcal{V}_{2}^{\prime}}$. Let $v_{i}^{\prime}$ be a nonzero element in $\mathcal{V}_{i}^{\prime}$. Define $r_{i}^{\prime}:=\mathbf{h}_{i}^{\prime}\left(v_{i}^{\prime}, v_{i}^{\prime}\right)$. Since $\mathcal{V}^{\prime}$ is anisotropic, we have $\left(\Delta,-r_{1}^{\prime} r_{2}^{\prime}\right)_{F}=-1$.

Lemma. Suppose that $\mathcal{V}$ is one-dimensional and $\mathcal{V}^{\prime}$ is two-dimensional anisotropic. Suppose that $g:=t=x+\delta y$ for $t \in E^{(1)}$ and $x, y \in \mathcal{O}_{F}$ is an element in $U(\mathcal{V})$ and $g \neq 1$. Let $\beta_{\mathcal{V}^{\prime}}^{Y}$ be defined with respect to a character $\chi$ of $E^{\times}$such that $\left.\chi\right|_{F^{\times}}=\epsilon_{E / F}^{2}$. Then

$$
\begin{aligned}
& \beta_{\mathcal{V}^{\prime}}^{Y}(g)=-\chi((t-1) \delta), \\
& \alpha_{\mathcal{V}^{\prime}}(g)= \begin{cases}\left(\frac{\overline{2 x-2}}{\mathbf{f}_{F}}\right), & \text { if } x \not \equiv 1 \bmod \mathfrak{p}_{F} \\
-1, & \text { if } x \equiv 1 \bmod \mathfrak{p}_{F}\end{cases}
\end{aligned}
$$


Proof. We know that $x(g)=(4 r)^{-1}(t-1)$ and $j(g)=1$. If $\epsilon=-1$, then we have

$$
\beta_{\mathcal{V}^{\prime}}^{Y}(g)=\chi(x(g))\left(\Delta, r_{1}^{\prime} r_{2}^{\prime}\right)_{F}(\Delta, \Delta)_{F}=-\chi((t-1) \delta)
$$

as in Subsection 6.2. If $\epsilon=1$, it is easy to check that (6.3.a) is also true.

It is clear that $\alpha_{\mathcal{V}^{\prime}}(g)=\alpha_{\mathcal{V}_{1}^{\prime}}(g) \alpha_{\mathcal{V}_{2}^{\prime}}(g)$. Let $\varrho_{i}$ be the $\varrho$ in Subsection 5.2 for the pair $\left(U(\mathcal{V}), U\left(\mathcal{V}_{i}^{\prime}\right)\right)$. It is clear that $\operatorname{ord}\left(\varrho_{1}\right)$ and $\operatorname{ord}\left(\varrho_{2}\right)$ have different parities since $\mathcal{V}^{\prime}$ is anisotropic and $E$ is unramified. Therefore, by Lemma 6.1, we get $\alpha_{\mathcal{V}^{\prime}}(g)=\left(\frac{\overline{2 x-2}}{\mathbf{f}_{F}}\right)$ if $x \not \equiv 1 \bmod \mathfrak{p}_{F}$. If $x \equiv 1 \bmod \mathfrak{p}_{F}$, then

$$
\begin{aligned}
\alpha \mathcal{V}^{\prime}(g) & =\gamma_{F}\left(-\Delta, \psi_{0}\right)^{2}\left(\Delta, 2 y \varrho_{1}\right)_{F}\left(\Delta, 2 y \varrho_{2}\right)_{F}\left(\left(\frac{-1}{\mathbf{f}_{F}}\right)^{\overline{\operatorname{ord}}\left(\psi_{0}\right)}\right)^{2} \\
& =(\Delta,-1)\left(\Delta, \varrho_{1} \varrho_{2}\right)=-1 .
\end{aligned}
$$

6.4. . In this subsection, we assume that $\mathcal{V}$ is two-dimensional isotropic and $\mathcal{V}^{\prime}$ is one-dimensional. Let $\left\{v_{1}, v_{2}\right\}$ be a standard $E$-basis of $\mathcal{V}$. Define $X:=E v_{1} \otimes \mathcal{V}^{\prime}$ and $Y:=E v_{2} \otimes \mathcal{V}^{\prime}$.

Lemma. Suppose that $\mathcal{V}$ is two-dimensional isotropic and $\mathcal{V}^{\prime}$ is one-dimensional. Suppose that $g:=\left[\begin{array}{cc}\tau(k) & 0 \\ 0 & k^{-1}\end{array}\right]$ for some $k \in \mathcal{O}_{E}^{\times}-\mathfrak{p}_{E}^{\times}$is an element of $U(\mathcal{V})$. Let $\beta_{\mathcal{V}^{\prime}}^{Y}$ be defined with respect to $\chi$, and $B:=B\left(L, L^{\prime}\right)$ is a good lattice in $\mathcal{W}$. Then

$$
\begin{aligned}
\beta_{\mathcal{V}^{\prime}}^{Y} & =\chi(\tau(k)), \\
\alpha_{\mathcal{V}^{\prime}}(g) & = \begin{cases}1, & \text { if } B^{*} / B \text { is trivial; } \\
\left(\frac{\overline{k \tau(k)}}{\mathbf{f}_{F}}\right), & \text { if } B^{*} / B \text { is four-dimensional. }\end{cases}
\end{aligned}
$$

Proof. From the assumption of $k$, we know that $g \in U(\mathcal{V})_{L}$. It is clear that $g$ stabilizes $E v_{1}$. So $j(g)=0$ and $x(g)=\tau(k)$. By [Kl] Theorem 3.1, it is clear that

$$
\beta_{\mathcal{V}^{\prime}}^{Y}(g)=\chi(x(g))=\chi(\tau(k)) .
$$

Let $v^{\prime}$ be a nonzero element in $\mathcal{V}^{\prime}$ and $r^{\prime}:=\mathbf{h}^{\prime}\left(v^{\prime}, v^{\prime}\right)$. We will choose $v^{\prime}$ such that $\operatorname{ord}\left(r^{\prime}\right)=0$ or 1 . Define $w_{1}:=\frac{-1}{\delta r^{\prime}} v_{1} \otimes v^{\prime}, w_{2}:=\frac{1}{r^{\prime}} v_{1} \otimes v^{\prime}$, $w_{3}:=\delta v_{2} \otimes v^{\prime}, w_{4}:=v_{2} \otimes v^{\prime}$. Then the set $\left\{w_{1}, w_{2}, w_{3}, w_{4}\right\}$ is a standard $F$ basis of $\mathcal{W}$. Then $X=F w_{1}+F w_{2}$ and $Y=F w_{3}+F w_{4}$. Write $k=x_{0}+\delta y_{0}$ for $x_{0}, y_{0} \in \mathcal{O}_{F}$. It is easy to check that

$$
\iota_{\mathcal{V}}^{\prime}(g)=\left[\begin{array}{cccc}
x_{0} & y_{0} & 0 & 0 \\
\Delta y_{0} & x_{0} & 0 & 0 \\
0 & 0 & \frac{x_{0}}{k \tau(k)} & \frac{-\Delta y_{0}}{k \tau(k)} \\
0 & 0 & \frac{-y_{0}}{k \tau(k)} & \frac{x_{0}}{k \tau(k)}
\end{array}\right] \in \operatorname{Sp}(\mathcal{W})
$$


with respect to the basis $\left\{w_{1}, \ldots, w_{4}\right\}$. Then (2.2.b) for $f=f_{Y}$ and $y=0$ becomes

$$
\left(M_{Y}\left[\iota \mathcal{V}^{\prime}(g)\right] . f_{Y}\right)(0)=\left|x_{0}^{2}-\Delta y_{0}^{2}\right|^{1 / 2} f_{Y}(0) .
$$

We know that $\left|x_{0}^{2}-\Delta y_{0}^{2}\right|=1$ because we assume that $k \in \mathcal{O}_{E}^{\times}-\mathfrak{p}_{E}^{\times}$. Hence we have

$$
\left(M_{Y}\left[\iota^{\prime}(g)\right] . f_{Y}\right)(0)=1 .
$$

Now $\mathcal{V}$ has two good lattices (up to equivalence), namely $\mathcal{O}_{E} v_{1}+\mathcal{O}_{E} v_{2}$ and $\mathcal{O}_{E} v_{1}+\mathfrak{p}_{E} v_{2}$. There is only one good lattice in $\mathcal{V}^{\prime}$, namely $\mathcal{O}_{E} v^{\prime}$. First, suppose that $L=\mathcal{O}_{E} v_{1}+\mathcal{O}_{E} v_{2}$. Then,

$$
\begin{aligned}
B:=B\left(L, L^{\prime}\right) & =\mathcal{O}_{E} v_{1} \otimes v^{\prime}+\mathcal{O}_{E} v_{2} \otimes v^{\prime} \\
& =\mathcal{O}_{F} v_{1} \otimes v^{\prime}+\mathcal{O}_{F} \delta v_{1} \otimes v^{\prime}+\mathcal{O}_{F} v_{2} \otimes v^{\prime}+\mathcal{O}_{F} \delta v_{2} \otimes v^{\prime}
\end{aligned}
$$

Next, suppose that $L=\mathcal{O}_{E} v_{1}+\mathfrak{p}_{E} v_{2}$. Then,

$$
\begin{aligned}
B & =\mathcal{O}_{E} v_{1} \otimes v^{\prime}+\mathfrak{p}_{E} v_{2} \otimes v^{\prime} \\
& =\mathcal{O}_{F} v_{1} \otimes v^{\prime}+\mathcal{O}_{F} \delta v_{1} \otimes v^{\prime}+\mathfrak{p}_{F} v_{2} \otimes v^{\prime}+\mathfrak{p}_{F} \delta v_{2} \otimes v^{\prime} .
\end{aligned}
$$

Let $L_{X}:=\mathcal{O}_{F} w_{1}+\mathcal{O}_{F} w_{2}, L_{Y}:=\mathcal{O}_{F} w_{3}+\mathcal{O}_{F} w_{4}$, and $A:=L_{X}+L_{Y}$. Then, $A$ is a self-dual lattice in $\mathcal{W}$ and $B \subseteq A \subseteq B^{*}$. It is easy to see that $B^{*} / B$ is either trivial or four-dimensional. Analogous to Lemma 5.5, we can show that

$$
\left(\Xi \circ M_{A}\left[\iota_{\mathcal{V}^{\prime}}(g)\right] . f_{A}\right)(0)=\mu_{X}\left(L_{X}\right) \mu\left(\iota \mathcal{V}^{\prime}(g) \cdot A \cap A\right) .
$$

Because we assume that $k \in \mathcal{O}_{E}-\mathfrak{p}_{E}$, we know that $\iota \mathcal{V}^{\prime}(g)$ stabilizes the lattice $A$. So we get

$$
\left(\Xi \circ M_{A}\left[\iota \mathcal{V}^{\prime}(g)\right] . f_{A}\right)(0)=\mu_{X}\left(L_{X}\right) .
$$

Analogous to Lemma 5.6, we can show that $\Xi . f_{A}=\mu_{X}\left(L_{X}\right) f_{Y}$. Hence from (4.4.c), (6.4.c) and (6.4.d), we have

$$
\alpha \mathcal{V}^{\prime}(g) m\left(\overline{\iota \mathcal{V}^{\prime}(g)}\right)=1 .
$$

Now we have the following two possibilities. If $B^{*}=B$, then it is clear that $m\left(\overline{\left(\mathcal{V}^{\prime}(g)\right.}\right)=1$ and $j\left(\overline{\iota_{\mathcal{V}^{\prime}}(g)}\right)=0$. Therefore, we have $\alpha_{\mathcal{V}^{\prime}}(g)=1$. If $B^{*} / B$ is four-dimensional, by (6.4.b) we have $j\left(\overline{\iota^{\prime}(g)}\right)=0$ and $m\left(\overline{\left(\mathcal{L}^{\prime}(g)\right.}\right)=$ $\left(\frac{x \overline{\left(\overline{\mathcal{L}^{\prime}}(g)\right)}}{\mathbf{f}_{F}}\right)=\left(\frac{\overline{x_{0}^{2}-\Delta y_{0}^{2}}}{\mathbf{f}_{F}}\right)=\left(\frac{\overline{k \tau(k)}}{\mathbf{f}_{F}}\right)$. Hence, $\alpha_{\mathcal{V}^{\prime}}(g)=\left(\frac{\overline{k \tau(k)}}{\mathbf{f}_{F}}\right)$.

6.5. . In this subsection, we assume that both $\mathcal{V}$ and $\mathcal{V}^{\prime}$ are two-dimensional and isotropic. Let $\left\{v_{1}, v_{2}\right\}$ (resp. $\left\{v_{1}^{\prime}, v_{2}^{\prime}\right\}$ ) be a standard basis of $\mathcal{V}$ (resp. $\mathcal{V}^{\prime}$ ). As in Subsection 6.4, let $X:=E v_{1} \otimes \mathcal{V}^{\prime}$ and $Y:=E v_{2} \otimes \mathcal{V}^{\prime}$. 
Lemma. Suppose that both $\mathcal{V}$ and $\mathcal{V}^{\prime}$ are two-dimensional and isotropic. Let $\beta_{\mathcal{V}^{\prime}}^{Y}$ be defined with respect to $\chi$, and $g:=\left[\begin{array}{cc}\tau(k) & 0 \\ 0 & k^{-1}\end{array}\right] \in U(\mathcal{V})$ for some $k \in \mathcal{O}_{E}^{\times}-\mathfrak{p}_{E}^{\times}$. Then

$$
\begin{aligned}
& \beta_{\mathcal{V}^{\prime}}^{Y}(g)=\chi(\tau(k)), \\
& \alpha_{\mathcal{V}^{\prime}}(g)=1 .
\end{aligned}
$$

Proof. It is clear that $x(g)=\tau(k)$ and $j(g)=0$. Similar to the computation in Subsection 6.4, we have $\beta_{\mathcal{V}^{\prime}}^{Y}(g)=\chi(x(g))$.

Suppose that $\epsilon=1$. Define $w_{1}:=v_{1} \otimes v_{1}^{\prime}, w_{2}:=-\frac{\delta}{\Delta} v_{1} \otimes v_{1}^{\prime}, w_{3}:=-v_{1} \otimes v_{2}^{\prime}$, $w_{4}:=\frac{\delta}{\Delta} v_{1} \otimes v_{2}^{\prime}, w_{5}:=v_{2} \otimes v_{2}^{\prime}, w_{6}:=\delta v_{2} \otimes v_{2}^{\prime}, w_{7}:=v_{2} \otimes v_{1}^{\prime}, w_{8}:=\delta v_{2} \otimes v_{1}^{\prime}$. Then the set $\left\{w_{1}, \ldots, w_{8}\right\}$ is a standard basis of $\mathcal{W}$. Write $k=x_{0}+\delta y_{0}$ for $x_{0}, y_{0} \in F$. Then it is clear that

$$
\iota_{\mathcal{V}^{\prime}}(g)=\left[\begin{array}{cccccccc}
x_{0} & y_{0} & 0 & 0 & 0 & 0 & 0 & 0 \\
\Delta y_{0} & x_{0} & 0 & 0 & 0 & 0 & 0 & 0 \\
0 & 0 & x_{0} & y_{0} & 0 & 0 & 0 & 0 \\
0 & 0 & \Delta y_{0} & x_{0} & 0 & 0 & 0 & 0 \\
0 & 0 & 0 & 0 & \frac{x_{0}}{k \tau(k)} & \frac{-\Delta y_{0}}{k \tau(k)} & 0 & 0 \\
0 & 0 & 0 & 0 & \frac{-y_{0}}{k \tau(k)} & \frac{x_{0}}{k \tau(k)} & 0 & 0 \\
0 & 0 & 0 & 0 & 0 & 0 & \frac{x_{0}}{k \tau(k)} & \frac{-\Delta y_{0}}{k \tau(k)} \\
0 & 0 & 0 & 0 & 0 & 0 & \frac{-y_{0}}{k \tau(k)} & \frac{x_{0}}{k \tau(k)}
\end{array}\right] \in \operatorname{Sp}(\mathcal{W})
$$

with respect to the basis $\left\{w_{1}, \ldots, w_{8}\right\}$ of $\mathcal{W}$. Then $X$ (resp. $Y$ ) is the $F$ space spanned by $\left\{w_{1}, \ldots, w_{4}\right\}$ (resp. $\left\{w_{5}, \ldots, w_{8}\right\}$ ). We can see that $B^{*} / B$ is either trivial or eight-dimensional. From (6.4.b), it is clear that $j\left(\overline{{\iota \mathcal{V}^{\prime}}^{\prime}(g)}\right)=$ 0 and $x\left(\overline{\iota \mathcal{V}^{\prime}(g)}\right)$ is a square in $\mathbf{f}_{F}$ for both cases. Hence, $m\left(\overline{\left(\mathcal{V}^{\prime}(g)\right.}\right)=1$ from (4.2.a). We can also show that (6.4.e) is also true for this case. Therefore, we have $\alpha_{\mathcal{V}^{\prime}}(g)=1$.

It is clear that by the same argument, we can prove that $\alpha_{\mathcal{V}^{\prime}}(g)=1$ when $\epsilon=-1$.

6.6. . In this subsection, we assume that $\mathcal{V}$ is two-dimensional isotropic and $\mathcal{V}^{\prime}$ is two-dimensional anisotropic. Let $\mathcal{V}_{i}^{\prime}, r_{i}^{\prime}$ be as defined in Subsection 6.3.

Lemma. Suppose that $\mathcal{V}$ is two-dimensional isotropic and $\mathcal{V}^{\prime}$ is two-dimensional anisotropic. Let $\beta_{\mathcal{V}^{\prime}}^{Y}$ be defined with respect to $\chi$, and $g:=\left[\begin{array}{cc}\tau(k) & 0 \\ 0 & k^{-1}\end{array}\right]$ $\in U(\mathcal{V})$ for some $k \in \mathcal{O}_{E}^{\times}-\mathfrak{p}_{E}^{\times}$. Then

$$
\begin{aligned}
& \beta_{\mathcal{V}^{\prime}}^{Y}(g)=\chi(\tau(k)), \\
& \alpha_{\mathcal{V}^{\prime}}(g)=\left(\frac{\overline{k \tau(k)}}{\mathbf{f}_{F}}\right) .
\end{aligned}
$$


Proof. The computation for $\beta_{\mathcal{V}^{\prime}}^{Y}(g)$ is as in Subsection 6.5. As in Subsection $6.3, \alpha_{\mathcal{V}^{\prime}}(g)=\alpha_{\mathcal{V}_{1}^{\prime}}(g) \alpha_{\mathcal{V}_{2}^{\prime}}(g)$. Define $L_{i}^{\prime}:=L^{\prime} \cap \mathcal{V}_{i}^{\prime}$ and $B_{i}:=B\left(L, L_{i}^{\prime}\right)$. It is clear that one of $B_{1}^{*} / B_{1}, B_{2}^{*} / B_{2}$ is trivial and the other is four-dimensional. Hence by Lemma 6.4, we get $\alpha_{\mathcal{V}^{\prime}}(g)=\left(\frac{\overline{k \tau(k)}}{\mathbf{f}_{F}}\right)$.

\subsection{Proof of Proposition 3.3 for $\mathcal{V} \epsilon$-hermitian and $E$ unramified.} Let $g$ be a representative of a nontrivial $\left[U(\mathcal{V})_{L}, U(\mathcal{V})_{L}\right]$-coset in $S U(\mathcal{V})_{L}$. As in Subsection 1.3, we have an $L^{\prime}$-admissible decomposition $\mathcal{V}^{\prime}=\bigoplus_{i} \mathcal{V}_{i}^{\prime}$ of nondegenerate subspaces such that each $\mathcal{V}_{i}$ is either one-dimensional or a hyperbolic plane. Let $Y_{i}:=Y^{\circ} \otimes \mathcal{V}_{i}^{\prime}$. Then as explained in Subsection 3.6, we have

$$
\alpha_{\mathcal{V}^{\prime}}(g) \beta_{\mathcal{V}^{\prime}}^{Y}(g)=\prod_{i} \alpha_{\mathcal{V}_{i}^{\prime}}(g) \beta_{\mathcal{V}_{i}^{\prime}}^{Y_{i}}(g)
$$

if we choose characters $\chi_{i}$ and $\chi$ properly. Therefore, we reduce the problem to the computation of each $\alpha_{\mathcal{V}_{i}^{\prime}}(g)$ and $\beta_{\mathcal{V}_{i}^{\prime}}^{Y_{i}}(g)$. As described in Subsection 1.3, we also have an $L$-admissible decomposition $\mathcal{V}=\mathcal{V}_{1} \oplus \mathcal{V}_{2}$ of nondegenerate subspaces such that $L \cap \mathcal{V}_{1}=L^{*} \cap \mathcal{V}_{1}$ and $L \cap \mathcal{V}_{2}=\varpi_{E}\left(L^{*} \cap \mathcal{V}_{2}\right)$. We should note here that $\mathcal{V}_{1}, \mathcal{V}_{2}$ can not have two-dimensional anisotropic subspace. We know that the quotient $S U(\mathcal{V})_{L} /\left[U(\mathcal{V})_{L}, U(\mathcal{V})_{L}\right]$ is nontrivial if and only if both $\mathbf{l}, \mathbf{l}^{*}$ are nontrivial. We know that $\operatorname{dim}(\mathbf{l})=\operatorname{dim}\left(\mathcal{V}_{1}\right)$ and $\operatorname{dim}\left(\mathbf{l}^{*}\right)=\operatorname{dim}\left(\mathcal{V}_{2}\right)$. So we just need to consider the case that both $\mathcal{V}_{1}, \mathcal{V}_{2}$ are nontrivial.

Suppose that both $\mathcal{V}_{1}, \mathcal{V}_{2}$ are even-dimensional. Then, $\mathcal{V}_{1}, \mathcal{V}_{2}$ are direct sum of hyperbolic planes. Choose $L$-admissible hyperbolic planes $\mathcal{V}_{1}^{0}, \mathcal{V}_{2}^{0}$ in $\mathcal{V}_{1}, \mathcal{V}_{2}$ respectively. Let $g_{1}:=\left[\begin{array}{cc}\tau(k) & 0 \\ 0 & k^{-1}\end{array}\right] \in U\left(\mathcal{V}_{1}^{0}\right) \subseteq U\left(\mathcal{V}_{1}\right) \subseteq U(\mathcal{V})$ and $g_{2}:=\left[\begin{array}{cc}\tau(k)^{-1} & 0 \\ 0 & k\end{array}\right] \in U\left(\mathcal{V}_{2}^{0}\right) \subseteq U\left(\mathcal{V}_{2}\right) \subseteq U(\mathcal{V})$ for some $k \in \mathcal{O}_{E}^{\times}-\mathfrak{p}_{E}^{\times}$. Then $g:=g_{1} g_{2}$ is an element in $S U(\mathcal{V})_{L}$ and the set $\left\{g_{1} g_{2} \mid k \in \mathcal{O}_{E}^{\times}-\mathfrak{p}_{E}^{\times}\right\}$ contains a set of representatives of $\left[U(\mathcal{V})_{L}, U(\mathcal{V})_{L}\right]$-cosets in $S U(\mathcal{V})_{L}$. From Lemmas 6.4 and 6.5 , we see that

$$
\beta_{\mathcal{V}_{i}^{\prime}}^{Y_{i}}\left(g_{1}\right) \beta_{\mathcal{V}_{i}^{\prime}}^{Y_{i}}\left(g_{2}\right)=\chi_{i}(\tau(k)) \chi_{i}\left(\tau(k)^{-1}\right)=1 .
$$

Let $L_{j}:=L \cap \mathcal{V}_{j}^{0}, L_{i}^{\prime}:=L^{\prime} \cap \mathcal{V}_{i}^{\prime}$, and $B_{j, i}:=B\left(L_{j}, L_{i}^{\prime}\right)$. If $\mathcal{V}_{i}^{\prime}$ is onedimensional, then it is easy to check that one of $B_{1, i}^{*} / B_{1, i}, B_{2, i}^{*} / B_{2, i}$ is trivial and the other is four-dimensional. If $\mathcal{V}_{i}^{\prime}$ is a hyperbolic plane, then one of $B_{1, i}^{*} / B_{1, i}, B_{2, i}^{*} / B_{2, i}$ is trivial and the other is eight-dimensional. Therefore, by Lemmas 6.4 and 6.5 , we get

$$
\alpha_{\mathcal{V}_{i}^{\prime}}\left(g_{1}\right) \alpha_{\mathcal{V}_{i}^{\prime}}\left(g_{2}\right)= \begin{cases}\left(\frac{\overline{k \tau(k)}}{\mathbf{f}_{F}}\right), & \text { if } \mathcal{V}_{i}^{\prime} \text { is one-dimensional; } \\ 1, & \text { if } \mathcal{V}_{i}^{\prime} \text { is a hyperbolic plane. }\end{cases}
$$


Hence from (6.7.b) and (6.7.c), we have

$$
\begin{aligned}
\alpha_{\mathcal{V}_{i}^{\prime}}(g) \beta_{\mathcal{V}_{i}^{\prime}}^{Y}(g) & =\alpha_{\mathcal{V}_{i}^{\prime}}\left(g_{1}\right) \alpha_{\mathcal{V}_{i}^{\prime}}\left(g_{2}\right) \beta_{\mathcal{V}_{i}^{\prime}}^{Y_{i}}\left(g_{1}\right) \beta_{\mathcal{V}_{i}^{\prime}}^{Y_{i}}\left(g_{2}\right) \\
& = \begin{cases}\left(\frac{\overline{k \tau(k)}}{\mathbf{f}_{F}}\right), & \text { if } \mathcal{V}_{i}^{\prime} \text { is one-dimensional; } \\
1, & \text { if } \mathcal{V}_{i}^{\prime} \text { is a hyperbolic plane. }\end{cases}
\end{aligned}
$$

If $\mathcal{V}^{\prime}$ is odd-dimensional, then only one of these $\mathcal{V}_{i}^{\prime}$ is one-dimensional and the others are hyperbolic planes. If $\mathcal{V}^{\prime}$ is even-dimensional, then there are either two or none of these $\mathcal{V}_{i}^{\prime}$ are one-dimensional and all others are hyperbolic planes. Therefore, we have

$$
\alpha \mathcal{V}^{\prime}(g) \beta_{\mathcal{V}^{\prime}}^{Y}(g)= \begin{cases}\left(\frac{\overline{k \tau(k)}}{\mathbf{f}_{F}}\right), & \text { if } \mathcal{V}^{\prime} \text { is odd-dimensional } \\ 1, & \text { if } \mathcal{V}^{\prime} \text { is even-dimensional. }\end{cases}
$$

Suppose that one of $\mathcal{V}_{1}, \mathcal{V}_{2}$ is odd-dimensional and the other is evendimensional. Without loss of generality, we may assume that $\mathcal{V}_{1}$ is odddimensional and $\mathcal{V}_{2}$ is even-dimensional. Let $\mathcal{V}_{1}^{0}$ be an $L$-admissible one dimensional nondegenerate subspace of $\mathcal{V}_{1}$. Let $\mathcal{V}_{2}^{0}$ be an $L$-admissible hyperbolic plane of $\mathcal{V}_{2}$. Define $g_{1}:=k \tau(k)^{-1} \in U\left(\mathcal{V}_{1}^{0}\right) \subseteq U\left(\mathcal{V}_{1}\right) \subseteq U(\mathcal{V})$ and $g_{2}:=\left[\begin{array}{cc}\tau(k) & 0 \\ 0 & k^{-1}\end{array}\right] \in U\left(\mathcal{V}_{2}^{0}\right) \subseteq U\left(\mathcal{V}_{2}\right) \subseteq U(\mathcal{V})$. We assume that $k \in \mathcal{O}_{E}-\mathfrak{p}_{E}$ and $k \tau\left(k^{-1}\right) \neq 1$. Therefore, $g:=g_{1} g_{2} \in S U(\mathcal{V})_{L}$ is a representative of a nontrivial $\left[U(\mathcal{V})_{L}, U(\mathcal{V})_{L}\right]$-coset in $S U(\mathcal{V})_{L}$. Let $L_{j}:=L \cap \mathcal{V}_{j}^{0}$, $L_{i}^{\prime}:=L^{\prime} \cap \mathcal{V}_{i}^{\prime}, B_{1, i}:=B\left(L_{1}+\bar{L}_{1}, L_{i}^{\prime}\right)$, and $B_{2, i}:=B\left(L_{2}, L_{i}^{\prime}\right)$. Write $k:=x_{0}+\delta y_{0}$ and $k \tau(k)^{-1}:=x+\delta y$ for $x_{0}, y_{0}, x, y \in \mathcal{O}_{F}$. Because $k \tau(k)^{-1}=\left(x_{0}+\delta y_{0}\right)\left(x_{0}-\delta y_{0}\right)^{-1}=\left(x_{0}^{2}+\Delta y_{0}^{2}+2 x_{0} y_{0} \delta\right)\left(x_{0}^{2}-\Delta y_{0}^{2}\right)^{-1}$, we have $x=\left(x_{0}^{2}+\Delta y_{0}^{2}\right)\left(x_{0}^{2}-\Delta y_{0}^{2}\right)^{-1}$. Since we assume that $k \tau(k)^{-1} \neq 1$, we have $y_{0} \neq 0$. We shall only consider the case that $x \not \equiv 1 \bmod \mathfrak{p}_{F}$, so we assume that $\operatorname{ord}\left(y_{0}\right)=0$. By Lemmas 6.1, 6.2, 6.4, 6.5, we have

$$
\begin{aligned}
& \alpha_{\mathcal{V}_{i}^{\prime}}\left(g_{1}\right) \beta_{\mathcal{V}_{i}^{\prime}}^{Y_{i}}\left(g_{1}\right)= \begin{cases}\chi_{i}\left(\left(k \tau(k)^{-1}-1\right) \delta\right), & \text { if } \operatorname{dim}\left(\mathcal{V}_{i}^{\prime}\right)=1 \text { and } \\
-\left(\frac{2 x-2}{\mathbf{f}_{F}}\right) \chi_{i}\left(\left(k \tau(k)^{-1}-1\right) \delta\right), & \text { if } \operatorname{dim}\left(B_{1, i}^{*} / B_{1, i}\right)=0 ; \\
\chi_{i}\left(\left(k \tau(k)^{-1}-1\right) \delta\right), & \operatorname{dim}\left(B_{1, i}^{*} / B_{1, i}\right)=4 ;\end{cases} \\
& \alpha_{\mathcal{V}_{i}^{\prime}}\left(g_{2}\right) \beta_{\mathcal{V}_{i}^{\prime}}^{Y_{i}}\left(g_{2}\right)= \begin{cases}\chi_{i}(\tau(k)), & \text { if } \operatorname{dim}\left(\mathcal{V}_{i}^{\prime}\right)=2 . \\
\left(\frac{\overline{k \tau(k)}}{\mathbf{f}_{F}}\right) \chi_{i}(\tau(k)), & \operatorname{dim}\left(\mathcal{V}_{i}^{\prime}\right)=1 \text { and } \operatorname{dim}\left(\mathcal{V}_{i}^{\prime}\right)=1 \text { and } \\
\chi_{i}(\tau(k)), & \operatorname{dim}\left(B_{2, i}^{*} / B_{2, i}\right)=4 ;\end{cases} \\
& \text { if } \operatorname{dim}\left(\mathcal{V}_{i}^{\prime}\right)=2 .
\end{aligned}
$$


We have

$$
\begin{aligned}
-\left(\frac{\overline{2 x-2}}{\mathbf{f}_{F}}\right) & =-\left(\frac{\overline{2\left(x_{0}^{2}+\Delta y_{0}^{2}\right)\left(x_{0}^{2}-\Delta y_{0}^{2}\right)^{-1}-2}}{\mathbf{f}_{F}}\right) \\
& =-\left(\frac{\overline{4 \Delta y_{0}^{2}\left(x_{0}^{2}-\Delta y_{0}^{2}\right)^{-1}}}{\mathbf{f}_{F}}\right)=-\left(\frac{\bar{\Delta}}{\mathbf{f}_{F}}\right)\left(\frac{\overline{x_{0}^{2}-\Delta y_{0}^{2}}}{\mathbf{f}_{F}}\right) .
\end{aligned}
$$

Because $\Delta$ is not a square in $F$, we have $\left(\frac{\bar{\Delta}}{\mathbf{f}_{F}}\right)=-1$. Therefore,

$$
-\left(\frac{\overline{2 x-2}}{\mathbf{f}_{F}}\right)=\left(\frac{\overline{x_{0}^{2}-\Delta y_{0}^{2}}}{\mathbf{f}_{F}}\right)=\left(\frac{\overline{k \tau(k)}}{\mathbf{f}_{F}}\right) .
$$

If $\mathcal{V}_{i}^{\prime}$ is one-dimensional, we know that one of $B_{1, i}^{*} / B_{1, i}, B_{2, i}^{*} / B_{2, i}$ is trivial and the other is four-dimensional. Therefore, we get

$$
\begin{aligned}
& \alpha_{\mathcal{V}_{i}^{\prime}}(g) \beta_{\mathcal{V}_{i}^{\prime}}^{Y_{i}}(g) \\
& = \begin{cases}\left(\frac{k \tau(k)}{\mathbf{f}_{F}}\right) \chi_{i}\left(\left(k \tau(k)^{-1}-1\right) \delta\right) \chi(\tau(k)), & \text { if } \mathcal{V}_{i}^{\prime} \text { is one-dimensional; } \\
\chi_{i}\left(\left(k \tau(k)^{-1}-1\right) \delta\right) \chi(\tau(k)), & \text { if } \mathcal{V}_{i}^{\prime} \text { is a hyperbolic plane. }\end{cases}
\end{aligned}
$$

We know

$$
\chi_{i}\left(\left(k \tau(k)^{-1}-1\right) \delta\right) \chi(\tau(k))=\chi_{i}((k-\tau(k)) \delta)=\chi_{i}\left(2 y_{0} \Delta\right)=\left(\Delta, 2 y_{0} \Delta\right)_{F}=1
$$

because $E$ is an unramified quadratic extension and $\operatorname{ord}\left(y_{0}\right)=0$. If $\mathcal{V}^{\prime}$ is even-dimensional, then there are either two or none of $\mathcal{V}_{i}^{\prime}$ are one-dimensional and all others are hyperbolic planes. If $\mathcal{V}^{\prime}$ is odd-dimensional, then exactly one of $\mathcal{V}_{i}^{\prime}$ is one-dimensional and the others are hyperbolic planes. Therefore, we have

$$
\alpha \mathcal{V}^{\prime}(g) \beta_{\mathcal{V}^{\prime}}^{Y}(g)= \begin{cases}\left(\frac{\overline{k \tau(k)}}{\mathbf{f}_{F}}\right), & \text { if } \mathcal{V}^{\prime} \text { is odd-dimensional } \\ 1, & \text { if } \mathcal{V}^{\prime} \text { is even-dimensional }\end{cases}
$$

Suppose that both $\mathcal{V}_{1}, \mathcal{V}_{2}$ are odd-dimensional. Let $\mathcal{V}_{1}^{0}$ (resp. $\mathcal{V}_{2}^{0}$ ) be an $L$-admissible one dimensional nondegenerate subspace of $\mathcal{V}_{1}$ (resp. $\left.\mathcal{V}_{2}\right)$. Let $g_{1}:=t=x+\delta y \in U\left(\mathcal{V}_{1}^{0}\right) \subseteq U\left(\mathcal{V}_{1}\right) \subset U(\mathcal{V})$ and $g_{2}:=t^{-1} \in U\left(\mathcal{V}_{2}^{0}\right) \subseteq$ $U\left(\mathcal{V}_{2}\right) \subset U(\mathcal{V})$ for some $t \in E^{(1)}$ and $t \notin 1+\mathfrak{p}_{E}$. Define $g:=\left(g_{1}, g_{2}\right) \in$ $U\left(\mathcal{V}_{1}\right) \times U\left(\mathcal{V}_{2}\right) \subset U(\mathcal{V})$. Hence, $g$ belongs to $S U(\mathcal{V})_{L}$ and is a representative of a nontrivial $\left[U(\mathcal{V})_{L}, U(\mathcal{V})_{L}\right]$-coset in $S U(\mathcal{V})_{L}$. Since $t \tau(t)=1$, we know that $t^{-1}=\tau(t)=x-\delta y$. Let $L_{j}:=L \cap \mathcal{V}_{j}^{0}, L_{i}^{\prime}:=L^{\prime} \cap \mathcal{V}_{i}^{\prime}$, and $B_{j, i}:=$ $B\left(L_{j}+\bar{L}_{j}, L_{i}^{\prime}\right)$. Therefore, by Lemmas 6.1, 6.2, 6.4, 6.5, we have

$$
\alpha_{\mathcal{V}_{i}^{\prime}}\left(g_{1}\right) \beta_{\mathcal{V}_{i}^{\prime}}^{Y_{i}}\left(g_{1}\right)= \begin{cases}\chi_{i}((t-1) \delta), & \text { if } \operatorname{dim}\left(\mathcal{V}_{i}^{\prime}\right)=1 \text { and } \\ -\left(\frac{\overline{2 x-2}}{\mathbf{f}_{F}}\right) \chi_{i}((t-1) \delta), & \text { if } \operatorname{dim}\left(B_{1, i}^{*} / B_{1, i}^{\prime}\right)=1 \text { and } \\ & \operatorname{dim}\left(B_{1, i}^{*} / B_{1, i}\right)=4 ; \\ \chi_{i}((t-1) \delta), & \text { if } \operatorname{dim}\left(\mathcal{V}_{i}^{\prime}\right)=2 .\end{cases}
$$


If $\mathcal{V}_{i}^{\prime}$ is one-dimensional, we know that one of $B_{1, i}^{*} / B_{1, i}, B_{2, i}^{*} / B_{2, i}$ is trivial and the other is four-dimensional. It is easy to check that $\chi_{i}\left((t-1)\left(t^{-1}-1\right) \delta^{2}\right)=$ $\chi_{i}((2-2 x) \Delta)=(\Delta,(2-2 x) \Delta)_{F}=1$ because $E$ is an unramified quadratic extension. Therefore

$$
\alpha \mathcal{V}_{i}^{\prime}(g) \beta_{\mathcal{V}_{i}^{\prime}}^{Y_{i}}(g)= \begin{cases}-\left(\frac{\overline{2 x-2}}{\mathbf{f}_{F}}\right), & \text { if } \mathcal{V}_{i}^{\prime} \text { is one-dimensional; } \\ 1, & \text { if } \mathcal{V}_{i}^{\prime} \text { is a hyperbolic plane. }\end{cases}
$$

If $\mathcal{V}^{\prime}$ is even-dimensional, then there are either two or none of $\mathcal{V}_{i}^{\prime}$ are onedimensional and all others are hyperbolic planes. If $\mathcal{V}^{\prime}$ is odd-dimensional, then only one of $\mathcal{V}_{i}^{\prime}$ is one-dimensional and the others are hyperbolic planes. We can write $t=k \tau(k)^{-1}$ for some $k$. Therefore, we have

$$
\alpha \mathcal{V}^{\prime}(g) \beta_{\mathcal{V}^{\prime}}^{Y}(g)= \begin{cases}\left(\frac{\overline{k \tau(k)}}{\mathbf{f}_{F}}\right), & \text { if } \mathcal{V}^{\prime} \text { is odd-dimensional; } \\ 1, & \text { if } \mathcal{V}^{\prime} \text { is even-dimensional. }\end{cases}
$$

Finally from (6.7.d), (6.7.f) and (6.7.g), we conclude that

$$
\alpha \mathcal{V}^{\prime}(g) \beta_{\mathcal{V}^{\prime}}^{Y}(g)= \begin{cases}\left(\frac{\overline{k \tau(k)}}{\mathbf{f}_{F}}\right), & \text { if } \mathcal{V}^{\prime} \text { is odd-dimensional; } \\ 1, & \text { if } \mathcal{V}^{\prime} \text { is even-dimensional }\end{cases}
$$

for $g$ in a set of representatives of nontrivial $\left[U(\mathcal{V})_{L}, U(\mathcal{V})_{L}\right]$-cosets in $S U(\mathcal{V})_{L}$. It is easy to check that the left-hand side of (6.7.h) is exactly the character $\zeta_{\mathcal{V}^{\prime}}$ defined in Subsection 3.3. Hence, we conclude $\alpha_{\mathcal{V}^{\prime}}(g) \beta_{\mathcal{V}^{\prime}}^{Y}(g)=$ $\zeta_{\mathcal{V}^{\prime}}(g)$ for any $g \in S U(\mathcal{V})_{L}$.

\section{Proof of Proposition 3.3 for unitary groups: Part II.}

In this section, we assume that $D=E$ is a ramified quadratic extension of $F$. Let $E:=F(\delta)$ where $\delta^{2}=\Delta$ is a nonsquare element in $F^{\times}$such that $\operatorname{ord}(\Delta)=1$. Without loss of generality, we assume that $\Delta:=\varpi_{E}$. As usual, fix a nontrivial character $\psi$ of $F$. Subsections 7.1-7.6 are parallel to Subsections 6.1-6.6. So these subsections will be sketchy in many places. In Subsections 7.7, 7.8 and 7.10, we also assume that $\#\left(\mathbf{f}_{F}\right)=3$.

7.1. . In this subsection, we keep the same assumption in Subsection 6.1 except that we assume that $E$ is a ramified extension.

Lemma. Suppose that both $\mathcal{V}$ and $\mathcal{V}^{\prime}$ are one-dimensional. Let $g \in U(\mathcal{V})$. Write $g=t=x+\delta y$ for $t \in E^{(1)}$ and $x, y \in F$. Let $\varrho$ be as defined in Subsection 5.2. Then

$$
\begin{aligned}
& \beta_{\mathcal{V}^{\prime}}^{Y}(g)=\chi((t-1) \delta)(\Delta, \varrho)_{F} \gamma_{F}\left(\Delta, \psi_{0}\right)^{-1}, \\
& \alpha_{\mathcal{V}^{\prime}}(g)= \begin{cases}1, & \text { if } x \neq 1 \bmod \mathfrak{p}_{F} ; \\
\gamma_{F}\left(-\Delta, \psi_{0}\right)(\Delta, 2 y \varrho)_{F}\left(\frac{-1}{\mathbf{f}_{F}}\right)^{\overline{\operatorname{ord}}\left(\psi_{0}\right)}, & \text { if } x \equiv 1 \bmod \mathfrak{p}_{F} .\end{cases}
\end{aligned}
$$


Proof. Like the computation in Subsection 6.1, we have $j(g)=1$. Hence, if $\epsilon=1$,

$$
\begin{aligned}
\beta_{\mathcal{V}^{\prime}}^{Y}(g) & =\chi\left((t-1) r^{-1}\right) \chi(\delta)\left(\Delta, r^{\prime} \delta\right)_{F}(-1,-\Delta)_{F} \gamma_{F}\left(\Delta, \psi_{0}\right)^{-1} \\
& =\chi((t-1) \delta)(\Delta, \varrho)_{F} \gamma_{F}\left(\Delta, \psi_{0}\right)^{-1}
\end{aligned}
$$

If $\epsilon=-1$, we have

$$
\begin{aligned}
\beta_{\mathcal{V}^{\prime}}^{Y}(g) & =\chi\left((t-1) r^{-1}\right)\left(\Delta, r^{\prime}\right)_{F}(-1,-\Delta)_{F} \gamma_{F}\left(\Delta, \psi_{0}\right)^{-1} \\
& =\chi((t-1) \delta)\left(\Delta,(r \delta)^{-1} r^{\prime}\right)_{F}(-1,-\Delta)_{F} \gamma_{F}\left(\Delta, \psi_{0}\right)^{-1} \\
& =\chi((t-1) \delta)(\Delta, \varrho)_{F} \gamma_{F}\left(\Delta, \psi_{0}\right)^{-1} .
\end{aligned}
$$

Suppose that $x \not \equiv 1 \bmod \mathfrak{p}_{F}$. From the result in the proof of Lemma 5.3, we know that $B^{*} / B$ is trivial. Then obviously $m\left(\overline{\iota \mathcal{V}^{\prime}(g)}\right)=1$ and $j\left(\overline{\left(\mathcal{V}^{\prime}(g)\right.}\right)=$ 0 . Thus $\alpha_{\mathcal{V}^{\prime}}(g)=1$ by (6.1.c). If $x \equiv 1 \bmod \mathfrak{p}_{F}$, the lemma follows from the same computation in Subsection 6.1.

7.2. . Keep the assumptions in Subsection 6.2 except that we assume $E$ is a ramified extension here.

Lemma. Suppose that $\mathcal{V}$ is one-dimensional and $\mathcal{V}^{\prime}$ is two-dimensional isotropic. Suppose that $g$ is an element in $U(\mathcal{V})$ and $g \neq 1$. Write $g=t$ for $t \in E^{(1)}$. Then

$$
\begin{aligned}
& \beta_{\mathcal{V}^{\prime}}^{Y}(g)=\chi((t-1) \delta), \\
& \alpha_{\mathcal{V}^{\prime}}(g)=1 .
\end{aligned}
$$

Proof. If $\epsilon=1$, like the computation in Subsection 6.2, we know that $\beta_{\mathcal{V}^{\prime}}^{Y}(g)=\chi(x(g) \delta)=\chi\left(r^{-1}(t-1) \delta\right)=\chi((t-1) \delta)(\Delta, r)_{F}^{2}=\chi((t-1) \delta)$. If $\epsilon=-1$, it is easy to check the above is also true.

We can also check that $B^{*} / B$ is either trivial or four-dimensional. Suppose that $x \not \equiv 1 \bmod \mathfrak{p}_{F}$. If $B^{*}=B$, obviously we have $m\left(\overline{\iota \mathcal{V}^{\prime}(g)}\right)=1$ and $j\left(\overline{\iota_{\mathcal{V}^{\prime}}(g)}\right)=0$. Hence, $\alpha_{\mathcal{V}^{\prime}}(g)=1$. If $B^{*} / B$ is four-dimensional, it is straightforward to check that $j\left(\overline{\left(\mathcal{V}^{\prime}(g)\right.}\right)=2$ and $x\left(\overline{\left(\iota_{\mathcal{V}^{\prime}}(g)\right.}\right)=-\overline{y^{2}(2 \Delta r)^{-2}}$. So from (4.2.a), we see that $m\left(\overline{\iota_{\mathcal{V}^{\prime}}(g)}\right)=\left(\frac{-1}{\mathbf{f}_{F}}\right) \gamma_{\mathbf{f}_{F}}\left(\psi_{0}\right)^{-2}=1$. Similar to the computation in Subsection 6.2, we have $\alpha_{\mathcal{V}^{\prime}}(g)=1$. Next we assume that $x \equiv 1 \bmod \mathfrak{p}_{F}$. Then we know that $\overline{\iota \mathcal{V}^{\prime}(g)}=1$. Hence we have $m\left(\overline{\iota_{\mathcal{V}^{\prime}}(g)}\right)=1$ and $j\left(\overline{\iota \mathcal{V}^{\prime}}(g)\right)=0$. By the exactly same computation in Subsection 6.2, we have $\alpha \mathcal{V}^{\prime}(g)=1$. 
7.3. . In this subsection, we assume that $\mathcal{V}$ is one-dimensional and $\mathcal{V}^{\prime}$ is two-dimensional anisotropic. Let $\mathcal{V}_{i}^{\prime}, r_{i}^{\prime}$ be as defined in Subsection 6.3.

Lemma. Suppose that $\mathcal{V}$ is one-dimensional and $\mathcal{V}^{\prime}$ is two-dimensional anisotropic. Suppose that $g:=t \in E^{(1)}$ is an element in $U(\mathcal{V})$ and $g \neq 1$. Let $\beta_{\mathcal{V}^{\prime}}^{Y}$ be defined with respect to a character $\chi$ of $E^{\times}$such that $\left.\chi\right|_{F^{\times}}=\epsilon_{E / F}^{2}$. Then

$$
\begin{aligned}
& \beta_{\mathcal{V}^{\prime}}^{Y}(g)=-\chi((t-1) \delta), \\
& \alpha \mathcal{V}^{\prime}(g)=\left\{\begin{aligned}
1, & \text { if } x \not \equiv 1 \bmod \mathfrak{p}_{F} ; \\
-1, & \text { if } x \equiv 1 \bmod \mathfrak{p}_{F} .
\end{aligned}\right.
\end{aligned}
$$

Proof. If $\epsilon=-1$, then $\beta_{\mathcal{V}^{\prime}}^{Y}(g)=\chi(x(g))\left(\Delta, r_{1}^{\prime} r_{2}^{\prime}\right)_{F}(\Delta, \Delta)_{F}=-\chi((t-1) \delta)$. If $\epsilon=-1$, this is true again as in Subsection 6.3. By the same argument in Subsection 6.3, we see that $\alpha_{\mathcal{V}^{\prime}}(g)=1$ if $x \not \equiv 1 \bmod \mathfrak{p}_{F}$ and $\alpha_{\mathcal{V}^{\prime}}(g)=-1$ if $x \equiv 1 \bmod \mathfrak{p}_{F}$.

7.4. . Let $r^{\prime}$ be an element in $F^{\times}$such that $r^{\prime}:=\mathbf{h}^{\prime}\left(v^{\prime}, v^{\prime}\right)$ if $\epsilon=-1$; $r^{\prime} \delta:=\mathbf{h}^{\prime}\left(v^{\prime}, v^{\prime}\right)$ if $\epsilon=1$ for some nonzero $v^{\prime} \in \mathcal{V}^{\prime}$.

Lemma. Suppose that $\mathcal{V}$ is two-dimensional isotropic and $\mathcal{V}^{\prime}$ is one-dimensional. Let $g_{1}:=\left[\begin{array}{cc}\tau(k) & 0 \\ 0 & k^{-1}\end{array}\right], g_{2}:=\left[\begin{array}{cc}0 & \epsilon k^{-1} \\ \tau(k) & 0\end{array}\right]$ for some $k \in E^{\times}$such that $g_{1}, g_{2}$ are in $U(\mathcal{V})_{L}$ and $g_{1} \neq 1$. Write $k=x_{0}+\delta y_{0}$ for $x_{0}, y_{0} \in F$. Then

$$
\begin{aligned}
& \beta_{\mathcal{V}^{\prime}}^{Y}(g)= \begin{cases}\chi(\tau(k)), & \text { if } g=g_{1} ; \\
\chi(\tau(k))\left(\Delta,-r^{\prime}\right)_{F} \gamma_{\mathbf{f}_{F}}\left(\bar{\psi}_{0}\right)^{-1}\left(\frac{-1}{\mathbf{f}_{F}}\right)^{\overline{\operatorname{ord}}\left(\psi_{0}\right)}, & \text { if } g=g_{2} \text { and } \epsilon=-1 ; \\
\chi(\tau(k) \delta)\left(\Delta, r^{\prime}\right)_{F} \gamma_{\mathbf{f}_{F}}\left(\bar{\psi}_{0}\right)^{-1}\left(\frac{-1}{\mathbf{f}_{F}}\right)^{\overline{\operatorname{ord}}\left(\psi_{0}\right)}, & \text { if } g=g_{2} \text { and } \epsilon=1,\end{cases} \\
& \alpha_{\mathcal{V}^{\prime}}(g)= \begin{cases}1, & \text { if } g=g_{1} \text { and } B^{*} / B \text { is trivial; } \\
\left(\frac{\bar{x}_{0}}{\mathbf{f}_{F}}\right), & \text { if } g=g_{1} \text { and } B^{*} / B \text { is not trivial; } \\
1, & \text { if } g=g_{2} \text { and } B^{*} / B \text { is trivial; } \\
\left(\frac{-\overline{2 x_{0} r^{\prime}}}{\mathbf{f}_{F}}\right) \gamma_{\mathbf{f}_{F}}\left(\bar{\psi}_{0}\right), & \text { if } g=g_{2} \text { and } B^{*} / B \text { is not trivial. }\end{cases}
\end{aligned}
$$

Proof. First, suppose that $g=g_{1}$. We have $j(g)=0$. Hence, $\beta_{\mathcal{V}^{\prime}}^{Y}(g)=$ $\chi(\tau(k))$. If $B^{*} / B$ is trivial, it is clear that $m\left(\overline{\iota \mathcal{V}^{\prime}(g)}\right)=1$ and $j\left(\overline{\iota_{\mathcal{V}^{\prime}}(g)}\right)=0$. Therefore, we have $\alpha_{\mathcal{V}^{\prime}}(g)=1$. If $B^{*} / B$ is not trivial, then it is twodimensional. And we have $j\left(\overline{\iota \mathcal{V}^{\prime}}(g)\right)=0, m\left(\overline{\iota_{\mathcal{V}^{\prime}}(g)}\right)=\gamma_{\mathbf{f}_{F}}\left(\bar{x}_{0}, \bar{\psi}_{0}\right)^{-1}=\left(\frac{\bar{x}_{0}}{\mathbf{f}_{F}}\right)$. Hence, $\alpha_{\mathcal{V}^{\prime}}(g)=\left(\frac{\bar{x}_{0}}{\mathbf{f}_{F}}\right)$. 
Next, suppose that $g=g_{2}$ and $\epsilon=-1$. Then $j(g)=1$. Hence, from $[\mathbf{K l}]$ Theorem 3.1, we have

$$
\begin{aligned}
\beta_{\mathcal{V}^{\prime}}^{Y}(g) & =\chi(\tau(k))\left(\Delta, r^{\prime}\right)_{F} \gamma_{F}\left(-\Delta, \psi_{0}\right)^{-1} \gamma_{F}\left(-1, \psi_{0}\right) \\
& =\chi(\tau(k))\left(\Delta, r^{\prime}\right)_{F} \gamma_{F}\left(\Delta, \psi_{0}\right)^{-1}(\Delta,-1)_{F} \\
& =\chi(\tau(k))\left(\Delta,-r^{\prime}\right)_{F} \gamma_{\mathbf{f}_{F}}\left(\bar{\psi}_{0}\right)^{-1}\left(\frac{-1}{\mathbf{f}_{F}}\right)^{\overline{\operatorname{ord}}\left(\psi_{0}\right)} .
\end{aligned}
$$

If $\epsilon=1$, then

$$
\begin{aligned}
\beta_{\mathcal{V}^{\prime}}^{Y}(g) & =\chi(\tau(k) \delta)\left(\Delta, r^{\prime} \Delta\right)_{F} \gamma_{F}\left(-\Delta, \psi_{0}\right)^{-1} \gamma_{F}\left(-1, \psi_{0}\right) \\
& =\chi(\tau(k) \delta)\left(\Delta, r^{\prime}\right)_{F} \gamma_{\mathbf{f}_{F}}\left(\bar{\psi}_{0}\right)^{-1}\left(\frac{-1}{\mathbf{f}_{F}}\right)^{\overline{\operatorname{ord}}\left(\psi_{0}\right)} .
\end{aligned}
$$

Let $\left\{w_{1}, \ldots, w_{4}\right\}$ be the basis of $\mathcal{W}$ given in Subsection 6.4. Then

$$
\iota^{\prime}(g)=\left[\begin{array}{cccc}
0 & 0 & \frac{-x_{0} r^{\prime} \Delta}{k \tau(k)} & \frac{y_{0} r^{\prime} \Delta}{k \tau(k)} \\
0 & 0 & \frac{-y_{0} r^{\prime} \Delta}{k \tau(k)} & \frac{x_{0} r^{\prime}}{k \tau(k)} \\
\frac{-x_{0}}{r^{\prime} \Delta} & \frac{-y_{0}}{r^{\prime}} & 0 & 0 \\
\frac{y_{0}}{r^{\prime}} & \frac{x_{0}}{r^{\prime}} & 0 & 0
\end{array}\right]
$$

with respect to the basis. If $B^{*} / B$ is trivial, then $m\left(\overline{\left(\mathcal{V}^{\prime}(g)\right.}\right)=1$ and $j\left(\overline{\iota \mathcal{V}^{\prime}(g)}\right)=0$. Therefore, we have $\alpha_{\mathcal{V}^{\prime}}(g)=1$. If $B^{*} / B$ is not trivial, then it is two-dimensional. And we have $j\left(\overline{\iota \mathcal{V}^{\prime}(g)}\right)=1$ and $x\left(\overline{\left(\mathcal{V}^{\prime}(g)\right.}\right)=-\overline{x_{0} / r^{\prime} \Delta}$. Hence $\alpha \mathcal{V}^{\prime}(g)=\left(\frac{-\overline{2 x_{0} r^{\prime}}}{\mathbf{f}_{F}}\right) \gamma_{\mathbf{f}_{F}}\left(\bar{\psi}_{0}\right)$.

7.5. . Keep the assumptions in Subsection 6.4 except that we assume $E$ is a ramified extension here.

Lemma. Suppose that both $\mathcal{V}$ and $\mathcal{V}^{\prime}$ are two-dimensional and isotropic. Let $g_{1}:=\left[\begin{array}{cc}\tau(k) & 0 \\ 0 & k^{-1}\end{array}\right], g_{2}:=\left[\begin{array}{cc}0 & \epsilon k^{-1} \\ \tau(k) & 0\end{array}\right]$ for some $k \in E^{\times}$such that $g_{1}, g_{2}$ are in $U(\mathcal{V})_{L}$ and $g_{1} \neq 1$. Write $k=x_{0}+\delta y_{0}$ for $x_{0}, y_{0} \in F$. Then

$$
\begin{aligned}
& \beta_{\mathcal{V}^{\prime}}^{Y}(g)= \begin{cases}\chi(\tau(k)), & \text { if } g=g_{1} ; \\
\chi(\tau(k)), & \text { if } g=g_{2} \text { and } \epsilon=-1 ; \\
\chi(\tau(k) \delta), & \text { if } g=g_{2} \text { and } \epsilon=1,\end{cases} \\
& \alpha_{\mathcal{V}^{\prime}}(g)=1 .
\end{aligned}
$$

Proof. Suppose that $g=g_{1}$. Then $j(g)=0$ and $\beta_{\mathcal{V}^{\prime}}^{Y}(g)=\chi(\tau(k))$. The proof for $\alpha_{\mathcal{V}^{\prime}}(g)=1$ is exactly the same as in Subsection 6.5.

Next suppose that $g=g_{2}$ and $\epsilon=-1$. Then

$$
\begin{aligned}
\beta_{\mathcal{V}^{\prime}}^{Y}(g) & =\chi(\tau(k))(\Delta,-1)_{F} \gamma_{F}\left(-\Delta, \psi_{0}\right)^{-2} \gamma_{F}\left(-1, \psi_{0}\right)^{2} \\
& =\chi(\tau(k))(\Delta,-1)_{F}(\Delta,-1)_{F}=\chi(\tau(k)) .
\end{aligned}
$$


If $\epsilon=1$, the computation is similar. If $B^{*} / B$ is trivial, then $m\left(\overline{\left(\mathcal{V}^{\prime}(g)\right.}\right)=1$ and $j\left(\overline{\iota^{\prime}(g)}\right)=0$. Therefore we have $\alpha_{\mathcal{V}^{\prime}}(g)=1$. If $B^{*} / B$ is not trivial, then it is four-dimensional; and we have $j\left(\overline{\iota_{\mathcal{V}^{\prime}}(g)}\right)=2$ and $x\left(\overline{\iota_{\mathcal{V}}(g)}\right)=\left(\frac{-1}{\mathbf{f}_{F}}\right)$. Hence, $\alpha_{\mathcal{V}^{\prime}}(g)=\left(\frac{-1}{\mathbf{f}_{F}}\right) \gamma_{\mathbf{f}_{F}}\left(\bar{\psi}_{0}\right)^{2}=1$.

7.6. . In this subsection, we assume that $\mathcal{V}$ is two-dimensional isotropic and $\mathcal{V}^{\prime}$ is two-dimensional anisotropic. Let $\mathcal{V}_{i}^{\prime}, r_{i}^{\prime}$ be as defined in Subsection 6.3.

Lemma. Suppose that $\mathcal{V}$ is two-dimensional isotropic and $\mathcal{V}^{\prime}$ is two-dimensional anisotropic. Let $\beta_{\mathcal{V}^{\prime}}^{Y}$ be defined with respect to $\chi$. Let $g_{1}:=\left[\begin{array}{cc}\tau(k) & 0 \\ 0 & k^{-1}\end{array}\right]$, $g_{2}:=\left[\begin{array}{cc}0 & \epsilon k^{-1} \\ \tau(k) & 0\end{array}\right]$ for some $k \in E^{\times}$such that $g_{1}, g_{2}$ are in $U(\mathcal{V})_{L}$ and $g_{1} \neq 1$. Write $k=x_{0}+\delta y_{0}$ for $x_{0}, y_{0} \in F$. Then

$$
\begin{gathered}
\beta_{\mathcal{V}^{\prime}}^{Y}(g)= \begin{cases}\chi(\tau(k)), & \text { if } g=g_{1} ; \\
-\chi(\tau(k)), & \text { if } g=g_{2} \text { and } \epsilon=-1 ; \\
-\chi(\tau(k) \delta), & \text { if } g=g_{2} \text { and } \epsilon=1,\end{cases} \\
\alpha_{\mathcal{V}^{\prime}}(g)=\left\{\begin{array}{cc}
1, & \text { if } g=g_{1} \text { is trivial; } \\
1, & \text { if } g=g_{2} \text { and } B^{*} / B \text { is trivial; } \\
-1, & \text { if } g=g_{2} \text { and } B^{*} / B \text { is not trivial. }
\end{array}\right.
\end{gathered}
$$

Proof. The proof for $\beta_{\mathcal{V}^{\prime}}^{Y}(g)$ is analogous to case in Lemma 7.3.

Next we compute $\alpha_{\mathcal{V}^{\prime}}(g)$. Let $B_{1}, B_{2}$ be as defined in the proof of Lemma 6.6. Now we notice that either both $B_{1}^{*} / B_{1}, B_{2}^{*} / B_{2}$ are trivial or both are two-dimensional. Hence, it is clear that $\alpha_{\mathcal{V}^{\prime}}(g)=\alpha_{\mathcal{V}_{1}^{\prime}}(g) \alpha_{\mathcal{V}_{2}^{\prime}}(g)=1$ if $g=g_{1}$. So now suppose that $g=g_{2}$. If $B^{*} / B=B_{1}^{*} / B_{1} \oplus B_{2}^{*} / B_{2}$ is trivial, it is clear that $\alpha_{\mathcal{V}^{\prime}}(g)=1$ from Lemma 7.4. If $B^{*} / B$ is four-dimensional, then $\alpha_{\mathcal{V}^{\prime}}(g)=-\left(\frac{-1}{\mathbf{f}_{F}}\right) \gamma_{\mathbf{f}_{F}}\left(\bar{\psi}_{0}\right)^{2}=-1$ from Lemma 7.4.

7.7. . In this subsection, we assume $\mathcal{V}$ two-dimensional isotropic, $\mathcal{V}^{\prime}$ onedimensional and $q=3$. We shall consider the case that $\mathbf{l}$ is trivial and $\mathbf{l}^{*}$ is two-dimensional symplectic space. Fix a standard basis $\left\{v_{1}, v_{2}\right\}$ of $\mathcal{V}$. Choose a nonzero element $v^{\prime}$ in $\mathcal{V}^{\prime}$ such that $\operatorname{ord}(\varrho)=0$ or 1 where $\varrho:=\mathbf{h}^{\prime}\left(v^{\prime}, v^{\prime}\right)$. Let $Y^{\circ}:=E v_{2}$ and $Y:=Y^{\circ} \otimes \mathcal{V}^{\prime}$.

Lemma. Suppose that $\mathcal{V}$ is two-dimensional isotropic and $\mathcal{V}^{\prime}$ is one-dimensional. Suppose that $g:=\left[\begin{array}{ll}1 & a \\ 0 & 1\end{array}\right] \in U(\mathcal{V})$ for some a such that $a=\epsilon \tau(a)$ and $g \in U(\mathcal{V})_{L}$. Then

$$
\begin{aligned}
& \beta_{\mathcal{V}^{\prime}}^{Y}(g)=1 \\
& \alpha_{\mathcal{V}^{\prime}}(g)=1
\end{aligned}
$$


Proof. It is clear that $x(g)=1$ and $j(g)=0$. By [Kl] Theorem 3.1, we see that $\beta_{\mathcal{V}^{\prime}}^{Y}(g)=\chi(x(g))=\chi(1)=1$.

Let $w_{1}:=\frac{1}{\varrho} v_{1} \otimes v^{\prime}, w_{2}:=\frac{-\delta}{\Delta \varrho} v_{1} \otimes v^{\prime}, w_{3}:=v_{2} \otimes v^{\prime}$, and $w_{4}:=\delta v_{2} \otimes v^{\prime}$. The the set $\left\{w_{1}, w_{2}, w_{3}, w_{4}\right\}$ is a standard basis of $\mathcal{W}:=\mathcal{V} \otimes \mathcal{V}^{\prime}$. Then $Y=F w_{3}+F w_{4}$. We have

$$
\iota \mathcal{V}^{\prime}(g)=\left[\begin{array}{cccc}
1 & 0 & \varrho a & 0 \\
0 & 1 & 0 & -\Delta \varrho a \\
0 & 0 & 1 & 0 \\
0 & 0 & 0 & 1
\end{array}\right] \in \operatorname{Sp}(\mathcal{W})
$$

with respect to the basis $\left\{w_{1}, w_{2}\right\}$ of $\mathcal{W}$. It is clear that $B^{*} / B$ is twodimensional, $j\left(\overline{\left(\mathcal{V}^{\prime}(g)\right.}\right)=0$ and $x\left(\overline{\iota_{\mathcal{V}^{\prime}}(g)}\right)=1$. Hence $\alpha_{\mathcal{V}^{\prime}}(g)=1$.

7.8. . In this subsection, we assume that both $\mathcal{V}$ and $\mathcal{V}^{\prime}$ two-dimensional isotropic, and $q=3$. We shall consider the case that $\mathbf{l}$ is trivial and $\mathbf{l}^{*}$ is twodimensional symplectic space. Fix a standard basis $\left\{v_{1}, v_{2}\right\}$ (resp. $\left\{v_{1}^{\prime}, v_{2}^{\prime}\right\}$ ) of $\mathcal{V}\left(\right.$ resp. $\left.\mathcal{V}^{\prime}\right)$. Let $Y^{\circ}:=E v_{2}$ and $Y:=Y^{\circ} \otimes \mathcal{V}^{\prime}$.

Lemma. Suppose that both $\mathcal{V}$ and $\mathcal{V}^{\prime}$ are two-dimensional isotropic. Let $g:=\left[\begin{array}{ll}1 & a \\ 0 & 1\end{array}\right] \in U(\mathcal{V})$ for some a such that $a=\epsilon \tau(a)$ and $g \in U(\mathcal{V})_{L}$. Then

$$
\begin{aligned}
& \beta_{\mathcal{V}^{\prime}}^{Y}(g)=1, \\
& \alpha_{\mathcal{V}^{\prime}}(g)=1 .
\end{aligned}
$$

Proof. As in Subsection 7.7, we have $x(g)=1$ and $j(g)=0$. Then clearly $\beta_{\mathcal{V}^{\prime}}^{Y}(g)=\chi(x(g))=\chi(1)=1$.

Assume that $\epsilon=1$. Let $w_{1}:=v_{1} \otimes v_{1}^{\prime}, w_{2}=-\frac{\delta}{\Delta} v_{1} \otimes v_{1}^{\prime}, w_{3}:=v_{1} \otimes v_{2}^{\prime}$, $w_{4}:=-\frac{\delta}{\Delta} v_{1} \otimes v_{2}^{\prime} . \quad w_{5}:=v_{2} \otimes v_{2}^{\prime}, w_{6}:=\delta v_{2} \otimes v_{2}^{\prime}, w_{7}:=v_{2} \otimes v_{1}^{\prime}$, and $w_{8}:=\delta v_{2} \otimes v_{1}^{\prime}$. The the set $\left\{w_{1}, \ldots, w_{8}\right\}$ is a standard basis of $\mathcal{W}:=\mathcal{V} \otimes \mathcal{V}^{\prime}$. Then $Y$ is the $F$-space spanned by $\left\{w_{5}, \ldots, w_{8}\right\}$. We have

$$
\iota_{\mathcal{V}^{\prime}}(g)=\left[\begin{array}{cccccccc}
1 & 0 & 0 & 0 & 0 & 0 & 0 & \frac{a}{\delta} \\
0 & 1 & 0 & 0 & 0 & 0 & -\delta a & 0 \\
0 & 0 & 1 & 0 & 0 & \frac{a}{\delta} & 0 & 0 \\
0 & 0 & 0 & 1 & -\delta a & 0 & 0 & 0 \\
0 & 0 & 0 & 0 & 1 & 0 & 0 & 0 \\
0 & 0 & 0 & 0 & 0 & 1 & 0 & 0 \\
0 & 0 & 0 & 0 & 0 & 0 & 1 & 0 \\
0 & 0 & 0 & 0 & 0 & 0 & 0 & 1
\end{array}\right]
$$

with respect to the basis $\left\{w_{1}, w_{2}, w_{3}, w_{4}\right\}$ of $\mathcal{W}$. It is clear to see that the space $B^{*} / B$ is either trivial or four dimensional. If $B^{*} / B$ is trivial, then $m\left(\overline{\iota \mathcal{V}^{\prime}}(g)\right)=1$ and $j\left(\overline{\left(\mathcal{V}^{\prime}\right.}(g)\right)=0$. Therefore, $\alpha_{\mathcal{V}^{\prime}}(g)=1$ by (7.8.a). If 
$B^{*} / B$ is four-dimensional, then it is clear from (7.8.a) that $j\left(\overline{\iota \mathcal{V}^{\prime}(g)}\right)=0$ and $m\left(\overline{\iota^{\prime}(g)}\right)=1$. Therefore, $\alpha_{\mathcal{V}^{\prime}}(g)=1$. If $\epsilon=-1$, the computation is similar.

\subsection{Proof of Proposition 3.3 for $\mathcal{V} \epsilon$-hermitian and $E$ ramified:} Part (I). Since now $E$ is a ramified quadratic extension of $F$, we know that $\mathbf{f}_{E}=\mathbf{f}_{F}$, one of $\mathbf{l}, \mathbf{l}^{*}$ is a quadratic space and the other is a symplectic space. Without loss of generality, we assume that $\mathbf{l}$ is a quadratic space and $\mathbf{l}^{*}$ is a symplectic space. In this subsection, we assume that $q>3$. As in Subsection 6.7, we have an $L^{\prime}$-admissible decomposition $\mathcal{V}^{\prime}=\bigoplus_{i} \mathcal{V}_{i}^{\prime}$ of nondegenerate subspaces such that each $\mathcal{V}_{i}^{\prime}$ is either one-dimensional or a hyperbolic plane, and we have $\alpha_{\mathcal{V}^{\prime}}(g) \beta_{\mathcal{V}^{\prime}}^{Y}(g)=\prod_{i} \alpha_{\mathcal{V}_{i}^{\prime}}(g) \beta_{\mathcal{V}_{i}^{\prime}}^{Y_{i}}(g)$ under a proper choice of $\chi_{i}, \chi$ where $Y_{i}:=Y^{\circ} \otimes \mathcal{V}_{i}^{\prime}$. We also have an $L$-admissible decomposition $\mathcal{V}=\mathcal{V}_{1} \oplus \mathcal{V}_{2}$ such that $L \cap \mathcal{V}_{1}=L^{*} \cap \mathcal{V}_{1}$ and $L \cap \mathcal{V}_{2}=\varpi_{E}\left(L^{*} \cap\right.$ $\left.\mathcal{V}_{2}\right)$. It is obvious that $\operatorname{dim}(\mathbf{l})=\operatorname{dim}\left(\mathcal{V}_{1}\right)$ and $\operatorname{dim}\left(\mathbf{l}^{*}\right)=\operatorname{dim}\left(\mathcal{V}_{2}\right)$. Now we have the following cases. From our assumption that $\mathbf{l}^{*}$ is a symplectic space, we see that $\mathcal{V}_{2}$ must be even-dimensional. From Subsection 1.4, we know that $S U(\mathcal{V})_{L} /\left[U(\mathcal{V})_{L}, U(\mathcal{V})_{L}\right]$ is nontrivial if and only if the dimension of $\mathcal{V}_{1}$ is great than one. (Note that we assume that $q>3$.) So we assume that the dimension of $\mathcal{V}_{1}$ is greater than 1.

Suppose that $\mathcal{V}_{1}$ is isotropic. Choose $L$-admissible hyperbolic planes $\mathcal{V}_{1}^{0}$ in $\mathcal{V}_{1}$. Let $g:=\left[\begin{array}{cc}\tau(k) & 0 \\ 0 & k^{-1}\end{array}\right] \in U\left(\mathcal{V}_{1}^{0}\right) \subseteq U\left(\mathcal{V}_{1}\right) \subseteq U(\mathcal{V})$ for some $k \in \mathcal{O}_{F}^{\times}-\mathfrak{p}_{F}^{\times}$ such that $k$ is not a square. Hence $g$ is a representative of the nontrivial $\left[U(\mathcal{V})_{L}, U(\mathcal{V})_{L}\right]$-coset in $S U(\mathcal{V})_{L}$. Consider the dual pairs $\left(U\left(\mathcal{V}_{1}^{0}\right), U\left(\mathcal{V}_{i}^{\prime}\right)\right)$. We have

$$
\chi_{i}(\tau(k))=\chi_{i}(k)=(\Delta, k)_{F}^{m_{i}}=\left\{\begin{aligned}
-1, & \text { if } m_{i}=1 ; \\
1, & \text { if } m_{i}=2
\end{aligned}\right.
$$

where $m_{i}$ is the dimension of $\mathcal{V}_{i}^{\prime}$. Let $L_{0}:=L \cap \mathcal{V}_{1}^{0}, L_{i}^{\prime}:=L^{\prime} \cap \mathcal{V}_{i}$ and $B_{i}=B\left(L_{0}, L_{i}^{\prime}\right)$. From the assumption, we know that $L_{0}^{*} / L_{0}$ is trivial and $L_{0} / \varpi_{E} L_{0}^{*}$ is a two-dimensional quadratic space. If $\mathcal{V}_{i}^{\prime}$ is one-dimensional, then the space $L_{i}^{\prime *} / L_{i}^{\prime} \oplus L_{i}^{\prime} / \varpi_{E} L_{i}^{\prime *}$ must be a one-dimensional quadratic space. Hence the quotient $B_{i}^{*} / B_{i}$ must be trivial. Then from Lemmas 7.4 and 7.5 , we see that

$$
\alpha_{\mathcal{V}_{i}^{\prime}}(g) \beta_{\mathcal{V}_{i}^{\prime}}^{Y_{i}}(g)=\left\{\begin{aligned}
-1, & \text { if } \mathcal{V}_{i}^{\prime} \text { is one-dimensional } \\
1, & \text { if } \mathcal{V}_{i}^{\prime} \text { is two-dimensional isotropic. }
\end{aligned}\right.
$$

If the dimension of $\mathcal{V}^{\prime}$ is odd, there is exactly one odd-dimensional $\mathcal{V}_{i}^{\prime}$. If the dimension of $\mathcal{V}^{\prime}$ is even, there are either two or none odd-dimensional 
$\mathcal{V}_{i}^{\prime}$. Therefore, we conclude that

$$
\alpha_{\mathcal{V}^{\prime}}(g) \beta_{\mathcal{V}^{\prime}}^{Y}(g)=\left\{\begin{aligned}
-1, & \text { if } \mathcal{V}^{\prime} \text { is odd-dimensional } \\
1, & \text { if } \mathcal{V}^{\prime} \text { is even-dimensional }
\end{aligned}\right.
$$

Next, suppose that $\mathcal{V}_{1}$ is anisotropic. Hence $\mathcal{V}_{1}$ is two-dimensional in our situation. As in (iv) of Subsection 1.4, we have two $L$-admissible subspaces $E v_{1}, E v_{2}$ such that $\left(\Delta, \mathbf{h}\left(v_{1}, v_{1}\right) \mathbf{h}\left(v_{2}, v_{2}\right)\right)_{F}=-1$. Let $g_{i}$ be the element in $U(\mathcal{V})$ such that $\left.g_{i}\right|_{E v_{i}}=-1$ and $\left.g_{i}\right|_{\left(E v_{i}\right)^{\perp}}=1$. Then it is clear that $g_{1} g_{2} \in S U(\mathcal{V})_{L}$ and the image of $g_{1} g_{2}$ in $S U(\mathcal{V})_{L} /\left[U(\mathcal{V})_{L}, U(\mathcal{V})_{L}\right]$ generates $S U(\mathbf{l}) /[U(\mathbf{l}), U(\mathbf{l})]$, which is isomorphic to $\mathbf{Z} / 2 \mathbf{Z}$. So we need to compute $\alpha \mathcal{V}^{\prime}\left(g_{1} g_{2}\right) \beta_{\mathcal{V}^{\prime}}^{Y}\left(g_{1} g_{2}\right)$. Since $\alpha \mathcal{V}^{\prime} \beta_{\mathcal{V}^{\prime}}^{Y}$ is a character of $U(\mathcal{V})_{L}$, we have

$$
\alpha_{\mathcal{V}^{\prime}}\left(g_{1} g_{2}\right) \beta_{\mathcal{V}^{\prime}}^{Y}\left(g_{1} g_{2}\right)=\alpha_{\mathcal{V}^{\prime}}\left(g_{1}\right) \alpha_{\mathcal{V}^{\prime}}\left(g_{2}\right) \beta_{\mathcal{V}^{\prime}}^{Y}\left(g_{1}\right) \beta_{\mathcal{V}^{\prime}}^{Y}\left(g_{2}\right) .
$$

Let $\mathcal{V}^{\prime}=\bigoplus_{i} \mathcal{V}_{i}^{\prime}$ be the decomposition in the previous paragraph. If $\mathcal{V}_{i}^{\prime}$ is one-dimensional, let $\varrho_{j, i}$ denote the number $\varrho$ in Subsection 7.1 for the pair $\left(U\left(E v_{j}\right), U\left(\mathcal{V}_{i}^{\prime}\right)\right)$. From the computation in Subsection 7.1, we know that

$$
\alpha_{\mathcal{V}_{i}^{\prime}}\left(g_{1}\right) \alpha_{\mathcal{V}_{i}^{\prime}}\left(g_{2}\right) \beta_{\mathcal{V}_{i}^{\prime}}^{Y_{i}}\left(g_{1}\right) \beta_{\mathcal{V}_{i}^{\prime}}^{Y_{i}}\left(g_{2}\right)=\chi_{i}\left((-2)^{2} \delta^{2}\right)\left(\Delta, \varrho_{1, i} \varrho_{2, i}\right)_{F} \gamma_{F}\left(\Delta, \psi_{0}\right)^{-2} .
$$

From the assumption, we have $\left(\Delta, \varrho_{1, i} \varrho_{2, i}\right)_{F}=-1$. Therefore,

$$
\alpha_{\mathcal{V}_{i}^{\prime}} \beta_{\mathcal{V}_{i}^{\prime}}^{Y_{i}}(g)=-(\Delta, \Delta)_{F} \gamma_{F}\left(\Delta, \psi_{0}\right)^{-2}=-1
$$

If $\mathcal{V}_{i}^{\prime}$ is a hyperbolic plane, from the computation in Subsection 7.2, we know that

$$
\begin{aligned}
\alpha_{\mathcal{V}_{i}^{\prime}}\left(g_{1}\right) \beta_{\mathcal{V}_{i}^{\prime}}^{Y_{i}}\left(g_{1}\right) \alpha_{\mathcal{V}_{i}^{\prime}}\left(g_{2}\right) \beta_{\mathcal{V}_{i}^{\prime}}^{Y_{i}}\left(g_{2}\right) & =\chi_{i}\left(x\left(g_{1}\right) x\left(g_{2}\right)\right) \chi(\delta)^{2} \\
& =\chi_{i}\left((-2)^{2}\right)\left((\Delta, \Delta)_{F}\right)^{2}=1 .
\end{aligned}
$$

If the dimension of $\mathcal{V}^{\prime}$ is odd, there is exactly one odd-dimensional $\mathcal{V}_{i}^{\prime}$. If the dimension of $\mathcal{V}^{\prime}$ is even, there are either two or none odd-dimensional $\mathcal{V}_{i}^{\prime}$. Therefore, we conclude that

$$
\alpha \mathcal{V}^{\prime}(g) \beta_{\mathcal{V}^{\prime}}^{Y}(g)=\left\{\begin{aligned}
-1, & \text { if the dimensional of } \mathcal{V}^{\prime} \text { is odd } \\
1, & \text { if the dimensional of } \mathcal{V}^{\prime} \text { is even }
\end{aligned}\right.
$$

Combining (7.9.a) and (7.9.b), Proposition 3.3 is proved when $\mathcal{V}$ is an $\epsilon$-hermitian space over a ramified extension and $q>3$.

7.10. Proof of Proposition 3.3 for $\mathcal{V} \epsilon$-hermitian and $E$ ramified: Part (II). In this subsection, we assume that $q=3$. Let $\mathcal{V}=\mathcal{V}_{1} \oplus \mathcal{V}_{2}$ be the $L$-admissible decomposition as in Subsection 7.9. In addition to the result proved in Subsection 7.9, we also need to consider the case for $\mathcal{V}_{2}$ two-dimensional. Fix a standard basis $\left\{v_{3}, v_{4}\right\}$ of $\mathcal{V}_{2}$. We have to compute 
$\alpha \mathcal{V}^{\prime}(g) \beta_{\mathcal{V}^{\prime}}^{Y}(g)$ for $g:=\left[\begin{array}{ll}1 & a \\ 0 & 1\end{array}\right]$ as in Subsection 7.7 or 7.8 . Let $\mathcal{V}^{\prime}=\bigoplus_{i} \mathcal{V}_{i}^{\prime}$ be the $L^{\prime}$-admissible decomposition as in Subsection 7.9. From Lemmas 7.7 and 7.8, we see that $\alpha_{\mathcal{V}_{i}^{\prime}}(g) \beta_{\mathcal{V}_{i}^{\prime}}^{Y_{i}}(g)=1$ for each $i$. Therefore,

$$
\alpha_{\mathcal{V}^{\prime}}(g) \beta_{\mathcal{V}^{\prime}}^{Y}(g)=\prod_{i} \alpha_{\mathcal{V}_{i}^{\prime}}(g) \beta_{\mathcal{V}_{i}^{\prime}}^{Y_{i}}(g)=1
$$

with respect to the characters $\chi_{i}, \chi$ defined in Subsection 3.6. Combining the result of Subsection 7.9, we have proved Proposition 3.3 when $\mathcal{V}$ is an $\epsilon$-hermitian space over a ramified quadratic extension of $F$ and $q=3$.

\section{Proof of Proposition 3.3 for orthogonal groups.}

Keep the notation in Subsection 3.3. In this section, we assume that $\mathcal{V}$ is a quadratic space. The computation in this section is similar to but easier than that in Sections 6 and 7. As usual, fix a nontrivial character $\psi$ of $F$.

8.1. . In this subsection, we assume that $\mathcal{V}$ is one-dimensional and $\mathcal{V}^{\prime}$ is twodimensional (isotropic). Now $\mathcal{V}$ is anisotropic, so we embed $\mathcal{V}$ into $\mathcal{V}+\overline{\mathcal{V}}$, which is a direct sum of hyperbolic planes as described in Subsection 3.2.

Lemma. Suppose that $\mathcal{V}$ is one-dimensional and $\mathcal{V}^{\prime}$ is two-dimensional. Let $g:=-1 \in U(\mathcal{V})$. Then

$$
\begin{aligned}
& \beta_{\mathcal{V}^{\prime}}^{Y}(g)=1, \\
& \alpha_{\mathcal{V}^{\prime}}(g)=1 .
\end{aligned}
$$

Proof. From $[\mathbf{K l}]$ Theorem 3.1, we know that $\beta_{\mathcal{V}^{\prime}}^{Y}(g)=1$.

Next, we compute $\alpha_{\mathcal{V}^{\prime}}(g)$. Let $v$ be a nonzero element in $\mathcal{V}$ such that $\operatorname{ord}(\varrho)=0$ or 1 where $\varrho:=\mathbf{h}(v, v)$. Let $\left\{v_{1}^{\prime}, v_{2}^{\prime}\right\}$ be a standard basis of $\mathcal{V}^{\prime}$. Let $w_{1}:=\frac{1}{2 \varrho}(v-\widehat{v}) \otimes v_{1}^{\prime}, w_{2}:=\frac{1}{2 \varrho}(v-\widehat{v}) \otimes v_{2}^{\prime}, w_{3}:=(v+\widehat{v}) \otimes v_{2}^{\prime}$, $w_{4}:=(v+\widehat{v}) \otimes v_{1}^{\prime}$. The set $\left\{w_{1}, w_{2}, w_{3}, w_{4}\right\}$ is a standard basis of $\mathcal{W}+\overline{\mathcal{W}}$. Then,

$$
\iota \mathcal{V}^{\prime}(g)=\left[\begin{array}{cccc}
0 & 0 & 0 & -2 \varrho \\
0 & 0 & -2 \varrho & 0 \\
0 & \frac{-1}{2 \varrho} & 0 & 0 \\
\frac{-1}{2 \varrho} & 0 & 0 & 0
\end{array}\right] \in \operatorname{Sp}(\mathcal{W}+\overline{\mathcal{W}})
$$

with respect to the basis $\left\{w_{1}, w_{2}, w_{3}, w_{4}\right\}$. We know that $X=F w_{1}+F w_{2}$ and $Y=F w_{3}+F w_{4}$. Now $B:=B\left(L+\bar{L}, L^{\prime}\right)$ where $\bar{L}$ be the good lattice in $\overline{\mathcal{V}}$ corresponding to $L$. It is clear that either $B^{*} / B$ is trivial or four-dimensional. Suppose that $B^{*} / B$ is trivial. Then $A=B$ and $\iota_{\mathcal{V}^{\prime}}(g)$ stabilizes $A, m\left(\overline{\iota_{\mathcal{V}^{\prime}}(g)}\right)=1$ and $j\left(\overline{\iota_{\mathcal{V}^{\prime}}(g)}\right)=0$. It is straightforward to check that (6.1.c) is true in this case. Hence $\alpha_{\mathcal{V}^{\prime}}(g)=1$. Next, suppose 
that $B^{*} / B$ is four-dimensional. Clearly $j\left(\overline{\left(\mathcal{V}^{\prime}(g)\right.}\right)=2$ from (8.1.a) and $m\left(\overline{\iota \mathcal{V}^{\prime}}(g)\right)=\left(\frac{x\left(\overline{\iota_{\mathcal{V}^{\prime}}(g)}\right)}{\mathbf{f}_{F}}\right)\left(\frac{-1}{\mathbf{f}_{F}}\right)$ as in Subsection 4.2. We know that $x\left(\overline{\iota_{\mathcal{V}^{\prime}}(g)}\right)=$ $-(-1)^{2}(2 \varrho)^{-2}$. Therefore, we see that $m\left(\overline{\iota \mathcal{V}^{\prime}}(g)\right)=1$. Hence, $\alpha_{\mathcal{V}^{\prime}}(g)=$ 1.

8.2. . In this subsection, we assume that both $\mathcal{V}$ and $\mathcal{V}^{\prime}$ are two-dimensional and isotropic. Let $\left\{v_{1}, v_{2}\right\}$ (resp. $\left.\left\{v_{1}^{\prime}, v_{2}^{\prime}\right\}\right)$ be a standard basis of $\mathcal{V}\left(\right.$ resp. $\left.\mathcal{V}^{\prime}\right)$. Let $Y^{\circ}:=F v_{2}$ and $Y:=Y^{\circ} \otimes \mathcal{V}^{\prime}$.

Lemma. Suppose that both $\mathcal{V}$ and $\mathcal{V}^{\prime}$ are two-dimensional isotropic. Let $g:=\left[\begin{array}{cc}k & 0 \\ 0 & k^{-1}\end{array}\right]$ be an element in $U(\mathcal{V})$ with respect to the basis $\left\{v_{1}, v_{2}\right\}$ for some $k \in F^{\times}$such that $\operatorname{ord}(k)=0$. Then

$$
\begin{aligned}
& \beta_{\mathcal{V}^{\prime}}^{Y}(g)=1, \\
& \alpha_{\mathcal{V}^{\prime}}(g)=1 .
\end{aligned}
$$

Proof. From $[\mathbf{K l}]$ Theorem 3.1, we know that $\beta_{\mathcal{V}^{\prime}}^{Y}(g)=1$.

Let $w_{1}:=v_{1} \otimes v_{1}^{\prime}, w_{2}:=v_{1} \otimes v_{2}^{\prime}, w_{3}:=v_{2} \otimes v_{2}^{\prime}, w_{4}:=v_{2} \otimes v_{1}^{\prime}$. The set $\left\{w_{1}, w_{2}, w_{3}, w_{4}\right\}$ is a standard self-dual basis of $\mathcal{W}:=\mathcal{V} \otimes \mathcal{V}^{\prime}$. We have

$$
\iota_{\mathcal{V}^{\prime}}(g)=\left[\begin{array}{cccc}
k & 0 & 0 & 0 \\
0 & k & 0 & 0 \\
0 & 0 & k^{-1} & 0 \\
0 & 0 & 0 & k^{-1}
\end{array}\right] \in \operatorname{Sp}(\mathcal{W})
$$

with respect to the basis $\left\{w_{1}, w_{2}, w_{3}, w_{4}\right\}$. Then we know that the space $B^{*} / B$ is either trivial or four dimensional. In both case it is easy to see that $j\left(\overline{\left(\mathcal{V}^{\prime}(g)\right.}\right)=0$ and $x\left(\overline{\iota_{\mathcal{V}^{\prime}}(g)}\right)$ is a square. So $m\left(\overline{\left(\mathcal{V}^{\prime}(g)\right.}\right)=1$. Therefore, $\alpha_{\mathcal{V}^{\prime}}(g)=1$.

8.3. . In this subsection, we assume that both $\mathcal{V}$ and $\mathcal{V}^{\prime}$ are two-dimensional and isotropic. Let $\left\{v_{1}, v_{2}\right\}$ (resp. $\left\{v_{1}^{\prime}, v_{2}^{\prime}\right\}$ ) be a standard basis of $\mathcal{V}$ (resp. $\mathcal{V}^{\prime}$ ). Let $Y^{\circ}:=F v_{2}$ and $Y:=Y^{\circ} \otimes \mathcal{V}^{\prime}$.

Lemma. Suppose that both $\mathcal{V}$ and $\mathcal{V}^{\prime}$ are two-dimensional isotropic. Let $g:=\left[\begin{array}{cc}0 & k \\ k^{-1} & 0\end{array}\right]$ be an element in $U(\mathcal{V})$ with respect to the basis $\left\{v_{1}, v_{2}\right\}$ for some $k \in F^{\times}$such that $g \in U(\mathcal{V})_{L}$. Then

$$
\begin{aligned}
& \beta_{\mathcal{V}^{\prime}}^{Y}(g)=1, \\
& \alpha_{\mathcal{V}^{\prime}}(g)=1 .
\end{aligned}
$$

Proof. From [Kl] Theorem 3.1, we know that $\beta_{\mathcal{V}^{\prime}}^{Y}(g)=1$. 
Let $w_{1}:=v_{1} \otimes v_{1}^{\prime}, w_{2}:=v_{1} \otimes v_{2}^{\prime}, w_{3}:=v_{2} \otimes v_{2}^{\prime}, w_{4}:=v_{2} \otimes v_{1}^{\prime}$. The set $\left\{w_{1}, w_{2}, w_{3}, w_{4}\right\}$ is a standard self-dual basis of $\mathcal{W}:=\mathcal{V} \otimes \mathcal{V}^{\prime}$. We have

$$
\iota_{\mathcal{V}^{\prime}}(g)=\left[\begin{array}{cccc}
0 & 0 & 0 & k \\
0 & 0 & k & 0 \\
0 & k^{-1} & 0 & 0 \\
k^{-1} & 0 & 0 & 0
\end{array}\right] \in \operatorname{Sp}(\mathcal{W})
$$

with respect to the basis $\left\{w_{1}, w_{2}, w_{3}, w_{4}\right\}$. Let $L^{\prime}$ be a good lattice in $\mathcal{V}^{\prime}$. The space $B^{*} / B$ is either trivial or four-dimensional. In both case it is easy to see that $j\left(\overline{\iota \mathcal{V}^{\prime}(g)}\right)=2$ and $x\left(\overline{\left(\mathcal{V}^{\prime}(g)\right.}\right)=-\overline{k^{-2}}$. Hence by (1) in Subsection 1.5 and (4.2.a), we have $m\left(\overline{\iota \mathcal{V}^{\prime}(g)}\right)=\left(\frac{-\overline{\mathbf{f}^{-2}}}{\mathbf{f}_{F}}\right)\left(\frac{-1}{\mathbf{f}_{F}}\right)=1$. Therefore, $\alpha \mathcal{V}^{\prime}(g)=1$.

8.4. Proof of Proposition 3.3 for $\mathcal{V}$ quadratic. Let $\mathcal{V}$ be a quadratic space. So $D=F$ and both $U(\mathbf{l})$ and $U\left(\mathbf{l}^{*}\right)$ are finite orthogonal groups. In this case, we know that

$$
\begin{aligned}
S\left(U(\mathbf{l}) \times U\left(\mathbf{l}^{*}\right)\right) /\left(S U(\mathbf{l}) \times S U\left(\mathbf{l}^{*}\right)\right) \simeq \begin{cases}\{\operatorname{id}\}, & \text { if one of } \mathbf{l}, \mathbf{l}^{*} \text { is trivial; } \\
\mathbf{Z} / 2 \mathbf{Z}, & \text { if both } \mathbf{l}, \mathbf{l}^{*} \text { are not trivial, }\end{cases} \\
S U\left(\mathbf{l}^{\prime}\right) /\left[U\left(\mathbf{l}^{\prime}\right), U\left(\mathbf{l}^{\prime}\right)\right] \simeq \begin{cases}\mathbf{Z} / 2 \mathbf{Z}, & \text { if } \operatorname{dim}\left(\mathbf{l}^{\prime}\right) \geq 2 ; \\
\{\operatorname{id}\}, & \text { otherwise }\end{cases}
\end{aligned}
$$

where $\mathbf{l}^{\prime}$ is $\mathbf{l}$ or $\mathbf{l}^{*}$.

First, suppose that both $\mathbf{l}$ and $\mathbf{l}^{*}$ are nontrivial. Let $\mathcal{V}=\mathcal{V}_{1} \oplus \mathcal{V}_{2}$ be the decomposition in (1.3.c). For $i=1$ or 2 , if $\mathcal{V}_{i}$ is isotropic, choose an $L$-admissible hyperbolic plane $\mathcal{V}_{i}^{0}$ in $\mathcal{V}_{i}$ and let $g_{i}:=\left[\begin{array}{cc}0 & k_{i} \\ k_{i}^{-1} & 0\end{array}\right] \in U\left(\mathcal{V}_{i}^{0}\right) \subseteq$ $U(\mathcal{V})$ for some $k_{i} \in F^{\times}$. We can choose $k_{i}$ properly such that $g_{i} \in U(\mathcal{V})_{L}$. If $\mathcal{V}_{i}$ is anisotropic, choose an $L$-admissible nondegenerate one-dimensional subspace $\mathcal{V}_{i}^{0}$ and let $g_{i}:=-1 \in U\left(\mathcal{V}_{i}^{0}\right) \subseteq U(\mathcal{V})$. Therefore, $g_{i} \in U(\mathcal{V})_{L}$ and $g:=g_{1} g_{2}$ belongs to $S U(\mathcal{V})_{L}$. Moreover, the image of $g$ in $S(U(\mathbf{l}) \times$ $\left.U\left(\mathbf{l}^{*}\right)\right) /\left(S U(\mathbf{l}) \times S U\left(\mathbf{l}^{*}\right)\right)$ generates the group. We have an $L^{\prime}$-admissible decomposition $\mathcal{V}^{\prime}=\bigoplus_{i} \mathcal{V}_{i}^{\prime}$ of nondegenerate subspaces such that each $\mathcal{V}_{i}$ is a hyperbolic plane. Let $Y_{i}:=Y^{\circ} \otimes \mathcal{V}_{i}^{\prime}$. Then, we have

$$
\alpha_{\mathcal{V}^{\prime}}(g) \beta_{\mathcal{V}^{\prime}}^{Y}(g)=\prod_{i} \alpha_{\mathcal{V}_{i}^{\prime}}(g) \beta_{\mathcal{V}_{i}^{\prime}}^{Y_{i}}(g) .
$$

We have $\alpha_{\mathcal{V}_{i}^{\prime}}\left(g_{j}\right) \beta_{\mathcal{V}_{i}^{\prime}}^{Y_{i}}\left(g_{j}\right)=1$ for any $i, j$ by Lemmas 8.1 and 8.3. Since $\alpha_{\mathcal{V}_{i}^{\prime}} \beta_{\mathcal{V}_{i}^{\prime}}$ is a character of $U(\mathcal{V})_{L}$, we have $\alpha_{\mathcal{V}_{i}^{\prime}}(g) \beta_{\mathcal{V}_{i}}(g)=\alpha_{\mathcal{V}_{i}^{\prime}}\left(g_{1}\right) \beta_{\mathcal{V}_{i}}\left(g_{1}\right) \alpha_{\mathcal{V}_{i}^{\prime}}\left(g_{2}\right) \beta_{\mathcal{V}_{i}}\left(g_{2}\right)$ $=1$. Hence,

$$
\alpha \mathcal{V}^{\prime}(g) \beta_{\mathcal{V}^{\prime}}^{Y}(g)=1
$$


Next, suppose that the dimension of $\mathbf{l}$ is greater than 1 . We know that $\operatorname{dim}(\mathbf{l})=\operatorname{dim}\left(\mathcal{V}_{1}\right)$, so the dimension of $\mathcal{V}_{1}$ is greater than 1 . If $\mathcal{V}_{1}$ is isotropic, choose an $L$-admissible hyperbolic plane $\mathcal{V}_{i}^{0}$ in $\mathcal{V}_{i}$ and let $g:=$ $\left[\begin{array}{cc}k & 0 \\ 0 & k^{-1}\end{array}\right] \in U\left(\mathcal{V}_{i}^{0}\right) \subseteq U(\mathcal{V})$ for $k \in F^{\times}$such that $\operatorname{ord}(k)=0$ and $k$ is not a square. If $\mathcal{V}_{1}$ is anisotropic, then $\mathcal{V}_{1}$ is two-dimensional and has two $L$ admissible subspaces $F v_{1}, F v_{2}$ such that $\mathbf{h}\left(v_{1}, v_{1}\right) \mathbf{h}\left(v_{2}, v_{2}\right)$ is not a square. Let $g_{i}$ be the element such that $\left.g_{i}\right|_{F v_{i}}=-1$ and $\left.g_{i}\right|_{\left(F v_{i}\right)^{\perp}}=1$. Define $g:=g_{1} g_{2}$. Therefore, we know that $g$ belongs to $S U(\mathcal{V})_{L}$ and the image of $g$ in $S U(\mathcal{V})_{L} /\left[U(\mathcal{V})_{L}, U(\mathcal{V})_{L}\right]$ generates $S U(\mathbf{l}) /[U(\mathbf{l}), U(\mathbf{l})] \simeq \mathbf{Z} / 2 \mathbf{Z}$. Let $\mathcal{V}^{\prime}=\bigoplus_{i} \mathcal{V}_{i}^{\prime}$ be the decomposition as in the previous paragraph. If $\mathcal{V}_{1}$ is isotropic, then $\alpha_{\mathcal{V}_{i}^{\prime}}(g) \beta_{\mathcal{V}_{i}^{\prime}}(g)=1$ by Lemma 8.2. If $\mathcal{V}_{1}$ is anisotropic, then $\alpha_{\mathcal{V}_{i}^{\prime}}(g) \beta_{\mathcal{V}_{i}^{\prime}}(g)=\alpha_{\mathcal{V}_{i}^{\prime}}\left(g_{1}\right) \beta_{\mathcal{V}_{i}^{\prime}}\left(g_{1}\right) \alpha_{\mathcal{V}_{i}^{\prime}}\left(g_{2}\right) \beta_{\mathcal{V}_{i}^{\prime}}\left(g_{2}\right)=1$ by Lemma 8.1 . Hence, we obtain

$$
\alpha_{\mathcal{V}^{\prime}}(g) \beta_{\mathcal{V}^{\prime}}^{Y}(g)=\prod_{i} \alpha_{\mathcal{V}_{i}^{\prime}}(g) \beta_{\mathcal{V}_{i}^{\prime}}(g)=1
$$

If the dimension of $\mathbf{l}^{*}$ is greater than 1 , by exactly the same argument in the previous paragraph with $\mathbf{l}$ replaced by $\mathbf{l}^{*}$ and $\mathcal{V}_{1}$ replaced by $\mathcal{V}_{2}$ we can prove that

$$
\alpha_{\mathcal{V}^{\prime}}(g) \beta_{\mathcal{V}^{\prime}}^{Y}(g)=1
$$

for an element $g \in S U(\mathcal{V})_{L}$ such that the image of $g$ in $S U(\mathcal{V})_{L} /\left[U(\mathcal{V})_{L}\right.$, $\left.U(\mathcal{V})_{L}\right]$ generates $S U\left(\mathbf{l}^{*}\right) /\left[U\left(\mathbf{l}^{*}\right), U\left(\mathbf{l}^{*}\right)\right]$.

So by (8.4.b), (8.4.c) and (8.4.d) we conclude that $\alpha_{\mathcal{V}^{\prime}}(g) \beta_{\mathcal{V}^{\prime}}^{Y}(g)=1$ for any $g \in S U(\mathcal{V})_{L}$. Since $\zeta_{\mathcal{V}^{\prime}}$ is the trivial character of $U(\mathcal{V})_{L}$ is this case, we have $\left.\alpha_{\mathcal{V}^{\prime}} \beta_{\mathcal{V}^{\prime}}^{Y}\right|_{S U(\mathcal{V})_{L}}=\left.\zeta \mathcal{V}^{\prime}\right|_{S U(\mathcal{V})_{L}}$.

\section{Proof of Proposition 3.3 for symplectic groups.}

In this section, we assume that $\mathcal{V}$ is a symplectic space. In this case, $D=$ $F$ and both $U(\mathbf{l})$ and $U\left(\mathbf{l}^{*}\right)$ are finite symplectic groups. Therefore the quotient group $S\left(U(\mathbf{l}) \times U\left(\mathbf{l}^{*}\right)\right) /\left(S U(\mathbf{l}) \times S U\left(\mathbf{l}^{*}\right)\right)$ is trivial. The quotient $S U(\mathbf{l}) /[U(\mathbf{l}), U(\mathbf{l})]$ (resp. $S U\left(\mathbf{l}^{*}\right) /\left[U\left(\mathbf{l}^{*}\right), U\left(\mathbf{l}^{*}\right)\right]$ ) is nontrivial only if $q=3$ and the dimension of $\mathbf{l}$ (resp. $\mathbf{l}^{*}$ ) is two. As usual, fix a nontrivial character $\psi$ of $F$.

9.1. . In this subsection, we assume that $\mathcal{V}$ is two-dimensional and $\mathcal{V}^{\prime}$ is two-dimensional anisotropic. Let $\left\{v_{1}, v_{2}\right\}$ be a standard basis of $\mathcal{V}$. Let $Y^{\circ}:=F v_{2}$ and $Y:=Y^{\circ} \otimes \mathcal{V}^{\prime}$ 
Lemma. Suppose that $\mathcal{V}^{\prime}$ is two-dimensional anisotropic and $g:=\left[\begin{array}{cc}1 & \varpi_{F}^{d} \\ 0 & 1\end{array}\right]$ for some $d$ is an element of $U(\mathcal{V})_{L}$. Then,

$$
\begin{aligned}
& \beta_{\mathcal{V}^{\prime}}^{Y}(g)=1, \\
& \alpha_{\mathcal{V}^{\prime}}(g)=1 .
\end{aligned}
$$

Proof. It is clear that $j(g)=0$ and $x(g)=1$. By [Kl] Theorem 3.1, we know that

$$
\beta_{\mathcal{V}^{\prime}}^{Y}(g)=\left(x(g),-\operatorname{det}\left(\mathcal{V}^{\prime}\right)\right)_{F}=1 .
$$

Next, we want to compute $\alpha_{\mathcal{V}^{\prime}}(g)$. Because $\mathcal{V}^{\prime}$ is anisotropic, we have an $L$-admissible basis $\left\{v_{1}^{\prime}, v_{2}^{\prime}\right\}$ of $\mathcal{V}^{\prime}$ such that $\mathbf{h}^{\prime}\left(v_{1}^{\prime}, v_{2}^{\prime}\right)=0$. Let $\varrho_{1}:=\mathbf{h}^{\prime}\left(v_{1}^{\prime}, v_{1}^{\prime}\right)$ and $\varrho_{2}:=\mathbf{h}^{\prime}\left(v_{2}^{\prime}, v_{2}^{\prime}\right)$. We shall choose $v_{1}^{\prime}, v_{2}^{\prime}$ such that $\operatorname{ord}\left(\varrho_{1}\right), \operatorname{ord}\left(\varrho_{2}\right)$ are zero or one. Let $w_{1}:=\frac{1}{\varrho_{1}} v_{1} \otimes v_{1}^{\prime}, w_{2}:=\frac{1}{\varrho_{2}} v_{1} \otimes v_{2}^{\prime}, w_{3}:=v_{2} \otimes v_{1}^{\prime}, w_{4}:=$ $v_{2} \otimes v_{2}^{\prime}$. The set $\left\{w_{1}, w_{2}, w_{3}, w_{4}\right\}$ is a standard basis of $\mathcal{W}:=\mathcal{V} \otimes \mathcal{V}^{\prime}$. Then, $Y=F w_{3}+F w_{4}$. We have

$$
\iota_{\mathcal{V}^{\prime}}(g)=\left[\begin{array}{cccc}
1 & 0 & \varrho_{1} \varpi_{F}^{d} & 0 \\
0 & 1 & 0 & \varrho_{2} \varpi_{F}^{d} \\
0 & 0 & 1 & 0 \\
0 & 0 & 0 & 1
\end{array}\right] \in \operatorname{Sp}(\mathcal{W})
$$

with respect to the basis $\left\{w_{1}, w_{2}, w_{3}, w_{4}\right\}$. Let $L^{\prime}$ be a good lattice in $\mathcal{V}^{\prime}$. Therefore, the space $B^{*} / B$ might be trivial, two-dimensional or four dimensional.

(i) Suppose that $B^{*} / B$ is trivial. Hence, $m\left(\overline{\iota \mathcal{V}^{\prime}(g)}\right)=1$ and $j\left(\overline{\left(\mathcal{V}^{\prime}(g)\right.}\right)=0$. Therefore, $\alpha_{\mathcal{V}^{\prime}}(g)=1$.

(ii) Suppose that $B^{*} / B$ is two-dimensional. Then $\overline{\iota \mathcal{V}^{\prime}(g)}$ can be presented by a two by two upper triangular matrix with diagonal entries equal to 1 . Hence, $j\left(\overline{\iota \mathcal{V}^{\prime}(g)}\right)=0$ and $x\left(\overline{\iota \mathcal{V}^{\prime}(g)}\right)=1$. Therefore, $m\left(\overline{\iota \mathcal{V}^{\prime}(g)}\right)=1$ and $\alpha_{\mathcal{V}^{\prime}}(g)=1$.

(iii) Suppose that $B^{*} / B$ is four-dimensional. Then from (9.1.a) we see that $\overline{\iota^{\prime}(g)}$ can be presented as the $4 \times 4$ upper triangular matrix with diagonal entries equal to 1 . Hence, $j\left(\overline{\iota_{\mathcal{V}^{\prime}}(g)}\right)=0$ and $m\left(\overline{\iota_{\mathcal{V}^{\prime}}(g)}\right)=1$. Therefore, $\alpha_{\mathcal{V}^{\prime}}(g)=1$.

9.2. . In this subsection, we assume that both $\mathcal{V}$ and $\mathcal{V}^{\prime}$ are two-dimensional isotropic. Let $\left\{v_{1}, v_{2}\right\}$ be a standard basis of $\mathcal{V}$. Let $Y^{\circ}:=F v_{2}$ and $Y:=$ $Y^{\circ} \otimes \mathcal{V}^{\prime}$ 
Lemma. Suppose that $\mathcal{V}^{\prime}$ is two-dimensional isotropic and $g:=\left[\begin{array}{cc}1 & \varpi_{F}^{d} \\ 0 & 1\end{array}\right]$ for some $d$ is an element of $U(\mathcal{V})_{L}$. Then

$$
\begin{aligned}
& \beta_{\mathcal{V}^{\prime}}^{Y}(g)=1, \\
& \alpha_{\mathcal{V}^{\prime}}(g)=1 .
\end{aligned}
$$

Proof. As in Subsection 9.1, we know that $x(g)=1$ and $j(g)=0$. Therefore by $[\mathbf{K l}]$ Theorem 3.1, we have

$$
\beta_{\mathcal{V}^{\prime}}^{Y}(g)=\left(x(g), \operatorname{det}\left(\mathcal{V}^{\prime}\right)\right)_{F}=\left(1, \operatorname{det}\left(\mathcal{V}^{\prime}\right)\right)_{F}=1 .
$$

Now $\mathcal{V}^{\prime}$ is anisotropic, so $\mathcal{V}^{\prime}$ has a standard self-dual basis $\left\{v_{1}^{\prime}, v_{2}^{\prime}\right\}$ which is an $L$-admissible. Let $w_{1}:=v_{1} \otimes v_{1}^{\prime}, w_{2}:=v_{1} \otimes v_{2}^{\prime}, w_{3}:=v_{2} \otimes v_{2}^{\prime}, w_{4}:=v_{2} \otimes v_{1}^{\prime}$. The set $\left\{w_{1}, w_{2}, w_{3}, w_{4}\right\}$ is a standard self-dual basis of $\mathcal{W}:=\mathcal{V} \otimes \mathcal{V}^{\prime}$. Then

$$
\iota_{\mathcal{V}^{\prime}}(g)=\left[\begin{array}{cccc}
1 & 0 & 0 & \varpi_{F}^{d} \\
0 & 1 & \varpi_{F}^{d} & 0 \\
0 & 0 & 1 & 0 \\
0 & 0 & 0 & 1
\end{array}\right] \in \operatorname{Sp}(\mathcal{W})
$$

with respect to the basis $\left\{w_{1}, w_{2}, w_{3}, w_{4}\right\}$. Hence, $\iota \mathcal{V}^{\prime}(g)$ stabilizes $X=$ $F w_{1}+F w_{2}$. By the similar argument in Subsection 9.1, we can prove that $\alpha \mathcal{V}^{\prime}(g)=1$.

9.3. Proof of Proposition 3.3 for $\mathcal{V}$ symplectic. Now $\mathcal{V}$ is a symplectic space, we have

$S U(\mathcal{V})_{L} /\left[U(\mathcal{V})_{L}, U(\mathcal{V})_{L}\right] \simeq(S U(\mathbf{l}) /[U(\mathbf{l}), U(\mathbf{l})]) \times\left(S U\left(\mathbf{l}^{*}\right) /\left[U\left(\mathbf{l}^{*}\right), U\left(\mathbf{l}^{*}\right)\right]\right)$.

We know that $S U(\mathbf{l}) /[U(\mathbf{l}), U(\mathbf{l})]$ is nontrivial if and only if $\mathbf{l}$ is two-dimensional and $q=3$. (Note that we always assume that $q \neq 2$.)

First we assume that $\operatorname{dim}(\mathbf{l})=2$ and $q=3$. Let $\mathcal{V}=\mathcal{V}_{1} \oplus \mathcal{V}_{2}$ be the $L$-admissible decomposition as in (1.3.c). Therefore $\mathcal{V}_{1}$ is two-dimensional. Let $\left\{v_{1}, v_{2}\right\}$ be a standard basis of $\mathcal{V}_{1}$ and $g:=\left[\begin{array}{cc}1 & \varpi_{F}^{d} \\ 0 & 1\end{array}\right] \in U\left(\mathcal{V}_{1}\right) \subseteq U(\mathcal{V})$. We can choose the integer $d$ such that $g \in S U(\mathcal{V})_{L}=U(\mathcal{V})_{L}$ and the image of $g$ in $S U(\mathcal{V})_{L} /\left[U(\mathcal{V})_{L}, U(\mathcal{V})_{L}\right]$ generates $S U(\mathbf{l}) /[U(\mathbf{l}), U(\mathbf{l})]$. Because we assume that $\mathcal{V}^{\prime}$ is even-dimensional, we have an $L^{\prime}$-admissible decomposition $\mathcal{V}^{\prime}=\bigoplus_{i} \mathcal{V}_{i}^{\prime}$ such that each $\mathcal{V}_{i}^{\prime}$ is two-dimensional. We know that $\alpha_{\mathcal{V}_{i}^{\prime}}(g) \beta_{\mathcal{V}_{i}^{\prime}}^{Y_{i}}(g)=1$ for each $i$ by Lemmas 9.1 and 9.2. Hence,

$$
\alpha_{\mathcal{V}^{\prime}}(g) \beta_{\mathcal{V}^{\prime}}^{Y}(g)=\prod_{i} \alpha_{\mathcal{V}_{i}^{\prime}}(g) \beta_{\mathcal{V}_{i}^{\prime}}^{Y_{i}}(g)=1 .
$$

If $\mathbf{l}^{*}$ is two-dimensional, by the same argument we can prove that

$$
\alpha_{\mathcal{V}^{\prime}}(g) \beta_{\mathcal{V}^{\prime}}^{Y}(g)=1
$$


for $g \in S U(\mathcal{V})_{L}$ such that the image of $g$ in $S U(\mathcal{V})_{L} /\left[U(\mathcal{V})_{L}, U(\mathcal{V})_{L}\right]$ generates the group $S U\left(\mathbf{l}^{*}\right) /\left[U\left(\mathbf{l}^{*}\right), U\left(\mathbf{l}^{*}\right)\right]$.

Hence, we conclude that $\alpha_{\mathcal{V}^{\prime}}(g) \beta_{\mathcal{V}^{\prime}}^{Y}(g)=1$ for any $g \in S U(\mathcal{V})_{L}$. Since $\zeta_{\mathcal{V}^{\prime}}$ is trivial in this case, we get $\left.\alpha_{\mathcal{V}^{\prime}} \beta_{\mathcal{V}^{\prime}}^{Y}\right|_{S U(\mathcal{V})_{L}}=\left.\zeta \mathcal{V}^{\prime}\right|_{S U(\mathcal{V})_{L}}$.

\section{Splitting and theta dichotomy.}

In this section, we prove a proposition which concerns the splitting and theta dichotomy of unitary groups. Therefore, we assume that $D=E$ is a quadratic extension of $F$ throughout the section. The main result is Proposition 10.2, which will be a crucial ingredient of the results in [Pn2]. First we recall the notation from $[\mathbf{H K S}]$.

10.1. "Related" Witt towers. Denote the spaces $\mathcal{V}^{\prime+}, \mathcal{V}^{\prime-}$, so that

$$
\epsilon_{E / F}\left((-1)^{\frac{m^{ \pm}\left(m^{ \pm}-1\right)}{2}} \operatorname{det}\left(\mathcal{V}^{\prime \pm}\right)\right)= \pm 1
$$

where $m^{ \pm}$is the dimension of $\mathcal{V}^{\prime \pm}$. Let $\left\{\mathcal{V}_{m_{i}^{+}}^{+}\right\},\left\{\mathcal{V}_{m_{i}^{-}}^{-}\right\}$be two "related" Witt towers where $m_{i}^{+}$(resp. $m_{i}^{-}$) denotes the dimension of $\mathcal{V}_{m_{i}^{+}}^{+}\left(\right.$resp. $\mathcal{V}_{m_{i}^{-}}^{\prime-}$ ). Therefore, $\mathcal{V}_{m_{0}^{+}}^{+}$is trivial or a one-dimensional anisotropic $\epsilon^{\prime}$-hermitian space, $\mathcal{V}_{m_{0}^{-}}^{\prime-}$ is a one-dimensional or two-dimensional anisotropic $\epsilon^{\prime}$-hermitian space, and $\mathcal{V}_{m_{i}^{ \pm}}^{ \pm}$is the direct sum of $\mathcal{V}_{m_{0}^{ \pm}}^{ \pm}$and $i$ copies of hyperbolic planes; so $m_{i}^{ \pm}=m_{0}^{ \pm}+2 i$; and we know that the dimensions of $\left\{\mathcal{V}_{m_{i}^{+}}^{+}\right\},\left\{\mathcal{V}_{m_{i}^{-}}^{\prime-}\right\}$ are either all even or all odd.

If a character $\chi$ of $E^{\times}$such that $\left.\chi\right|_{F^{\times}}=\epsilon_{E / F}^{m^{ \pm}}$is fixed, then a splitting $U(\mathcal{V}) \rightarrow \widetilde{U(\mathcal{V})}$ is determined in $[\mathbf{K l}]$. Because $\epsilon_{E / F}$ is a character of order two, the character $\chi$ depends only on the parity of $m^{ \pm}$. Therefore we will fix $\chi$ such that $\left.\chi\right|_{F^{\times}}=\epsilon_{E / F}^{m_{0}^{ \pm}}$for the whole tower $\left\{\mathcal{V}_{m_{i}^{ \pm}}^{ \pm}\right\}$.

10.2. A character of the unitary group. Let $L$ be a good lattice in $\mathcal{V}$. We have a homomorphism $U(\mathcal{V})_{L} \rightarrow U(\mathbf{l}) \times U\left(\mathbf{l}^{*}\right)$. If $E$ is an unramified extension of $F$, then both $U(\mathbf{l}), U\left(\mathbf{l}^{*}\right)$ are finite unitary group. Let $\eta_{1}$ (resp. $\eta_{2}$ ) be the character of $U(\mathbf{l})$ (resp. $U\left(\mathbf{l}^{*}\right)$ ) of order two if the group is not trivial, and the trivial character if the group is trivial. If $E$ is a ramified extension of $F$, then one of $U(\mathbf{l}), U\left(\mathbf{l}^{*}\right)$ is an orthogonal group and the other is a symplectic group. Let $\eta_{1}$ (resp. $\eta_{2}$ ) be the character of order two whose restriction to $S U(\mathbf{l})$ (resp. $S U\left(\mathbf{l}^{*}\right)$ ) is trivial if the group is an orthogonal group, be the trivial character if the group is a symplectic group. For both cases let $\eta_{L}$ be the character of $U(\mathcal{V})_{L}$ lifted from $\eta_{1} \otimes \eta_{2}$ by the above homomorphism. 
It is easy to see that the character $\eta_{L}$ factors through the determinant map $\operatorname{det}: U(\mathcal{V})_{L} \rightarrow E^{(1)}$. Hence, $\eta_{L}$ is the restriction of a character $\eta$ of $U(\mathcal{V})$. In fact, $\eta$ is uniquely determined by $\eta_{L}$ except the cases that $E$ is a ramified extension of $F$ and one of $\mathbf{1}, \mathbf{l}^{*}$ is trivial and the trivial one is the quadratic space. For the exceptional case, $\eta$ could be the trivial character or the sgn character, otherwise $\eta$ is the sgn character of $U(\mathcal{V})$.

Proposition. Let $\eta_{L}$ be the character of $U(\mathcal{V})_{L}$ defined above. Let $\alpha_{\mathcal{V}^{ \pm}}$, $\beta_{\mathcal{V}^{\prime \pm}}^{Y^{ \pm}}$be the $\alpha_{\mathcal{V}^{\prime}}, \beta_{\mathcal{V}^{\prime}}^{Y}$ defined before for the dual pairs $\left(U(\mathcal{V}), U\left(\mathcal{V}^{\prime \pm}\right)\right)$. Then

$$
\alpha_{\mathcal{V}^{\prime+}}(g) \beta_{\mathcal{V}^{\prime+}}^{Y^{+}}(g)=\eta_{L}(g) \alpha_{\mathcal{V}^{\prime-}}(g) \beta_{\mathcal{V}^{\prime-}}^{Y^{-}}(g)
$$

for $g \in U(\mathcal{V})_{L}$.

From Proposition 3.3, we know that there exist characters $\zeta_{\mathcal{V}^{\prime}}$ and $\zeta_{\mathcal{V}^{\prime}}$ of $U(\mathcal{V})_{L}$ such that $\left.\alpha_{\mathcal{V}^{\prime} \pm} \beta_{\mathcal{V}^{\prime \pm}}^{Y^{ \pm}}\right|_{S U(\mathcal{V})_{L}}=\left.\zeta_{\mathcal{V}^{ \pm}}\right|_{S U(\mathcal{V})_{L}}$. We know that $\left.\eta_{L}\right|_{S U(\mathcal{V})_{L}}$ is trivial. We also know that $\zeta_{\mathcal{V}^{\prime}}$ depends only on the parity of the dimension of $\mathcal{V}^{\prime \pm}$. Therefore, $\zeta_{\mathcal{V}^{\prime}}(g)=\zeta_{\mathcal{V}^{\prime}}(g)$ for $g \in U(\mathcal{V})_{L}$. Hence, (10.2.a) is true when $g \in S U(\mathcal{V})_{L}$. So to prove the proposition, we need only to check (10.2.a) for $g$ runs over a set of representatives of $S U(\mathcal{V})_{L}$-cosets in $U(\mathcal{V})_{L}$. The remaining proof of the proposition will be in the next two subsections.

10.3. Proof of Proposition $\mathbf{1 0 . 2}$ for $E$ unramified. In this subsection, we assume that $E$ is an unramified quadratic extension of $F$. We know that $\mathcal{V}$ has an $L$-admissible decomposition $\mathcal{V}_{1} \oplus \mathcal{V}_{2}$ of nondegenerate subspaces such that $L \cap \mathcal{V}_{1}=L^{*} \cap \mathcal{V}_{1}$ and $L \cap \mathcal{V}_{2}=\varpi_{E}\left(L^{*} \cap \mathcal{V}_{2}\right)$. Without loss of generality, we may assume that $\mathcal{V}_{1}$ is not trivial. If $\mathcal{V}_{1}$ is isotropic, let $\mathcal{V}_{1}^{0}$ be an $L$-admissible hyperbolic plane in $\mathcal{V}_{1}$. If $\mathcal{V}_{1}$ is anisotropic, then $\mathcal{V}_{1}$ is one-dimensional. Let $\mathcal{V}_{1}^{0}:=\mathcal{V}_{1}$ for this case. Define a subgroup $H$ of $U\left(\mathcal{V}_{1}^{0}\right)$ by

$$
H:= \begin{cases}\left\{g \mid g:=\left[\begin{array}{cc}
\tau(k) & 0 \\
0 & k^{-1}
\end{array}\right], k \in E^{\times}, \operatorname{ord}(k)=0\right\}, & \text { if } \mathcal{V}_{1} \text { is isotropic; } \\
\left\{g \mid g:=t \in E^{(1)}\right\}, & \text { if } \mathcal{V}_{1} \text { is anisotropic. }\end{cases}
$$

It is clear that $H \subset U\left(\mathcal{V}_{1}^{0}\right)_{L_{1}} \subseteq U(\mathcal{V})_{L}$ where $L_{1}:=L \cap \mathcal{V}_{1}^{0}$. From the remark after Proposition 10.2, we only need to check that both $\alpha_{\mathcal{V}^{\prime+}} \beta_{\mathcal{V}^{\prime+}}^{Y^{+}}$ and $\eta_{L} \alpha_{\mathcal{V}^{\prime}} \beta_{\mathcal{V}^{\prime-}}^{Y^{-}}$agree on $H$. Now let $g$ be an element in $H$. Since the case for $g=1$ is always obvious, we assume that $g \neq 1$.

First, suppose that $\mathcal{V}_{1}$ is isotropic. If $\mathcal{V}^{\prime+}, \mathcal{V}^{\prime-}$ are both one-dimensional, we know that one of $\left(B^{+}\right)^{*} / B^{+},\left(B^{-}\right)^{*} / B^{-}$must be trivial and the other must be four-dimensional where $B^{ \pm}:=B\left(L_{1}, L^{\prime \pm}\right)$ and $L^{\prime \pm}$ is a good lattice in $\mathcal{V}^{ \pm}$. Therefore, by Lemma 6.4, we have

$$
\alpha_{\mathcal{V}^{\prime+}}(g) \beta_{\mathcal{V}^{\prime+}}^{Y^{+}}(g)=\left(\frac{\overline{k \tau(k)}}{\mathbf{f}_{F}}\right) \alpha_{\mathcal{V}^{\prime-}}(g) \beta_{\mathcal{V}^{\prime-}}^{Y^{-}}(g) .
$$


Clearly the map $g \mapsto\left(\frac{\overline{k \tau(k)}}{\mathbf{f}_{F}}\right)$ is the character $\left.\eta_{L}\right|_{H}$. If both $\mathcal{V}^{\prime+}, \mathcal{V}^{\prime-}$ are two-dimensional, then $\mathcal{V}^{\prime+}$ is isotropic and $\mathcal{V}^{\prime-}$ is anisotropic. Therefore, $\alpha_{\mathcal{V}^{\prime}} \beta_{\mathcal{V}^{\prime}}^{Y^{-}}(g)=\left(\frac{\overline{k \tau(k)}}{\mathbf{f}_{F}}\right) \chi(\tau(k))$ by Lemma 6.6. On the other hand, we have $\alpha_{\mathcal{V}^{\prime}+} \beta_{\mathcal{V}^{\prime+}}^{Y^{+}}(g)=\chi(\tau(k))$ by Lemma 6.5 . Hence, the lemma is proved in this case.

Next, suppose that $\mathcal{V}_{1}$ is anisotropic. If $\mathcal{V}^{\prime+}, \mathcal{V}^{\prime-}$ are both one-dimensional, we know that one of ord $\left(\varrho^{+}\right)$and $\operatorname{ord}\left(\varrho^{-}\right)$must be even and the other must be odd where $\varrho^{ \pm}$is the $\varrho$ in Subsection 6.1. From Lemma 6.1, we get

$$
\alpha_{\mathcal{V}^{\prime}}(g) \beta_{\mathcal{V}^{\prime+}}^{Y^{+}}(g)= \begin{cases}-\left(\frac{\overline{2 x-2}}{\mathbf{f}_{F}}\right) \alpha_{\mathcal{V}^{\prime}}(g) \beta_{\mathcal{V}^{\prime-}}^{Y^{-}}(g), & \text { if } x \neq 1 \bmod \mathfrak{p}_{F} ; \\ \alpha_{\mathcal{V}^{\prime}}(g) \beta_{\mathcal{V}^{\prime}}^{Y^{-}}(g) & \text { if } x \equiv 1 \bmod \mathfrak{p}_{F} .\end{cases}
$$

It is straightforward to see that the map $g \mapsto-\left(\frac{\overline{2 x-2}}{\mathbf{f}_{F}}\right)$ if $x \not \equiv 1 \bmod \mathfrak{p}_{F}$ and $g \mapsto 1$ if $x \equiv 1 \bmod \mathfrak{p}_{F}$ is exactly the character $\left.\eta_{L}\right|_{H}$. If $\mathcal{V}^{\prime \pm}$ are two-dimensional, then $\mathcal{V}^{\prime+}$ is isotropic and $\mathcal{V}^{\prime-}$ is anisotropic. Therefore,

$$
\alpha_{\mathcal{V}^{\prime}}(g) \beta_{\mathcal{V}^{\prime}}^{Y^{-}}(g)= \begin{cases}-\left(\frac{\overline{2 x-2}}{\mathbf{f}_{F}}\right) \chi((t-1) \delta), & \text { if } x \neq 1 \bmod \mathfrak{p}_{F} \\ \chi((t-1) \delta), & \text { if } x \equiv 1 \bmod \mathfrak{p}_{F}\end{cases}
$$

by Lemma 6.3. On the other hand, we have $\alpha_{\mathcal{V}^{\prime}}(g) \beta_{\mathcal{V}^{\prime+}}^{Y^{+}}(g)=\chi((t-1) \delta)$ by Lemma 6.2. Therefore, the proposition is proved in this case.

So we have proved the proposition when $\mathcal{V}^{\prime+}, \mathcal{V}^{\prime-}$ are both one-dimensional or both two-dimensional. Now we want to consider the general situation. We have an $L^{\prime}$-admissible orthogonal decomposition

$$
\mathcal{V}^{\prime \pm}=\mathcal{V}_{0}^{\prime \pm} \oplus \bigoplus_{i=1}^{l^{ \pm}} \mathcal{V}_{i}^{\prime+}
$$

such that each $\mathcal{V}_{i}^{\prime+}$ is two-dimensional isotropic for $i \geq 1$ and either both $\mathcal{V}_{0}^{\prime \pm}$ are one-dimensional, or $\mathcal{V}_{0}^{\prime+}$ is two-dimensional isotropic and $\mathcal{V}_{0}^{\prime-}$ is twodimensional anisotropic. Since $\epsilon_{E / F}^{m_{i}}$ is trivial for $i \geq 1$. Let $\chi_{i}$ be characters of $E^{\times}$with respect to the decomposition (10.3.a) as in Subsection 3.6. We choose $\chi_{i}$ to be trivial for $i \geq 1$ and $\chi_{0}:=\chi$. Therefore, $\chi=\bigotimes_{i} \chi_{i}$. Thus,

$$
\alpha_{\mathcal{V}^{\prime \pm}}(g) \beta_{\mathcal{V}^{\prime \pm}}^{Y^{ \pm}}(g)=\alpha_{\mathcal{V}_{0}^{\prime \pm}}(g) \beta_{\mathcal{V}_{0}^{\prime \pm}}^{Y_{0}^{ \pm}}(g) \prod_{i=1}^{l^{ \pm}} \alpha_{\mathcal{V}_{i}^{\prime \pm}}(g) \beta_{\mathcal{V}_{i}^{\prime \pm}}^{Y_{i}^{ \pm}}(g)
$$

as in Subsection 3.6. We know that each $\alpha_{\mathcal{V}_{i}^{\prime \pm}}(g) \beta_{\mathcal{V}_{i}^{\prime \pm}}^{Y^{ \pm}}(g)$ is equal to 1 for $i \geq 1$ by Lemma 6.2 and 6.5 because $\chi_{i}$ is trivial, and $\alpha_{\mathcal{V}_{0}^{\prime+}}(g) \beta_{\mathcal{V}_{0}^{\prime+}}^{Y^{+}}(g)=$ $\eta_{L}(g) \alpha_{\mathcal{V}_{0}^{\prime-}}(g) \beta_{\mathcal{V}_{0}^{\prime-}}^{Y_{-}^{-}}(g)$ as proved in the previous two paragraphs. Therefore, 
Proposition 10.2 is proved when $\mathcal{V}$ is over an unramified quadratic extension of $F$.

10.4. Proof of Proposition $\mathbf{1 0 . 2}$ for $E$ ramified. Let $\mathcal{V}=\mathcal{V}_{1} \oplus \mathcal{V}_{2}$ be the decomposition as in Subsection 10.3. Let $L_{i}:=L \cap \mathcal{V}_{i}$. It is clear that $L_{i}$ is a good lattice in $\mathcal{V}_{i}$ and we have homomorphisms

$$
U\left(\mathcal{V}_{1}\right)_{L_{1}} \times U\left(\mathcal{V}_{2}\right)_{L_{2}} \longrightarrow U(\mathcal{V})_{L} \longrightarrow U(\mathbf{l}) \times U\left(\mathbf{l}^{*}\right)
$$

Moreover the composition gives rise surjective homomorphisms $U\left(\mathcal{V}_{1}\right)_{L_{1}} \rightarrow$ $U(\mathbf{l})$ and $U\left(\mathcal{V}_{2}\right)_{L_{2}} \rightarrow U\left(\mathbf{l}^{*}\right)$. Now we know that one of $\mathbf{l}, \mathbf{l}^{*}$ is an orthogonal space and the other is a symplectic space. Without loss of generality, we may assume that $\mathbf{l}$ is an orthogonal space and $\mathbf{l}^{*}$ is a symplectic space. Therefore, $\mathcal{V}_{2}$ is always isotropic. Of course, we assume that $\mathcal{V}$ is not trivial. Define a subgroup $H$ of $U(\mathcal{V})_{L}$ as follows. If $\mathcal{V}_{1}$ is nontrivial and isotropic, let $\mathcal{V}_{1}^{0}$ be an $L$-admissible hyperbolic plane in $\mathcal{V}_{1}$ and define

$$
\begin{aligned}
H:=\left\{g_{1}, g_{2} \mid g_{1}:=\left[\begin{array}{cc}
\tau(k) & 0 \\
0 & k^{-1}
\end{array}\right], g_{2}:=\left[\begin{array}{cc}
0 & \epsilon k^{\prime-1} \\
\tau\left(k^{\prime}\right) & 0
\end{array}\right],\right. \\
\left.k, k^{\prime} \in E^{\times}, \operatorname{ord}(k)=0, \operatorname{ord}\left(k^{\prime}\right)=s\right\}
\end{aligned}
$$

for some integer $s$ such that $H \subset U(\mathcal{V})_{L}$. If $\mathcal{V}_{1}$ is nontrivial and anisotropic, let $\mathcal{V}_{1}^{0}$ be an $L$-admissible nondegenerate one-dimensional subspace and define $H:=U\left(\mathcal{V}_{1}^{0}\right) \subseteq U(\mathcal{V})_{L}$. In this case, we will identify $H$ with $E^{(1)}$ by a choice of a basis of $\mathcal{V}_{1}^{0}$. If $\mathcal{V}_{1}$ is trivial, let $\mathcal{V}_{2}^{0}$ be an $L$-admissible hyperbolic plane in $\mathcal{V}_{2}$ and define

$$
H:=\left\{g \mid g:=\left[\begin{array}{cc}
\tau(k) & 0 \\
0 & k^{-1}
\end{array}\right], k \in E^{\times}, \operatorname{ord}(k)=0\right\} \subseteq U(\mathcal{V})_{L} .
$$

It is straightforward to check that the map $\left.\operatorname{det}\right|_{H}$ is still surjective to $E^{(1)}$ if $\mathcal{V}_{1}$ is not trivial. If $\mathcal{V}_{1}$ is trivial, then $\operatorname{det}(H)$ is a subgroup of $E^{(1)}$ of index two. Let $g$ be an element of $H$ and $g \neq 1$.

First, suppose that $\mathcal{V}_{1}$ is isotropic. Consider the dual pairs $\left(U\left(\mathcal{V}_{1}^{0}\right)\right.$, $\left.U\left(\mathcal{V}^{\prime \pm}\right)\right)$. If $\mathcal{V}^{\prime+}, \mathcal{V}^{\prime-}$ are both one-dimensional, it is clear that both $\left(B^{+}\right)^{*} / B^{+}$ and $\left(B^{-}\right)^{*} / B^{-}$are trivial where $B^{ \pm}$are defined as in Subsection 10.3. Let $\mathbf{h}^{\prime \pm}$ denote the form on $\mathcal{V}^{\prime \pm}$. Choose a nonzero element $v^{\prime \pm} \in \mathcal{V}^{\prime \pm}$. Define $r^{\prime \pm}:=\mathbf{h}^{\prime \pm}\left(v^{\prime \pm}, v^{\prime \pm}\right)$ if $\epsilon^{\prime}=1$ and $r^{\prime \pm}:=\delta \mathbf{h}^{\prime \pm}\left(v^{\prime \pm}, v^{\prime \pm}\right)$ if $\epsilon^{\prime}=-1$. It is clear 
that $\left(\Delta,-r^{\prime+} r^{-}\right)_{F}=-1$. By Lemma 7.4, we have

$$
\begin{aligned}
& \alpha_{\mathcal{V}^{ \pm}}(g) \beta_{\mathcal{V}^{ \pm}}^{Y^{ \pm}}(g) \\
& = \begin{cases}\chi(\tau(k)), & \text { if } g=g_{1} ; \\
\chi(\tau(k))\left(\Delta,-r^{ \pm}\right)_{F} \gamma_{\mathbf{f}_{F}}\left(\bar{\psi}_{0}\right)^{-1}\left(\frac{-1}{\mathbf{f}_{F}}\right)^{\overline{\operatorname{ord}}\left(\psi_{0}\right)}, & \text { if } g=g_{2} \text { and } \epsilon=-1 ; \\
\chi(\tau(k) \delta)\left(\Delta, r^{\prime \pm}\right)_{F} \gamma_{\mathbf{f}_{F}}\left(\bar{\psi}_{0}\right)^{-1}\left(\frac{-1}{\mathbf{f}_{F}}\right)^{\overline{\operatorname{ord}}\left(\psi_{0}\right)}, & \text { if } g=g_{2} \text { and } \epsilon=1 .\end{cases}
\end{aligned}
$$

Therefore, we have

$$
\alpha_{\mathcal{V}^{\prime+}}(g) \beta_{\mathcal{V}^{\prime+}}^{Y^{ \pm}}(g)=\left\{\begin{aligned}
\alpha_{\mathcal{V}^{-}}(g) \beta_{\mathcal{V}^{\prime}}^{Y^{-}}(g), & \text { if } g=g_{1} \\
-\alpha_{\mathcal{V}^{\prime}}(g) \beta_{\mathcal{V}^{\prime}}^{Y^{-}}(g), & \text { if } g=g_{2} .
\end{aligned}\right.
$$

Note that $\operatorname{det}\left(g_{1}\right) \equiv 1 \bmod \mathfrak{p}_{E}$ and $\operatorname{det}\left(g_{2}\right) \equiv-1 \bmod \mathfrak{p}_{E}$. Hence, the map $g_{1} \mapsto 1$ and $g_{2} \mapsto-1$ is exactly the character $\left.\eta_{L}\right|_{H}$. If $\mathcal{V}^{\prime \pm}$ are twodimensional, $\mathcal{V}^{\prime+}$ is isotropic and $\mathcal{V}^{-}$is anisotropic. We should notice that $\left(B^{-}\right)^{*} / B^{-}$is trivial. Therefore, by Lemma 7.6 , we have

$$
\alpha_{\mathcal{V}^{\prime}}(g) \beta_{\mathcal{V}^{\prime}}^{Y^{-}}(g)=\left\{\begin{aligned}
\chi(\tau(k)), & \text { if } g=g_{1} ; \\
-\chi\left(\tau\left(k^{\prime}\right)\right), & \text { if } g=g_{2} \text { and } \epsilon=-1 \\
-\chi\left(\tau\left(k^{\prime}\right) \delta\right), & \text { if } g=g_{2} \text { and } \epsilon=1 .
\end{aligned}\right.
$$

On the other hand, we have

$$
\alpha_{\mathcal{V}^{\prime}} \beta_{\mathcal{V}^{\prime+}}^{Y^{+}}(g)= \begin{cases}\chi(\tau(k)), & \text { if } g=g_{1} ; \\ \chi\left(\tau\left(k^{\prime}\right)\right), & \text { if } g=g_{2} \text { and } \epsilon=-1 \\ \chi\left(\tau\left(k^{\prime}\right) \delta\right), & \text { if } g=g_{2} \text { and } \epsilon=1\end{cases}
$$

by Lemma 7.5. Hence, the lemma is proved in this case.

Suppose that $\mathcal{V}_{1}$ is anisotropic. Write $g=t$ for some $t \in E^{(1)}$. If $\mathcal{V}^{ \pm \pm}$are one-dimensional, from Lemma 7.1 we know that (10.4.b)

$$
\alpha_{\mathcal{V}^{\prime \pm}}(g) \beta_{\mathcal{V}^{\prime \pm}}^{Y^{ \pm}}(g)= \begin{cases}\chi((t-1) \delta)\left(\Delta, \varrho^{ \pm}\right)_{F} \gamma_{F}\left(\Delta, \psi_{0}\right)^{-1}, & \text { if } x \not \equiv 1 \bmod \mathfrak{p}_{F} \\ \chi((t-1) \delta)(\Delta,-2 y)_{F}, & \text { if } x \equiv 1 \bmod \mathfrak{p}_{F}\end{cases}
$$

where $\varrho^{ \pm}$are defined analogously as Subsection 10.3. We know that $\left(\Delta, \varrho^{+}\right)_{F},\left(\Delta, \varrho^{-}\right)_{F}$ is 1 and the other is -1 , so we have

$$
\alpha_{\mathcal{V}^{\prime}}(g) \beta_{\mathcal{V}^{\prime+}}^{Y^{+}}(g)=\left\{\begin{array}{cc}
-\alpha_{\mathcal{V}^{\prime}}(g) \beta_{\mathcal{V}^{\prime}}^{Y^{-}}(g), & \text { if } x \neq 1 \bmod \mathfrak{p}_{F} \\
\alpha_{\mathcal{V}^{\prime}}(g) \beta_{\mathcal{V}^{-}}^{Y^{-}}(g) & \text { if } x \equiv 1 \bmod \mathfrak{p}_{F} .
\end{array}\right.
$$


It is obvious that the map $g \mapsto-1$ if $x \not \equiv 1 \bmod \mathfrak{p}_{F}$ and $g \mapsto 1$ if $x \equiv 1 \bmod \mathfrak{p}_{F}$ is exactly the character $\left.\eta_{L}\right|_{H}$. Suppose that $\mathcal{V}^{\prime \pm}$ are twodimensional. By Lemma 7.3, we have

$$
\alpha_{\mathcal{V}^{\prime}}(g) \beta_{\mathcal{V}^{\prime}}^{Y^{-}}(g)=\left\{\begin{aligned}
-\chi((t-1) \delta), & \text { if } x \neq 1 \bmod \mathfrak{p}_{F} \\
\chi((t-1) \delta), & \text { if } x \equiv 1 \bmod \mathfrak{p}_{F} .
\end{aligned}\right.
$$

On the other hand, we have

$$
\alpha_{\mathcal{V}^{\prime+}}(g) \beta_{\mathcal{V}^{\prime+}}^{Y^{+}}(g)=\chi((t-1) \delta)
$$

from Lemma 7.2. Therefore, the proposition is proved in this case.

Suppose that $\mathcal{V}_{1}$ is trivial. Hence, $g=\left[\begin{array}{cc}\tau(k) & 0 \\ 0 & k^{-1}\end{array}\right]$ for some $k$. Consider the dual pairs $\left(U\left(\mathcal{V}_{2}^{0}\right), U\left(\mathcal{V}^{ \pm}\right)\right)$. If $\mathcal{V}^{\prime+}, \mathcal{V}^{-}$are both one-dimensional, then

$$
\alpha_{\mathcal{V}^{\prime \pm}}(g) \beta_{\mathcal{V}^{\prime \pm}}^{Y^{ \pm}}(g)= \begin{cases}\chi(\tau(k)), & \text { if }\left(B^{ \pm}\right)^{*} / B^{ \pm} \text {is trivial; } \\ \left(\frac{\bar{x}_{0}}{\mathbf{f}_{F}}\right) \chi(\tau(k)), & \text { if }\left(B^{ \pm}\right)^{*} / B^{ \pm} \text {is not trivial }\end{cases}
$$

by Lemma 7.4. Hence,

$$
\alpha_{\mathcal{V}^{\prime+}}(g) \beta_{\mathcal{V}^{\prime+}}^{Y^{+}}(g)=\alpha_{\mathcal{V}^{\prime}}(g) \beta_{\mathcal{V}^{\prime-}}^{Y^{-}}(g) .
$$

If $\mathcal{V}^{\prime+}, \mathcal{V}^{\prime-}$ are both two-dimensional, then we see that

$$
\alpha_{\mathcal{V}^{\prime}}(g) \beta_{\mathcal{V}^{\prime-}}^{Y^{-}}(g)=\chi(\tau(k))=\alpha_{\mathcal{V}^{\prime+}}(g) \beta_{\mathcal{V}^{\prime+}}^{Y^{+}}(g)
$$

by Lemmas 7.5 and 7.6. We know that $\left.\eta_{L}\right|_{H}$ is trivial in this case. So the proposition is proved in this case.

The general situation follows the previous three cases by the similar argument in Subsection 10.3.

\section{References}

[BT] F. Bruhat and J. Tits, Schémas en groupes et immeubles des groupes classiques sur un corps local, deuxième partie: Groupes unitaires, Bull. Soc. Math. France, 115 (1987), 141-195.

[HO] A. Hahn and O.T. O'Meara, The Classical Groups and K-Theory, SpringerVerlag, Berlin-Heidelberg-New York, 1989.

[HKS] M. Harris, S. Kudla and W. Sweet, Theta dichotomy for unitary groups, J. Amer. Math. Soc., 9 (1996), 941-1004.

[Hj] H. Hijikata, Maximal Compact Subgroups of Some p-Adic Classical Groups, mimeographed notes, Yale University, 1964.

[Kl] S. Kudla, Splitting metaplectic covers of dual reductive pairs, Israel J. Math., 87 (1994), 361-401.

[Md] D. Manderscheid, Supercuspidal representations and the theta correspondence, J. Algebra, 151 (1992), 375-407. 
[MVW] C. Møglin, M.-F. Vigneras and J.-L. Waldspurger, Correspondances de Howe sur un Corps p-Adiques, Lecture Notes in Math., 1291, Springer-Verlag, BerlinHeidelberg-New York, 1987.

[Mn] C. Moen, The dual pair $(U(1), U(1))$ over a p-adic field, Pacific J. Math., 158 (1993), 365-386.

[Pn1] S.-Y. Pan, The commutator subgroups of maximal open compact subgroups of padic classical groups, submitted, 1999.

[Pn2] Local theta correspondence of depth zero representations and theta dichotomy, preprint, 1999.

[Pn3] - Local theta correspondence and unrefined minimal K-types, submitted, 1999.

[RR] R. Ranga-Rao, On some explicit formulas in the theory of Weil representation, Pacific J. Math., 157 (1993), 335-371.

[Wp] J.-L. Waldspurger, Demonstration d'une conjecture de duality de Howe dans le case $p$-adiques, $p \neq 2$, in 'Festschrift in honor of I. Piatetski-Shapiro', Israel Math. Conf. Proc., 2 (1990), 267-324.

[Yn] T. Yang, Eigenfunctions of the Weil representation of unitary group of one variable, Trans. Amer. Math. Soc., 350 (1998), 2393-2407.

Received April 29, 1999 and revised March 21, 2000.

National Cheng Kung University

TAINAN City 701

TAIWAN

E-mail address: sypan@mail.ncku.edu.tw 